\title{
NLO BFKL equation, running coupling, and renormalization scales
}

\author{
Robert S. Thorne \\ Jesus College and Theoretical Physics, University of Oxford, Oxford, Oxon OX1 3DW, United Kingdom
}

(Received 22 January 1999; published 12 August 1999)

\begin{abstract}
I examine the solution of the BFKL equation with NLO corrections relevant for deep inelastic scattering. Particular emphasis is placed on the part played by the running of the coupling. It is shown that the solution factorizes into a part describing the evolution in $Q^{2}$, and a constant part describing the input distribution. The latter is infrared dominated, being described by a coupling which grows as $x$ decreases, and thus being contaminated by infrared renormalons. Hence, for this part we agree with previous assertions that predictive power breaks down for small enough $x$ at any $Q^{2}$. However, the former is ultraviolet dominated, being described by a coupling which falls like $1 /\left(\ln \left(Q^{2} / \Lambda^{2}\right)+A\left[\bar{\alpha}_{s}\left(Q^{2}\right) \ln (1 / x)\right]^{1 / 2}\right)$ with decreasing $x$, and thus is perturbatively calculable at all $x$. Therefore, although the BFKL equation is unable to predict the input for a structure function for small $x$, it is able to predict its evolution in $Q^{2}$, as we would expect from the factorization theory. The evolution at small $x$ has no true powerlike behavior due to the fall of the coupling, but does have significant differences from that predicted from a standard NLO in $\alpha_{s}$ treatment. Application of the resummed splitting functions with the appropriate coupling constant to an analysis of data, i.e., a global fit, is very successful. [S0556-2821(99)07213-6]
\end{abstract}

PACS number(s): 13.60.Hb, 12.38.Bx

\section{INTRODUCTION}

There has recently been a great deal of interest in the solution to the Balitskiı̌-Fadin-Kuraev-Lipatov (BFKL) equation [1], triggered by the calculation of the next leading order (NLO) correction to the kernel [2,3] and the apparent result that this leads to a huge correction to the LO result. A number of subsequent papers have examined the solutions to this equation and/or its consequences [4-9] drawing a variety of conclusions (dominant negative NLO anomalous dimensions, oscillatory behavior, non-Regge terms, instability, breakdown of perturbation theory), most being rather pessimistic. This has prompted work on ways to at least estimate contributions to the kernel at even higher orders, and obtain perturbative stability via a summation $[10,11]$.

I will take the point of view that the most significant result of the NLO kernel is that it indicates very strongly how the coupling constant should run in the BFKL equation, i.e., that the scale in the coupling should be chosen to be the transverse momentum at the top of the gluon ladder $k^{2}$. Making this choice [12] I follow many previous authors in examining how this choice affects the solution to the LO equation [13-21]. I find that at leading twist the solution factorizes into a part dependent of the input to the equation, but independent of the scale $k^{2}$, and a part independent of the input which governs the evolution in $k^{2}[14,15,21]$. The former is disastrously contaminated by the diffusion [22] into the infrared, and without a low $k^{2}$ regularization is indeterminate due to the presence of infrared renormalons giving behavior $\sim \exp \left(-n \beta_{0}\left[\ln \left(Q_{0}^{2} / \Lambda^{2}\right)\right]^{3} /\left[A^{2} \ln (1 / x)\right]\right)$, where $Q_{0}^{2}$ is the scale of the input to the equation, $n$ is an integer, and $A$ $\sim 4$. This is entirely consistent with Mueller's result $[23,7]$ on the range of applicability of the BFKL equation. It renders the NLO correction to the kernel which is not associated with running of the coupling rather unimportant since the infrared contamination renders even the LO result untrustworthy.
However, the part of the solution governing the evolution in $k^{2}$ is not only infrared safe but is influenced strongly by diffusion into the ultraviolet. Hence the effective scale in the problem is greater than $k^{2}$, and this increase becomes more significant as $x$ decreases. This leads to the effective coupling constant decreasing as we go to smaller $x$, behaving like $1 /\left(\left(\ln k^{2} / \Lambda^{2}\right)+A\left[\ln (1 / x) / \ln \left(k^{2} / \Lambda^{2}\right)\right]^{1 / 2}\right) \quad$ rather than $1 /\left(\ln \left(k^{2} / \Lambda^{2}\right)\right)$. This result is quantified by using the BLM scale fixing procedure [24] for both LO and NLO quantities, obtaining precisely the same result of $A=3.63$ in both cases. It suggests that the effective splitting function governing the evolution does not grow like a power of $x^{-1-\lambda}$ as $x \rightarrow 0$, but is softened to something of the form $(1 / x) \exp \left([\ln (1 / x)]^{1 / 2} \rho\left(k^{2}\right)\right)$, though it seems difficult to obtain the precise form. This result means that the NLO corrections to the kernel not concerned with the running of the coupling are also relatively unimportant for the term governing the evolution, simply because the coupling constant associated with them is so small. Therefore, it seems as though we have good predictive power for the evolution of the gluon at small $x$, but that it is very different from the LO-BFKL prediction with fixed $\alpha_{s}$. Because the behavior of physical structure functions at small $x$ is related to the gluon via the convolution of a $k^{2}$-dependent cross section at the top of the gluon ladder [25,26], all such effects are associated with the ultraviolet diffusion. Hence, the evolution of physical quantities is governed by the same effective coupling constant, and is completely predictive, being somewhat different from both the LO-BFKL predictions with fixed $\alpha_{s}$ and the fixed order in $\alpha_{s}\left(Q^{2}\right)$ Dokshitzer-Gribov-Lipatov-Altarelli-Parisi (DGLAP) descriptions.

In this paper I will demonstrate the results discussed above. I will start with a brief discussion of the LO BFKL solution with fixed coupling, emphasising the role played by the infrared and ultraviolet regions of transverse momentum. I will then look at the same equation for running coupling, showing how the solutions change. This will facilitate a dis- 
cussion of the real importance of the total NLO correction to the BFKL equation. Finally, I will examine the implications of my results for physical quantities and give a brief outline of phenomenological consequences, showing that my results work very well when used to analyze experimental data. I note that a very brief account of this work, which nevertheless contains many of the main ideas, appears in [27].

\section{BFKL EQUATION FOR FIXED $\alpha_{s}$}

The BFKL equation for zero momentum transfer is an integral equation for the 4-point $k_{T}$-dependent gluon Green's function for forward scattering in the high energy limit, $f\left(k_{1}, k_{2}, \alpha_{s} / N\right)$ where $N$ is the Mellin conjugate variable to energy. Throughout this paper I will consider the canonical physical process of deep-inelastic scattering where the bottom leg is convoluted with a bare gluon density and the top leg with an off-shell hard scattering process. Hence, $k_{2}$ is taken to be some fixed scale $Q_{0}^{2}$ typical of soft physics, ${ }^{1}$ while $k_{1}^{2}=k^{2}$, i.e., a variable scale typically $\gg Q_{0}^{2}$. In this case $N$ is the conjugate variable to $x$, i.e., we define the moment space structure functions by the Mellin transformation,

$$
\mathcal{F}\left(N, Q^{2}\right)=\int_{0}^{1} x^{N-1} F\left(x, Q^{2}\right) d x,
$$

and the moment space parton distributions as the Mellin transformation of a rescaled parton density, i.e.,

$$
f\left(N, Q^{2}\right)=\int_{0}^{1} x^{N} f\left(x, Q^{2}\right) d x
$$

Using these definitions the BFKL equation becomes

$$
f\left(k^{2}, Q_{0}^{2}, \bar{\alpha}_{s} / N\right)=f^{0}\left(k^{2}, Q_{0}^{2}\right)+\frac{\bar{\alpha}_{s}}{N} \int_{0}^{\infty} \frac{d q^{2}}{q^{2}} K^{0}\left(q^{2}, k^{2}\right) f\left(q^{2}\right),
$$

where

$$
K^{0}\left(q^{2}, k^{2}\right) f\left(q^{2}\right)=k^{2}\left(\frac{f\left(q^{2}\right)-f\left(k^{2}\right)}{\left|k^{2}-q^{2}\right|}+\frac{f\left(k^{2}\right)}{\left(4 q^{4}+k^{4}\right)^{1 / 2}}\right),
$$

$f^{0}\left(k^{2}, Q_{0}^{2}\right)$ is the zeroth order input, and $\bar{\alpha}_{s}=(3 / \pi) \alpha_{s}$. As a simple choice I take

$$
f^{0}\left(k^{2}, Q_{0}^{2}\right)=\delta\left(k^{2}-Q_{0}^{2}\right)
$$

\footnotetext{
${ }^{1}$ Strictly speaking, within the leading twist collinear factorization framework this lower leg should be on-shell, so $Q_{0}^{2}$ is a regularization scale.
}

i.e., the incoming gluon has a fixed nonzero virtuality. With this definition a moment space gluon structure function can be defined as ${ }^{2}$

$$
\mathcal{G}\left(Q^{2}, N\right)=\int_{0}^{Q^{2}} \frac{d k^{2}}{k^{2}} f\left(N, k^{2}, Q_{0}^{2}\right) \times g_{B}\left(N, Q_{0}^{2}\right),
$$

where $g_{B}\left(N, Q_{0}^{2}\right)$ is the bare gluon distribution as a function of the factorization scale $Q_{0}^{2}{ }^{3}$

In order to solve this equation it is convenient to take a further Mellin transformation with respect to $k^{2}$, i.e., define

$$
\widetilde{f}(\gamma, N)=\int_{0}^{\infty} d k^{2}\left(k^{2}\right)^{-1-\gamma} f\left(k^{2}, N\right) .
$$

This leads to the BFKL equation written in the form

$$
\widetilde{f}(\gamma, N)=\widetilde{f}^{0}\left(\gamma, Q_{0}^{2}\right)+\left(\bar{\alpha}_{s} / N\right) \chi(\gamma) \widetilde{f}(\gamma, N),
$$

where $\tilde{f}^{0}\left(\gamma, Q_{0}^{2}\right)=\exp \left(-\gamma \ln \left(Q_{0}^{2}\right)\right)$ and $\chi(\gamma)$ is the characteristic function

$$
\chi(\gamma)=2 \psi(1)-\psi(\gamma)-\psi(1-\gamma) .
$$

Hence,

$$
\widetilde{f}(\gamma, N)=\frac{\tilde{f}^{0}(\gamma, N)}{1-\left(\bar{\alpha}_{s} / N\right) \chi(\gamma)} .
$$

For asymptotically small $x$ this can be accurately inverted back to $x$ and $k^{2}$ space using the saddle point technique to give the celebrated result

$$
f\left(x, k^{2}\right) \propto x^{-\lambda}\left(\frac{k^{2}}{\bar{\alpha}_{s} \ln (1 / x)}\right)^{1 / 2} \exp \left(\frac{-\ln ^{2}\left(k^{2} / Q_{0}^{2}\right)}{56 \zeta(3) \bar{\alpha}_{s} \ln (1 / x)}+\cdots\right),
$$

where $\lambda=4 \ln 2 \bar{\alpha}_{s}$ and $\cdots$ denotes subleading terms as $x$ $\rightarrow 0$. Hence, we see that the BFKL equation at LO predicts powerlike growth in $x^{-\lambda}$ and in $k^{2}$, as well as a diffusion in $k^{2}$. One can also be a little more systematic and solve for the coefficient functions and anomalous dimensions for the gluon, it is easy to generalize Eq. (2.10) to give the double Mellin space expression for the gluon structure function

$$
\widetilde{\mathcal{G}}(\gamma, N)=\frac{\tilde{f}^{0}(\gamma, N) g_{B}\left(N, Q_{0}^{2}\right)}{\gamma\left(1-\left(\bar{\alpha}_{s} / N\right) \chi(\gamma)\right)}
$$

and

\footnotetext{
${ }^{2}$ In this paper I will ignore the singlet quark distribution. This is purely for simplicity and does not change any of the conclusions at all. In most expressions the replacement of $g_{B}\left(N, Q_{0}^{2}\right)$ with $g_{B}\left(N, Q_{0}^{2}\right)+\frac{4}{9} \Sigma_{B}\left(N, Q_{0}^{2}\right)$, where $\Sigma_{B}\left(N, Q_{0}^{2}\right)$ is the bare singlet quark distribution, is all that is required to make them completely correct.

${ }^{3}$ In making this definition of the gluon distribution we have defined a factorization scheme.
} 


$$
\mathcal{G}\left(Q^{2}, N\right)=\frac{1}{2 \pi i} \int_{1 / 2-i \infty}^{1 / 2+i \infty} d \gamma \exp \left(\gamma \ln \left(Q^{2}\right)\right) \widetilde{\mathcal{G}}(\gamma, N) .
$$

From Eq. (2.12) we see that there are poles when 1 $-\left(\bar{\alpha}_{s} / N\right) \chi(\gamma)=0$. Defining the rightmost solution of this equation by

$$
\chi\left(\gamma^{0}\left(\bar{\alpha}_{s} / N\right)\right) \frac{\bar{\alpha}_{s}}{N}=1
$$

we obtain the leading twist solution for the gluon structure function

$$
\mathcal{G}\left(Q^{2}, N\right)=\frac{1}{-\left(\bar{\alpha}_{s} / N\right) \gamma^{0} \chi^{\prime}\left(\gamma^{0}\right)}\left(\frac{Q^{2}}{Q_{0}^{2}}\right)^{\gamma^{0}} g_{B}\left(N, Q_{0}^{2}\right) .
$$

Hence, $\gamma^{0}\left(\bar{\alpha}_{s} / N\right)$ is the anomalous dimension governing the $Q^{2}$ evolution of the gluon [28], and $\mathcal{R}\left(\bar{\alpha}_{s} / N\right) \equiv-\left(\bar{\alpha}_{s} /\right.$ $\left.N \gamma^{0} \chi^{\prime}\left(\gamma^{0}\right)\right)^{-1}$ is a type of coefficient function giving the normalization [25]. Each of these may be expanded as power series in $\left(\bar{\alpha}_{s} / N\right)$, which then lead to power series in $\bar{\alpha}_{s} \ln (1 / x)$ in $x$ space. Both are only convergent for $\bar{\alpha}_{s} / N$ $<4 \ln 2$, each developing a branch point showing that in $x$ space they grow like $x^{-(1)-\lambda}$. Using the saddle point technique one may find the asymptotic form of the $x$-space splitting function and coefficient function finding that

$$
P^{0}(x) \rightarrow \frac{\bar{\alpha}_{s}}{x} x^{-\lambda}\left(\frac{1}{(56 \pi \zeta(3))^{1 / 2}\left(\bar{\alpha}_{s} \ln (1 / x)\right)^{3 / 2}}\right),
$$

and

$$
R(x)=4 \ln 2 \bar{\alpha}_{s} x^{-\lambda}\left(\frac{1}{14 \pi \zeta(3) \bar{\alpha}_{s} \ln (1 / x)}\right)^{1 / 2} .
$$

Therefore, both the anomalous dimension and the coefficient function predict powerlike behavior for the gluon distribution, although the true input for the distribution is really $R(x)$ convoluted with $g_{B}\left(x, Q_{0}^{2}\right)$ of course, and this leads to the exact form of $R(x)$ being sensitive to the input $f^{0}\left(N, Q_{0}^{2}\right) .{ }^{4}$ However, this powerlike behavior does not set in until very small $x$, as may be seen by examining the terms in the expansion for each quantity in powers of $\bar{\alpha}_{s} \ln (1 / x)$.

It has long been suspected that the diffusion property of the solution to the BFKL equation may have serious consequences when working beyond the strictly LO framework $[13,22,29,20,23]$. One may appreciate this by recognizing that in the small $x$ limit, defining $\xi=\ln (1 / x)$, we may write

\footnotetext{
${ }^{4}$ In the language of the factorization theorem this translates into $R(x)$ being regularization scheme dependent, e.g., if one uses dimensional regularization rather than an off-shell gluon, $R(x)$ has a factor of $\left(\bar{\alpha}_{s} \ln (1 / x)\right)^{-3 / 4}$ rather than $\left(\bar{\alpha}_{s} \ln (1 / x)\right)^{-1 / 2}$.
}

$$
f\left(k^{2}, Q_{0}^{2}, \xi\right)=\int d r^{2} f\left(k^{2}, r^{2}, \xi^{\prime}\right) f\left(r^{2}, Q_{0}^{2}, \xi-\xi^{\prime}\right)
$$

For a given $\xi^{\prime}$ we can ask for the mean and the deviation of $\ln \left(r^{2}\right)$. This is equivalent to asking for the typical $\ln \left(k^{2}\right)$ at some point along the ladder diagram representing the function $f\left(k^{2}, Q_{0}^{2}, \xi\right)$, and also its spread, i.e., the range of important values of $q^{2}$ involved in finding the solution of the BFKL equation. The result is well known:

$$
\left\langle\ln \left(r^{2} /\left(k Q_{0}\right)\right)\right\rangle=\frac{\ln \left(k^{2} / Q_{0}^{2}\right)}{2}\left(1-2 \frac{\xi^{\prime}}{\xi}\right),
$$

and the rms deviation is

$$
\sigma^{2}=28 \zeta(3) \bar{\alpha}_{s} \xi^{\prime}\left(1-\frac{\xi^{\prime}}{\xi}\right) .
$$

So over much of the ladder $\left\langle\ln \left(r^{2}\right)\right\rangle \sim \frac{1}{2} \ln \left(k^{2} / Q_{0}^{2}\right)$ and $\sigma$ $\sim\left(14 \zeta(3) \bar{\alpha}_{s} \ln (1 / x)\right)^{1 / 2}$. Hence, for very low $x$ there will be significant diffusion into both the infrared and the ultraviolet. In the case of fixed coupling this does not cause any serious problems. However, in the case of the running coupling the size of the coupling grows quickly in the infrared, and hence this diffusion suggests that there will be serious contamination from nonperturbative physics.

Before looking at the BFKL equation for running coupling let us briefly examine the role played by the various regions of $q^{2}$ in the fixed coupling case. In order to determine the role played by the region of low transverse momentum we consider a upper cutoff of $k_{0}^{2}$ in the integral in Eq. (2.3). The only restriction we place on $k_{0}^{2}$ is that $k_{0}^{2} \ll k^{2}$ for whatever transverse momentum we ultimately wish to consider at the top of the ladder. With this restriction we see that for all momenta over which we integrate we have the relation

$$
K^{0}\left(q^{2}, k^{2}\right) f\left(q^{2}\right)=f\left(q^{2}\right)+\mathcal{O}\left(\frac{k_{0}^{2}}{k^{2}}\right),
$$

and inserting into the cutoff version of Eq. (2.3) we obtain

$\frac{\bar{\alpha}_{s}}{N} \int_{0}^{k_{0}^{2}} \frac{d q^{2}}{q^{2}} K^{0}\left(q^{2}, k^{2}\right) f\left(q^{2}\right)=\frac{\bar{\alpha}_{s}}{N} h\left(k_{0}^{2}, f\left(k^{2}<k_{0}^{2}\right)\right)+\mathcal{O}\left(\frac{k_{0}^{2}}{k^{2}}\right)$.

The integral over the region $q^{2} \leqslant k_{0}^{2} \ll k^{2}$ contributes only a constant to the right hand side of Eq. (2.3), dependent on the form of $f\left(q^{2}, N\right)$ at low momentum, but independent of the value of $k^{2}$ we consider as long as it is large. If $k^{2}$ is actually smaller than $k_{0}^{2}$ then $h\left(k_{0}^{2}, f\left(k^{2}<k_{0}^{2}\right)\right)$ becomes a much more sensitive function of $k^{2}$, and in the limit $k^{2} \rightarrow 0$ it is easy to see that it becomes equal to the value of the integral in Eq. (2.3) with no upper cutoff. Hence, $h\left(k_{0}^{2}, f\left(k^{2}<k_{0}^{2}\right)\right)$ has the same structure for $k^{2} \rightarrow 0$ as the full integral on the right hand side of Eq. (2.3), but tends to a constant function of $k_{0}^{2}$ for $k^{2} \gg k_{0}^{2}$.

Thus, if we imagine imposing an infrared cutoff on Eq. (2.3) we can simply subtract the result of the integral up to 
$k_{0}^{2}$ [now with a different $f\left(q^{2}\right)$ for low $q^{2}$, in particular the infrared cutoff renders it infrared finite] from the right hand side of Eq. (2.3), obtaining (up to higher twist corrections)

$$
\begin{aligned}
f\left(k^{2}, Q_{0}^{2}, \bar{\alpha}_{s} / N\right)= & f^{0}\left(k^{2}, Q_{0}^{2}\right)-\frac{\bar{\alpha}_{s}}{N} h\left(k^{2}, k_{0}^{2}\right) \\
& +\frac{\bar{\alpha}_{s}}{N} \int_{0}^{\infty} \frac{d q^{2}}{q^{2}} K^{0}\left(q^{2}, k^{2}\right) f\left(q^{2}\right) .
\end{aligned}
$$

Taking the Mellin transform of this equation we get

$$
\widetilde{f}(\gamma, N)=\widetilde{f}^{0}\left(\gamma, Q_{0}^{2}\right)-\left(\bar{\alpha}_{s} / N\right) \widetilde{h}\left(\gamma, k_{0}^{2}\right)+\left(\bar{\alpha}_{s} / N\right) \chi(\gamma) \widetilde{f}(\gamma, N),
$$

where $\tilde{h}\left(\gamma, k_{0}^{2}\right)$ is analytic for $\gamma>0\left[h\left(k^{2}, k_{0}^{2}\right)\right.$ tends to a constant at high $k^{2}$. This second term on the right may simply be absorbed into the definition of the input and our expression for $\widetilde{f}(\gamma, N)$ is exactly the same as in Eq. (2.10) up to this transformed input, i.e.,

$$
\widetilde{f}(\gamma, N)=\frac{\tilde{f}^{0}(\gamma, N)-\left(\bar{\alpha}_{s} / N\right) \widetilde{h}\left(\gamma, k_{0}^{2}\right)}{1-\left(\bar{\alpha}_{s} / N\right) \chi(\gamma)} .
$$

Performing the inverse Mellin transformation, then for the leading twist solution the pole is in exactly the same place and we obtain exactly the same $k^{2}$ dependence as previously, but a potentially very different $N$-dependent normalization. Hence we see that the region of transverse momentum $\ll k^{2}$ contributes very significantly to the overall normalization of our leading twist solution, but negligibly to the evolution, essentially because the contribution from the infrared region coming from the convolution in the BFKL equation is the same for all high $k^{2}$. We also notice that the other, higher twist poles found in Eq. (2.10) are now eliminated by the presence of $-\left(\bar{\alpha}_{s} / N\right) h\left(\gamma, k_{0}^{2}\right)$.

This above argument is hardly new, and much more detailed analysis can be found in [30,31] who consider the Mellin space solution carefully, showing that the infrared cutoff does indeed change only the residue of the rightmost pole in $\gamma$ (and removes all poles in the left half plane). It is also noted that infrared cutoffs influence only the normalization of the gluon distribution, leaving the shape in $x$ as well as $Q^{2}$ largely unchanged [29]. This is because the effect is to change the type of singularity in $N$-space, but not the actual position, i.e., $N=4 \ln 2 \bar{\alpha}_{s}$. However, this is not usually discussed together with the phenomenon of diffusion. In the case of fixed coupling the effect of diffusion is less important than for running coupling for the obvious reason that the coupling is the same at all scales. Nevertheless, the above arguments imply that in the case of running coupling diffusion into the infrared, i.e., strong coupling, should again only influence the normalization of the gluon, while diffusion into the ultraviolet, i.e., weak coupling, should only influence the evolution in $Q^{2}$. We will now investigate this in more detail.

\section{BFKL EQUATION FOR RUNNING COUPLING}

It was expected in [12] that the way to incorporate the running coupling into the BFKL equation was to modify Eq. (2.3) to

$$
\begin{aligned}
f\left(k^{2}, Q_{0}^{2}, \bar{\alpha}_{s}\left(k^{2}\right) / N\right)= & f^{0}\left(k^{2}, Q_{0}^{2}\right) \\
& +\frac{\bar{\alpha}_{s}\left(k^{2}\right)}{N} \int_{0}^{\infty} \frac{d q^{2}}{q^{2}} K^{0}\left(q^{2}, k^{2}\right) f\left(q^{2}\right),
\end{aligned}
$$

where

$$
\alpha_{s}=1 /\left(\beta_{0} \ln \left(k^{2} / \Lambda^{2}\right)\right),
$$

$\beta_{0}=\left(11-2 N_{f} / 3\right) /(4 \pi)$, and $N_{f}$ is the number of active flavors. One of the main results of the NLO corrections to the BFKL kernel is to show that this is indeed an effective way to account for the running coupling (this will be discussed more later). One can solve this equation in the same type of way as for the fixed coupling case, i.e., take the Mellin transformation with respect to $\left(k^{2} / \Lambda^{2}\right)$. It is most convenient to first multiply through by $\ln \left(k^{2} / \Lambda^{2}\right)$, and then obtain

$$
\frac{d \widetilde{f}(\gamma, N)}{d \gamma}=\frac{d \widetilde{f}^{0}\left(\gamma, Q_{0}^{2}\right)}{d \gamma}-\frac{1}{\bar{\beta}_{0} N} \chi(\gamma) \widetilde{f}(\gamma, N),
$$

where $\bar{\beta}_{0}=\left(\pi \beta_{0} / 3\right)$. The inclusion of the running coupling has thus completely changed the form of our double Mellin space equation, turning it from a simple equality into a first order differential equation. However, this may be easily solved to give

$$
\begin{aligned}
\widetilde{f}(\gamma, N)= & \exp \left(-X(\gamma, N) /\left(\bar{\beta}_{0} N\right)\right) \int_{\gamma}^{\infty} \frac{d \widetilde{f}_{0}\left(\widetilde{\gamma}, N, Q_{0}^{2}\right)}{d \widetilde{\gamma}} \\
& \times \exp \left(X(\widetilde{\gamma}) /\left(\bar{\beta}_{0} N\right)\right) d \widetilde{\gamma}
\end{aligned}
$$

where

$$
X(\gamma)=\int_{1 / 2}^{\gamma} \chi(\hat{\gamma}) d \hat{\gamma} \equiv\left(2 \psi(1)\left(\gamma-\frac{1}{2}\right)-\ln \left(\frac{\Gamma(\gamma)}{\Gamma(1-\gamma)}\right)\right) .
$$

The leading singularity in the $\gamma$ plane for $\exp (-X(\gamma) /$ $\left(\bar{\beta}_{0} N\right)$ ), is cancelled by an integral from $0 \rightarrow \gamma$ of the integrand depending on $\widetilde{\gamma}[15]$, and so up to higher twist corrections we may simplify (3.4) to

$$
\begin{aligned}
\widetilde{f}(\gamma, N)= & \exp \left(-X(\gamma) /\left(\bar{\beta}_{0} N\right)\right) \int_{0}^{\infty} \frac{d \widetilde{f}_{0}\left(\widetilde{\gamma}, N, Q_{0}^{2}\right)}{d \widetilde{\gamma}} \\
& \times \exp \left(X(\widetilde{\gamma}) /\left(\bar{\beta}_{0} N\right)\right) d \widetilde{\gamma}
\end{aligned}
$$

Using our previous choice of input, i.e., fixed virtuality, we obtain the moment space gluon structure function 


$$
\begin{aligned}
\mathcal{G}\left(Q^{2}, N\right)= & \frac{1}{2 \pi i} \int_{1 / 2-i \infty}^{1 / 2+i \infty} \frac{1}{\gamma} \exp \left(\gamma \ln \left(Q^{2} / \Lambda^{2}\right)\right. \\
& \left.-X(\gamma) /\left(\bar{\beta}_{0} N\right)\right) d \gamma \int_{0}^{\infty} \exp \left(-\widetilde{\gamma} \ln \left(Q_{0}^{2} / \Lambda^{2}\right)\right. \\
& \left.+X(\widetilde{\gamma}) /\left(\bar{\beta}_{0} N\right)\right) d \widetilde{\gamma} g_{B}\left(Q_{0}^{2}, N\right) \\
= & a\left(Q^{2}, N\right) b\left(Q_{0}^{2}, N\right) g_{B}\left(Q_{0}^{2}, N\right) .
\end{aligned}
$$

Hence, as in the case of fixed coupling constant, at leading twist the solution has factorized into a $Q^{2}$-dependent part $a\left(Q^{2}, N\right)$ which determines the evolution, and an input dependent part $b\left(Q_{0}^{2}, N\right)$ which can be combined with the bare input gluon distribution to provide the input for the gluon distribution $[14,15,21]$. This time the different parts are not so easy to calculate though. Clearly the behavior of both functions is determined by the form of $\exp \left(X(\gamma) /\left(\bar{\beta}_{0} N\right)\right)$, since this determines the singularity structure.

Considering $b\left(Q_{0}^{2}, N\right)$ we find that $\exp \left(X(\gamma) /\left(\bar{\beta}_{0} N\right)\right)$ has poles at all positive integers, and zeroes at 0 and all negative integers. Hence, $b\left(Q_{0}^{2}, N\right)$ is not properly defined, since the integrand has an infinite number of poles lying along the line of integration. These are due to the divergence of the coupling at low $k^{2}$ and can only be removed by some infrared regularization. Hence, the diffusion into the infrared has destroyed the apparent (limited) predictive power for the input. Imposing some regularization scale $k_{0}^{2}$ and repeating the same arguments as the previous section it is clear that up to higher twist corrections the effect of the regularization is simply to leave the factor $a\left(Q^{2}, N\right)$ unchanged, and change $b\left(Q_{0}^{2}, N\right)$ to

$$
\begin{aligned}
c\left(Q_{0}^{2}, k_{0}^{2}, N\right)= & \int_{0}^{\infty}\left(\frac{d \widetilde{f}_{0}\left(\widetilde{\gamma}, N, Q_{0}^{2}\right)}{d \widetilde{\gamma}}+\widetilde{h}\left(\widetilde{\gamma}, k_{0}^{2}\right)\right) \\
& \times \exp \left(X(\widetilde{\gamma}) /\left(\bar{\beta}_{0} N\right)\right) d \widetilde{\gamma},
\end{aligned}
$$

where the factor $\left(d \widetilde{f}_{0}\left(\tilde{\gamma} Q_{0}^{2}, N\right) / d \widetilde{\gamma}+\widetilde{h}\left(\widetilde{\gamma}, k_{0}^{2}\right)\right)$ removes the singularities in $\exp \left(X(\widetilde{\gamma}) /\left(\bar{\beta}_{0} N\right)\right)$. Thus, we have

$$
\mathcal{G}\left(Q^{2}, N\right)=a\left(Q^{2}, N\right) c\left(Q_{0}^{2}, k_{0}^{2}, N\right) g_{B}\left(Q_{0}^{2}, k_{0}^{2}, N\right),
$$

as a well-defined solution. ${ }^{5}$ For a given regularization one can solve for $c\left(Q_{0}^{2}, k_{0}^{2}, N\right)$, as has been done numerically, ${ }^{6}$ generally obtaining some powerlike growth in $x$ space, but which is totally dependent on the type and scale of regularization $[16,17,20,32]$. No real predictive power remains (this will be discussed more in Sec. V).

\footnotetext{
${ }^{5}$ That the solution at leading twist is of this general form was shown in [21] by putting the BFKL equation with running coupling in the form of an infinite order differential equation with effective potential depending on the low $k^{2}$ regularization of the coupling.

${ }^{6}$ The numerical solutions are always for the whole of the gluon structure function, not just $c\left(Q_{0}^{2}, k_{0}^{2}, N\right)$.
}

Even without regularization there is no obstruction to solving for the $Q^{2}$ dependent part of the gluon distribution, and this is unchanged by this regularization, i.e., is unaffected, up to $\mathcal{O}\left(k_{0}^{2} / Q^{2}\right)$ corrections, by the diffusion into the infrared. The function $a\left(Q^{2}, N\right)$ is, of course, determined by the singularities of $\exp \left(-X(\gamma) /\left(\bar{\beta}_{0} N\right)\right)$ in the $\gamma$ plane. Here we notice a fundamental difference between the cases of the fixed and running couplings. Whereas previously the leading singularity was a pole at $\left(\widetilde{\alpha}_{s} / N\right) \chi(\gamma)=1$, i.e., at $\gamma \rightarrow \frac{1}{2}$ as $N \rightarrow 4 \ln 2 \bar{\alpha}_{s}$, now the leading singularity is an essential singularity at $\gamma=0$ : there is no powerlike behavior in $Q^{2}$. Similarly, the branch point in the $N$ plane at $4 \ln 2 \bar{\alpha}_{s}$ has become an essential singularity at $N=0$ : there is no powerlike behavior in $x$. The introduction of the running of the coupling has therefore also had an extreme effect upon the evolution, changing its character completely. This point has been noticed before $[15,20]$, but not emphasized or studied in detail. Hence I stress the fundamental results of introducing a running coupling: the $Q^{2}$-independent part of the solution is formally divergent, and hence is totally regularization scheme dependent: the $Q^{2}$-dependent part has no powerlike growth in $x$.

In fact we can obtain some information about the $x$ behavior by noting that we can find the inverse Laplace transformation of $\exp \left(-X(\gamma) /\left(\bar{\beta}_{0} N\right)\right)$ precisely $[20,8]$. It is a standard result that

$$
\frac{1}{2 \pi i} \int_{-i \infty}^{i \infty} \exp (N \xi+K / N) d N=(A / \xi)^{1 / 2} I_{1}(2(A \xi))^{1 / 2}
$$

where $I_{1}(z)$ is the modified Bessel function, which for large values of its argument $\rightarrow \exp (z) /(2 \pi z)^{1 / 2}$. Hence for large $\xi$

$$
a(\xi, \gamma) \sim\left(-X(\gamma) / \bar{\beta}_{0} \xi\right)^{3 / 4} \exp \left(2\left[-\xi X(\gamma) / \bar{\beta}_{0}\right]\right)^{1 / 2} .
$$

It is difficult to perform the inverse Mellin transformation to get the $Q^{2}$ dependence, but the leading singularity is at $\gamma$ $=0$. Thus, for any $Q^{2}$ the leading twist solution for $a\left(\xi, Q^{2}\right)$ must have small $x$ behavior going like $\exp \left(\xi^{1 / 2}\right)$ rather than the $\exp (\lambda \xi)$ for the fixed coupling case. This is easy to understand in terms of the diffusion picture. Since the function $a\left(\xi, Q^{2}\right)$ is insensitive to the diffusion towards the infrared, but sensitive to that into the ultraviolet, we expect the typical scale in the process to be determined by this latter diffusion. Thus the typical scale for the process will be approximately set by $\ln \left(\widetilde{k}^{2}\right) \sim \ln \left(k^{2}\right)+\sigma \sim \ln \left(k^{2}\right)+4\left(\bar{\alpha}_{s}\left(k^{2}\right) \xi\right)^{1 / 2}$. Hence, the effective strength of the running coupling will be set by $\widetilde{k}^{2}$, rather than $k^{2}$, and as $x \rightarrow 0$ we will have $\bar{\alpha}_{s}^{\text {eff }} \sim 1 /(\xi)^{1 / 2}$. This type of effective coupling has precisely the effect of turning the low $x$ behavior of the fixed coupling solution to that which we find for the running coupling. Hence, the diffusion into the ultraviolet has a major impact on the $Q^{2}$ dependent part of our gluon distribution, but in a well controlled, and in principle calculable way, unlike the effect of the infrared diffusion on the $Q_{0}^{2}$ dependent input. 


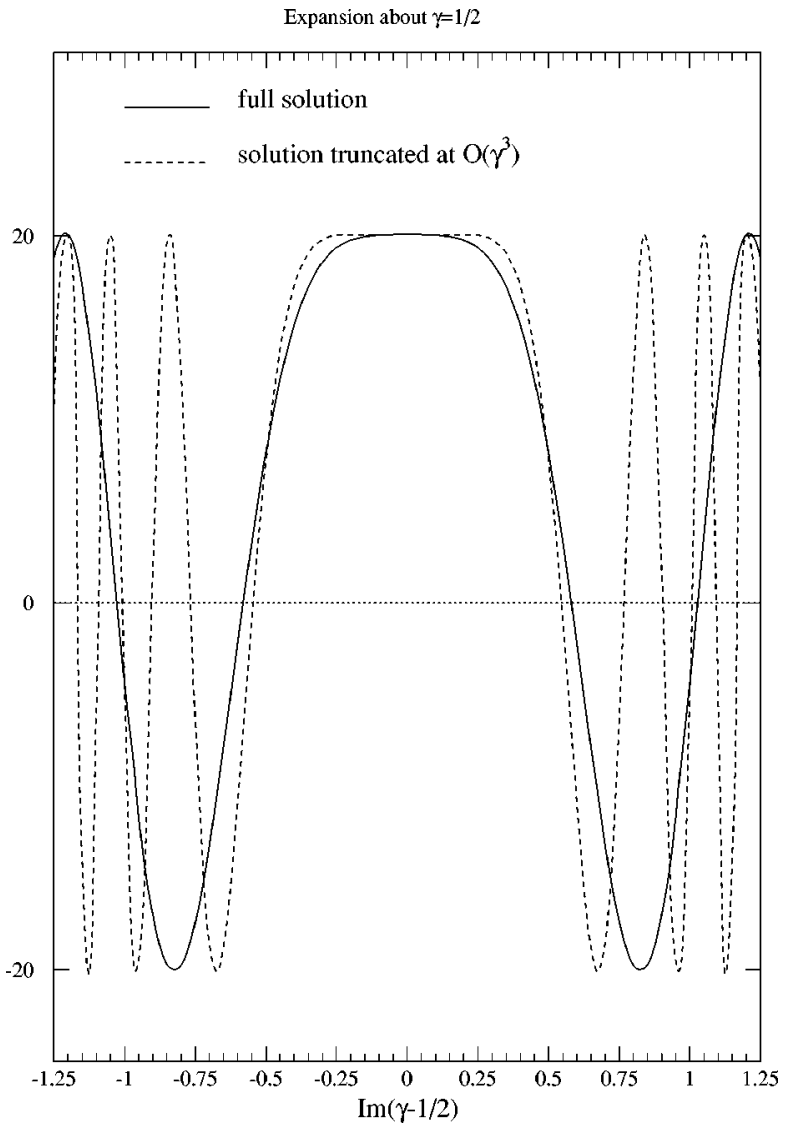

FIG. 1. Comparison of the full function $\exp \left(\gamma \ln \left(Q^{2} / \Lambda^{2}\right)\right.$ $\left.-X(\gamma) /\left(\bar{\beta}_{0} N\right)\right)$ to the case where the exponent is truncated at $\mathcal{O}\left(\gamma^{3}\right)$ along the line $\mathfrak{R}(\gamma)=\frac{1}{2}$. I choose $\ln \left(Q^{2} / \Lambda^{2}\right)=6$ and $1 /\left(\bar{\beta}_{0} N\right)=2$.

Of course, this is just a qualitative argument giving only the general form of the results. It is also for the function $a\left(x, Q^{2}\right)$, which must be convoluted with an unknown, if $Q^{2}$-independent input function in order to obtain physical results. It would be nice to be more quantitative, and also to calculate some physical quantity unambiguously. For example, staying in moment space we can examine $\left[d \mathcal{G}\left(N, Q^{2}\right) / d \ln \left(Q^{2}\right)\right]$, which is an entirely perturbatively calculable quantity, and its transformation into $x$ space. This will be considered in the next section.

\section{SOLVING THE BFKL SOLUTION FOR RUNNING COUPLING： EVOLUTION}

The usual approaches taken to finding the solution for $a\left(Q^{2}, N\right)$ (or the full solution) are to assume that for small $x$ one can expand $X(\gamma)$ about $\gamma=\frac{1}{2}$ to some finite order in $\gamma^{7}$ usually to $\mathcal{O}\left(\gamma^{3}\right)$, or to use the saddle point method. Neither of these are at all accurate unless $Q^{2}$ is very large indeed. This is because along a line parallel to the imaginary axis

\footnotetext{
${ }^{7}$ This is equivalent to writing the $k^{2}$-space BFKL equation as an infinite order differential equation and truncating at a low order in derivatives, or iterating the LO solution in the truncated form (2.12) in the NLO equation.
}

$X(\gamma)$ is not at all well represented by the first few terms in a power series in $\gamma$ about either $\gamma=\frac{1}{2}$ or about the saddle point. The former can be seen in Fig. 1, where we compare the the full function $\exp \left(\gamma \ln \left(Q^{2} / \Lambda^{2}\right)-X(\gamma) /\left(\bar{\beta}_{0} N\right)\right)$ to the case where the exponent is truncated at $\mathcal{O}\left(\gamma^{3}\right)$ along the line $\mathfrak{R}(\gamma)=\frac{1}{2} .{ }^{8}$ Clearly the integral over the two contours need bear little similarity.

When using the saddle point technique one finds the minimum of the exponent of the integrand in the definition of $a\left(Q^{2}, N\right)$ and expands in a Taylor series about this point. This minimum occurs when

$$
\frac{d}{d \gamma}\left(\gamma \ln \left(Q^{2} / \Lambda^{2}\right)-X(\gamma) /\left(\bar{\beta}_{0} N\right)\right)=0,
$$

which using the definition (3.5) leads to

$$
\frac{1}{\bar{\beta}_{0} \ln \left(Q^{2} / \Lambda^{2}\right) N} \chi(\bar{\gamma}) \equiv \frac{\bar{\alpha}_{s}\left(Q^{2}\right)}{N} \chi(\bar{\gamma})=1,
$$

i.e., at $\bar{\gamma}=\gamma^{0}\left(\bar{\alpha}_{s}\left(Q^{2}\right) / N\right)$, the anomalous dimension for the fixed coupling case, but with the running coupling evaluated at scale $Q^{2}$. The integrand defining $a\left(Q^{2}, N\right)$ is thus evaluated along the axis $\mathfrak{R}(\gamma)=\gamma^{0}\left(\bar{\alpha}_{s}\left(Q^{2}\right) / N\right)$, i.e.,

$$
\begin{aligned}
a\left(Q^{2}, N\right)= & \frac{1}{2 \pi i} \exp \left(\int Q^{2} \gamma^{0}\left(\bar{\alpha}_{s}\left(q^{2}\right) / N\right) d \ln q^{2}\right) \\
& \times \int_{-i \infty}^{i \infty} \frac{1}{\gamma^{0}+\gamma} \exp \left(\gamma \ln \left(Q^{2} / \Lambda^{2}\right)\right. \\
& \left.+\left[X\left(\gamma_{0}\right)-X\left(\gamma^{0}+\gamma\right)\right] /\left(\bar{\beta}_{0} N\right)\right) d \gamma .
\end{aligned}
$$

Letting, $\gamma \rightarrow-i \gamma$ and expanding about $\gamma^{0}\left(\bar{\alpha}_{s} / N\right)$ this becomes

$$
\begin{aligned}
a\left(Q^{2}, N\right)= & \frac{1}{2 \pi} \exp \left(\int Q^{2} \gamma^{0}\left(\bar{\alpha}_{s}\left(q^{2}\right) / N\right)\right) d \ln q^{2} \\
& \times \int_{-\infty}^{\infty}\left(\frac{1}{\gamma^{0}}+\cdots\right) \exp \left(\gamma^{2} \chi^{\prime}\left(\gamma^{0}\right) /\left(2 \bar{\beta}_{0} N\right)\right. \\
& +\cdots) d \gamma .
\end{aligned}
$$

This is then normally evaluated by ignoring all those parts not explicitly included above, and performing the Gaussian integral [33] obtaining $^{9}$

$$
\begin{aligned}
a^{S P}\left(Q^{2}, N\right)= & \frac{1}{\gamma^{0}\left(\bar{\alpha}_{s}\left(Q^{2}\right) / N\right)\left[-\chi^{\prime}\left(\gamma^{0}\left[\bar{\alpha}_{s}\left(Q^{2}\right) / N\right]\right)\right]^{1 / 2}} \\
& \times \exp \left(\int Q^{Q^{2}} \gamma^{0}\left(\bar{\alpha}_{s}\left(q^{2}\right) / N\right) d \ln q^{2}\right)
\end{aligned}
$$

\footnotetext{
${ }^{8}$ Actually I plot the real part of the functions. The imaginary part is odd and integrates to zero.

${ }^{9} \mathrm{~A}$ factor of $\left(\bar{\beta}_{0} N / 2 \pi\right){ }^{1 / 2}$ is absorbed into $b\left(Q_{0}^{2}, N\right)$.
} 


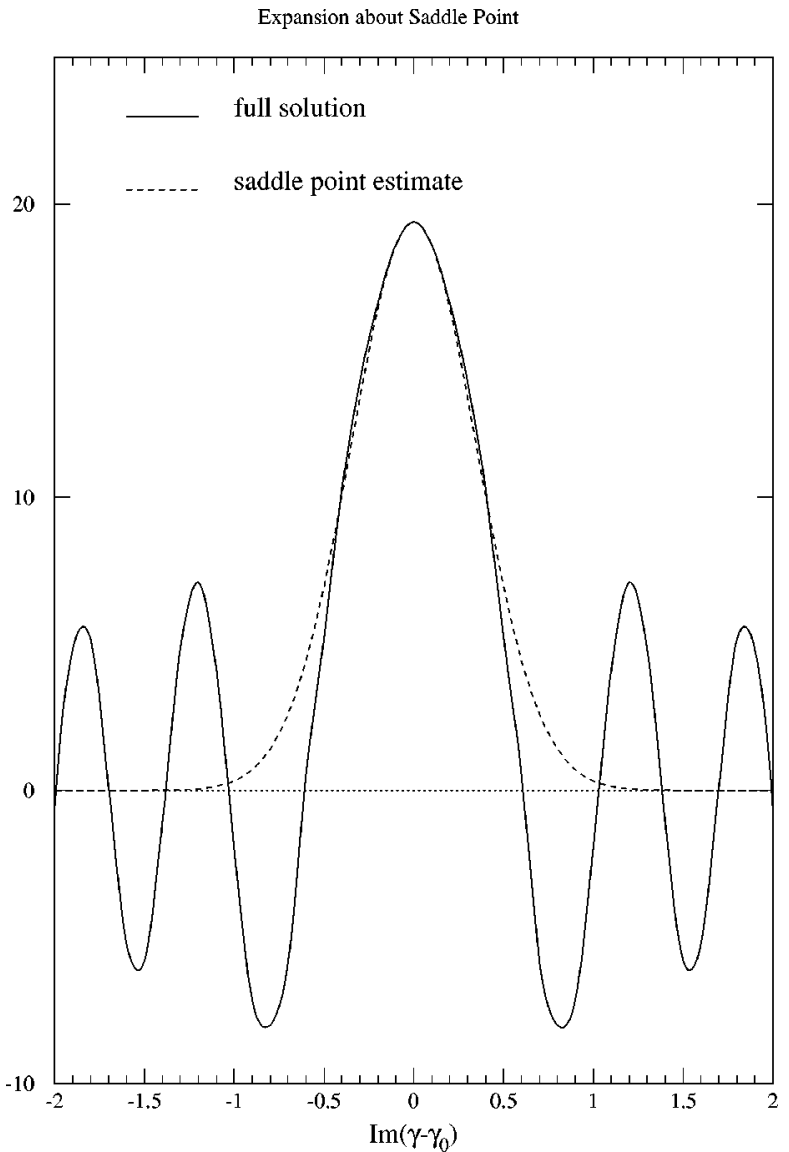

FIG. 2. Comparison of the full function $\exp \left(\gamma \ln \left(Q^{2} / \Lambda^{2}\right)\right.$ $\left.-X(\gamma) /\left(\bar{\beta}_{0} N\right)\right)$ to the function appearing in the saddle point estimate along the line $\mathfrak{R}(\gamma)=\gamma^{0}\left(\bar{\alpha}_{s} / N\right)$. I choose $\ln \left(Q^{2} / \Lambda^{2}\right)=6$ and $1 /\left(\bar{\beta}_{0} N\right)=2$, so $\gamma^{0}\left(\bar{\alpha}_{s} / N\right)=0.384$.

This is of the same form as Eq. (2.15), i.e., an evolution term governed by the previous anomalous dimension and a coefficient function which is a power series in $\bar{\alpha}_{s} / N$, except that now $\alpha_{s}$ runs with $Q^{2}$ rather than being fixed. This could be taken to imply that one can simply extract anomalous dimensions and coefficient functions from this solution and that the appropriate scale to use for the coupling is $Q^{2}$.

The invalidity of this assumption is related to the fact that Eq. (4.5) is in fact a very poor approximation to the full solution for $a\left(Q^{2}, N\right)$. This is clear because in $x$-space both the perceived anomalous dimension and coefficient function above grow like $x^{(-1)-4 \ln 2 \bar{\alpha}_{s}\left(Q^{2}\right)}$ as $x \rightarrow 0$, whereas we know that the complete solution for $a\left(Q^{2}, x\right)$ has no real powerlike behavior in $x$. We can see how we have obtained such a poor approximation by using the saddle point technique if we examine the form of the complete integrand along our contour of integration compared with the function we have actually integrated making the approximation in Eq. (4.4). This is seen in Fig. 2, ${ }^{10}$ and it is glaringly obvious that the saddle point estimate is not at all reliable in this case. Formally the corrections ignored in evaluating Eq. (4.4) are of higher or-

\footnotetext{
${ }^{10}$ Again I plot only the real part.
}

der in $\beta_{0} \alpha_{s}\left(Q^{2}\right)$ than the terms calculated, but their coefficients grow quickly, i.e., like factorials, and to be precise they are powers of $\beta_{0} \alpha_{s}\left(Q^{2}\right)\left(\bar{\alpha}_{s}\left(Q^{2}\right) \xi\right)^{r}$ higher than the presented results, where $r$ is a positive number, and are thus dominant for low enough $x$. Hence a resummation is really necessary for a true understanding.

However, an alternative view of the result in Eq. (4.5) may lead us towards the correct physics. It is not really useful to interpret the prefactor in this equation as a coefficient function which tells us something about the normalization of the gluon structure function since $a\left(N, Q^{2}\right)$ must be multiplied by an unknown $N$-dependent function in order to obtain this distribution. Rather, it is better to acknowledge that the only real information contained in $a\left(N, Q^{2}\right)$ is on the evolution of the structure function, i.e.,

$$
\frac{d \ln \mathcal{G}\left(N, Q^{2}\right)}{d \ln \left(Q^{2}\right)}=\frac{d \ln a\left(N, Q^{2}\right)}{d \ln \left(Q^{2}\right)} \equiv \Gamma\left(N, Q^{2}\right) .
$$

Thus, using $a\left(N, Q^{2}\right)$ in Eq. (4.6) gives us an entirely perturbative effective anomalous dimension governing the evolution of the gluon distribution. Using Eq. (4.5) we obtain

$$
\begin{aligned}
\Gamma\left(N, Q^{2}\right)= & \gamma^{0}\left(\bar{\alpha}_{s}\left(Q^{2}\right) / N\right)-\beta_{0} \alpha_{s}\left(Q^{2}\right) \\
& \times\left(\frac{d \gamma^{0}}{d \ln \left(\alpha_{s}\right)}\left(\frac{-\chi^{\prime \prime}\left(\gamma^{0}\right)}{2 \chi^{\prime}\left(\gamma^{0}\right)}-\frac{1}{\gamma^{0}}\right)\right) \\
& +\mathcal{O}\left(\left[\beta_{0} \alpha_{s}\left(Q^{2}\right)\right]^{2}\right) r\left(\bar{\alpha}_{s}\left(Q^{2}\right) / N\right) .
\end{aligned}
$$

So within the framework of the LO BFKL equation with running coupling our unambiguous effective anomalous dimension is the naive leading order result with coupling at scale $Q^{2}$ plus a series of corrections going like powers of $\beta_{0} \alpha_{s}\left(Q^{2}\right)$.

It is tempting to interpret the whole solution for $\Gamma\left(\alpha_{s}\left(Q^{2}\right), N\right)$ as simply telling us the appropriate scale to use in the coupling constant for the normal LO result. Indeed, this is the philosophy in the BLM scheme [24] for scale fixing which uses the NLO $\beta_{0}$-dependent corrections for any process to determine the scale to use for the coupling in the LO expression. However, in this case of an anomalous dimension for a structure function we have to decide whether it is appropriate to do this in $N$-space or $x$ space, i.e., should we write

$$
\frac{d \mathcal{G}\left(N, Q^{2}\right)}{d \ln Q^{2}} \approx \Gamma\left(N, \bar{\alpha}_{s}\left[s(N) Q^{2}\right]\right) G\left(N, Q^{2}\right)
$$

or

$$
\frac{d G\left(x, Q^{2}\right)}{d \ln \left(Q^{2}\right)} \approx \int_{x}^{1} P\left(z, \bar{\alpha}_{s}\left[s(z), Q^{2}\right]\right) G\left(x / z, Q^{2}\right) d z .
$$

Since the moment space expressions are less physical, being defined only by analytic continuations over much of the 
$N$-plane we choose the latter. ${ }^{11}$ As we will see later, this decision is backed up by higher order calculations. Note that both $\Gamma\left(N, Q^{2}\right)$ and $P\left(x, Q^{2}\right)$ are entirely independent of factorization scale, and are functions only of renormalization scale. Indeed, if there were a direct probe of the gluon, i.e., $G\left(x, Q^{2}\right)$ were directly measurable, then both $\Gamma\left(N, Q^{2}\right)$ and $P\left(x, Q^{2}\right)$ would be physically defined quantities. As such the choice of the renormalization scale is entirely open.

The simplest thing we can do is to choose the scale for the coupling constant in the leading order expression so that the NLO term in the $x$-space version of Eq. (4.7) is exactly produced by the expansion about $\alpha_{s}\left(Q^{2}\right)$. Thus, writing this $x$-space expression as

$$
\begin{aligned}
\left(x / \bar{\alpha}_{s}\left(Q^{2}\right)\right) P\left(x, Q^{2}\right)= & p^{0}\left(\bar{\alpha}_{s}\left(Q^{2}\right) \xi\right) \\
& -\beta_{0} \alpha_{s}\left(Q^{2}\right) \hat{p}^{1}\left(\bar{\alpha}_{s}\left(Q^{2}\right) \xi\right) \\
& +\mathcal{O}\left(\left[\beta_{0} \alpha_{s}\left(Q^{2}\right)\right]^{2}\right) r\left(\bar{\alpha}_{s}\left(Q^{2}\right) \xi\right),
\end{aligned}
$$

is the same as

$$
\begin{aligned}
& {\left[x / \bar{\alpha}_{s}\left(Q^{2} s\left[\xi \bar{\alpha}_{s}\left(Q^{2}\right)\right]\right)\right] P\left(x, Q^{2}\right)=p^{0}\left[\bar{\alpha}_{s}\left(Q^{2} s\left[\xi \bar{\alpha}_{s}\left(Q^{2}\right)\right]\right)\right]} \\
& \quad+\mathcal{O}\left(\left[\beta_{0} \alpha_{s}\left(Q^{2}\right)\right]^{2}\right) \hat{r}\left(\bar{\alpha}_{s}\left(Q^{2}\right) \xi\right),
\end{aligned}
$$

if we choose

$$
\exp \left(s\left[\xi \bar{\alpha}_{s}\left(Q^{2}\right)\right]\right)=\frac{\hat{p}^{1}\left(\bar{\alpha}_{s}\left(Q^{2}\right) \xi\right)}{\left(d p^{0}\left[\bar{\alpha}_{s}\left(Q^{2}\right) \xi\right] / d \ln \alpha_{s}\left(Q^{2}\right)\right)}
$$

This is the usual Brodsky-Lepage-Mackenzie (BLM) scale fixing, but here we have extra information since, in principle at least, we know higher order terms and we would expect $\hat{r}\left(\bar{\alpha}_{s}\left(Q^{2}\right) \xi\right)$ to be small if the scale fixing is correct.

Equation (4.12) can be solved for arbitrary $x$, but it is first useful to examine the limit of $x \rightarrow 0$ in order to see if our previous expectations based on qualitative arguments are confirmed. Hence we need each of the terms in Eq. (4.12) in this limit. As $x \rightarrow 0$,

$$
p^{0}\left(\bar{\alpha}_{s}\left(Q^{2}\right) \xi\right) \rightarrow \frac{1}{(56 \pi \zeta(3))^{1 / 2}} \exp \left(\lambda\left(Q^{2}\right) \xi\right)\left(\bar{\alpha}_{s}\left(Q^{2}\right) \xi\right)^{-3 / 2}
$$

and therefore

$$
\frac{d p^{0}\left(\bar{\alpha}_{s}\left(Q^{2}\right) \xi\right)}{d \ln \left(\alpha_{s}\left(Q^{2}\right)\right)} \rightarrow \frac{4 \ln 2}{(56 \pi \zeta(3))^{1 / 2}} \exp (\lambda \xi)\left(\bar{\alpha}_{s}\left(Q^{2}\right) \xi\right)^{-1 / 2}
$$

\footnotetext{
${ }^{11}$ Fixing the scale in $N$-space would lead to a scale which was singular at $N=\lambda\left(Q^{2}\right)$, which does not seem a sensible proposition, while in $x$ space it is a smooth function of $x$ as we will see.
}

In order to calculate the $x \rightarrow 0$ limit of $\hat{p}^{1}\left(\bar{\alpha}_{s}\left(Q^{2}\right) \xi\right)$ it is easiest to first consider its moment space analogue, i.e., the second term on the right of Eq. (4.7). First we note that using Eq. (2.14),

$$
\frac{d \gamma^{0}\left(\bar{\alpha}_{s}\left(Q^{2}\right) / N\right)}{d \ln \left(\alpha_{s}\left(Q^{2}\right)\right)}=-\frac{\chi\left(\gamma^{0}\left[\bar{\alpha}_{s}\left(Q^{2}\right) / N\right]\right)}{\chi^{\prime}\left(\gamma^{0}\left[\bar{\alpha}_{s}\left(Q^{2}\right) / N\right]\right)} .
$$

In the limit $x \rightarrow 0, \chi\left(\gamma^{0}\right) \rightarrow 4 \ln 2$ and $\gamma^{0} \rightarrow \frac{1}{2}$, but to be more precise,

$$
\chi\left(\gamma^{0}\right) \rightarrow 4 \ln 2-14 \zeta(3)\left(\frac{1}{2}-\gamma^{0}\right)^{2}+\cdots .
$$

Therefore,

$$
\chi^{\prime}\left(\gamma^{0}\right) \rightarrow-28 \zeta(3)\left(\frac{1}{2}-\gamma^{0}\right) \equiv-28 \zeta(3) \delta \gamma^{0} .
$$

Hence,

$$
\frac{d \gamma^{0}}{d \ln \left(\alpha_{s}\right)}=-\frac{\chi\left(\gamma^{0}\right)}{\chi^{\prime}\left(\gamma^{0}\right)} \rightarrow \frac{\ln 2}{7 \zeta(3) \delta \gamma^{0}} .
$$

Since $\delta \gamma^{0}$ is vanishingly small as $x \rightarrow 0$ we see that the $1 / \gamma^{0}$ term in Eq. (4.7) becomes subleading to the $\chi\left(\gamma^{0}\right) / \chi^{\prime}\left(\gamma^{0}\right)$ term. The $N$-space version of $\hat{p}^{1}\left(\bar{\alpha}_{s}\left(Q^{2}\right) \xi\right)$ is thus $\chi\left(\gamma^{0}\right) \chi^{\prime \prime}\left(\gamma^{0}\right) / 2\left(\chi^{\prime}\left(\gamma^{0}\right)^{2}\right)$. To progress further we need $\delta \gamma^{0}$ as a function of $N$. This can be obtained by solving Eq. (2.14) using Eq. (4.16). This gives

$$
\delta \gamma^{0}\left(\bar{\alpha}_{s}\left(Q^{2}\right) / N\right)=\left(\frac{2 \ln 2}{7 \zeta(3)}\right)^{1 / 2}\left(\frac{N}{\lambda\left(Q^{2}\right)}-1\right)^{1 / 2} .
$$

This can be substituted into the moment space analogue of $\hat{p}^{1}\left(\bar{\alpha}_{s}\left(Q^{2}\right) \xi\right)$ and the inverse transformation performed to give

$$
\hat{p}^{1}\left(\bar{\alpha}_{s}\left(Q^{2}\right) \xi\right) \rightarrow \ln 2 \exp \left(\lambda\left(Q^{2}\right) \xi\right) .
$$

This now makes it trivial to solve Eq. (4.12), and we find that in the coupling in our LO splitting function

$$
\begin{aligned}
\ln \left(Q^{2} / \Lambda^{2}\right) \rightarrow & \ln \left(\widetilde{Q}^{2} / \Lambda^{2}\right)=\ln \left(Q^{2} / \Lambda^{2}\right) \\
& +\frac{(56 \zeta(3) \pi)^{1 / 2}}{4}\left(\bar{\alpha}_{s}\left(Q^{2}\right) \xi\right)^{1 / 2} .
\end{aligned}
$$

This is exactly the sort of scale change we would expect from the diffusion into the ultraviolet. It also leads to $x P\left(\bar{\alpha}_{s}\left(Q^{2}\right), x\right) \sim \exp \left(1.14\left[\xi / \bar{\alpha}_{s}\left(Q^{2}\right)\right]^{1 / 2}\right)$ as $x \rightarrow 0$, precisely the sort of behavior we would expect from the qualitative discussions in the last section.

We can also solve Eq. (4.12) exactly rather than relying on asymptotic limits using the power series expansions of $d p^{0}\left(\bar{\alpha}_{s}\left(Q^{2}\right) \xi\right) / d \ln \left(\alpha_{s}\left(Q^{2}\right)\right)$ and $\hat{p}^{1}\left(\bar{\alpha}_{s}\left(Q^{2}\right) \xi\right)$ in $\bar{\alpha}_{s}\left(Q^{2}\right) \xi$. The results of such solutions are shown in Fig. 3, where I plot the effective coupling constant for $N_{f}=4$ derived as a function of $x$ compared to its constant value taking $Q^{2}$ as the scale. The qualitative result is entirely consistent with Eq. (4.21) though the effective scale is a little smaller than this 

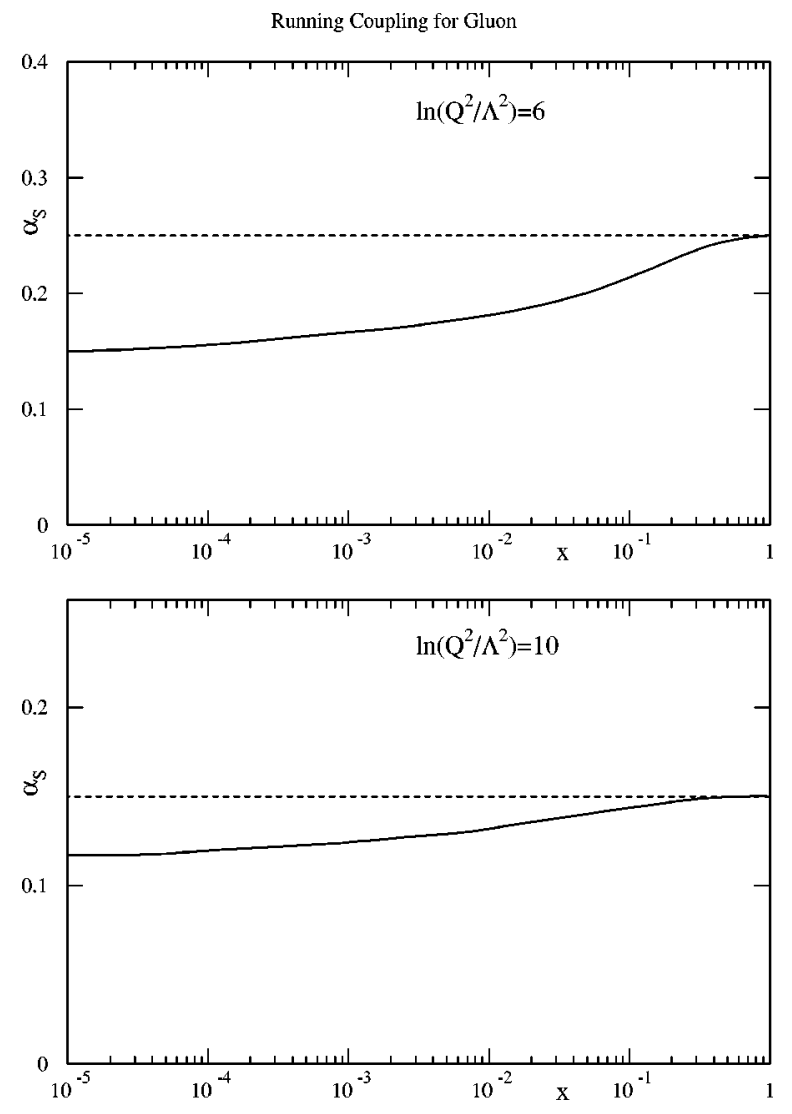

FIG. 3. The effective coupling constant for $N_{F}=4$ for the gluon structure function as a function of $x$ compared to the constant values at the relevant values of $\ln \left(Q^{2} / \Lambda^{2}\right)$.

asymptotic result at slightly high $x$ values due to $p^{0}\left(\alpha_{s}\left(Q^{2}\right) \xi\right)$ and $\hat{p}^{1}\left(\alpha_{s}\left(Q^{2}\right) \xi\right)$ not yet having reached their asymptotic expressions.

Hence, this BLM scale fixing procedure leads to a choice of scale which fits in well with our naive expectations, and must be at least broadly qualitatively correct since it does destroy the powerlike behavior we get from fixed order calculations in $\alpha_{s}\left(Q^{2}\right)$. Ignoring for the moment the fact that we have assumed the manner in which to take account of running coupling effects in the BFKL equation (we will discuss possible corrections later), we would still like to know whether our prescription is a true representation of the full effect of the running coupling, i.e., whether $\hat{r}\left(\bar{\alpha}_{s}\left(Q^{2}\right) \xi\right)$ in Eq. (4.11) is really small. At each order in $\beta_{0} \alpha_{s}\left(Q^{2}\right)$ it is possible to calculate the leading behavior in the limit $x \rightarrow 0$. By power counting one can see that these leading terms come from keeping only the next term not explicitly shown in the exponential in Eq. (4.4), i.e., the leading behavior is given by

$$
\begin{aligned}
a\left(Q^{2}, N\right)= & \frac{1}{2 \pi \gamma^{0}} \exp \left(\int Q^{2} \gamma^{0}\left(\bar{\alpha}_{s}\left(q^{2}\right) / N\right)\right) d \ln q^{2} \\
& \times \int_{-\infty}^{\infty} \exp \left(( \overline { \beta } _ { 0 } N ) ^ { - 1 } \left[\frac{1}{2} \gamma^{2} \chi^{\prime}\left(\gamma^{0}\right)\right.\right. \\
& \left.\left.+(i / 3) \gamma^{3} \chi^{\prime \prime}\left(\gamma^{0}\right)\right]\right) d \gamma .
\end{aligned}
$$

Expanding the exponential in $i \gamma^{3}$ and performing each relevant integral gives the most singular behavior in $(N$ $\left.-\lambda\left(Q^{2}\right)\right)$ at each order in $\beta_{0} \alpha_{s}\left(Q^{2}\right)$. This is a series of the form

$$
\begin{aligned}
a^{S P}\left(Q^{2}, N\right) \sum_{n=0}^{\infty}(-1)^{n} L_{n}\left(\beta_{0} \alpha_{s}\left(Q^{2}\right)\right)^{n} \\
\times\left(\lambda\left(Q^{2}\right) /\left[N-\lambda\left(Q^{2}\right)\right]\right)^{3 n / 2},
\end{aligned}
$$

where asymptotically $L_{n} \sim(1.84)^{n} n$ !. Inserting this into Eq. (4.6) and performing the transformation into $x$ space leads to a power series of the form

$$
\begin{aligned}
p^{0}\left(x, \bar{\alpha}_{s}\left(Q^{2}\right)\right) \sum_{n=0}^{\infty}(-1)^{n} A_{n}\left(\beta_{0} \alpha_{s}\left(Q^{2}\right)\right)^{n} \\
\times\left(\lambda\left(Q^{2}\right) \alpha_{s}^{1 / 2}\left(Q^{2}\right) \xi^{3 / 2}\right)^{n},
\end{aligned}
$$

where the $A_{n}$ are all positive. If $A_{n}$ were equal to $3.63^{n} / n$ ! the above series would simply be $\exp \left(-\lambda\left(Q^{2}\right) 3.63 \beta_{0} \alpha_{s}^{3 / 2}\left(Q^{2}\right) \xi^{3 / 2}\right)$, which would be precisely the leading correction in the exponent of $p^{0}\left(x, \bar{\alpha}_{s}\left(Q^{2}\right)\right)$ expected using my choice of scale, i.e.,

$$
\begin{aligned}
\exp \left(\lambda\left(Q^{2}\right) \xi\right) \rightarrow & \exp \left(\lambda\left(Q^{2}\right) \xi-\lambda\left(Q^{2}\right)\right. \\
& \left.\times \xi\left[3.63 \beta_{0} \alpha_{s}^{3 / 2}\left(Q^{2}\right) \xi^{1 / 2}\right]+\cdots\right) .
\end{aligned}
$$

In practice this works reasonably well. $A_{1}=3.63$ of course, since this set our scale. $A_{2}=7.08$ rather than 6.59 , and the terms then slowly increase above $(3.63)^{n} / n !$. As $n \rightarrow \infty$, $A_{n+1} / A_{n} \rightarrow 1.67 / n^{1 / 2}$, and therefore Eq. (4.24) cannot be precisely of the suggested form. Nevertheless, it defines a convergent series in $\left(\beta_{0} \alpha_{s}\left(Q^{2}\right)\right)^{n}\left(\lambda\left(Q^{2}\right) \bar{\alpha}_{s}^{1 / 2}\left(Q^{2}\right) \xi^{3 / 2}\right)$ which for a wide range of values mimics the desired exponential $\exp \left(-\lambda\left(Q^{2}\right) \xi\left[3.63 \beta_{0} \alpha_{s}^{3 / 2}\left(Q^{2}\right) \xi^{1 / 2}\right]\right)$ well.

This above check is not really terribly useful since the right hand side of Eq. (4.25) hardly matches $\exp \left(\lambda\left(\widetilde{Q}^{2}\right) \xi\right)$ well for very large $\xi$, and many other terms are important at all $\xi$. Including our scale choice in the LO expression for the splitting function also leads to terms not explicitly shown in Eq. (4.25) [and in the expansion of the unexponentiated terms in $\left.p^{0}\left(x, \bar{\alpha}_{s}\left(Q^{2}\right)\right)\right]$ which are subleading in $\xi$ at each power of $\beta_{0} \alpha_{s}\left(Q^{2}\right)$ to those discussed above. There are also terms of this type generated by the subasymptotic corrections to Eq. (4.21). In principle one could compare with terms generated form a more careful solution of Eq. (4.4), including also the nonleading parts coming from Eq. (4.22). This rapidly becomes extremely complicated indeed. It appears as though the logarithm of the splitting function is indeed an oscillating power series in $\beta_{0} \alpha_{s}\left(Q^{2}\right)\left(\alpha_{s}\left(Q^{2}\right) \xi\right)^{1 / 2}$, but it is difficult to prove this rigorously. [We do know that the series will converge, or at least be unambiguously summable, since the integral defining $a\left(Q^{2}, N\right)$ is well defined.] The best check to be done at the moment is to calculate the whole of 


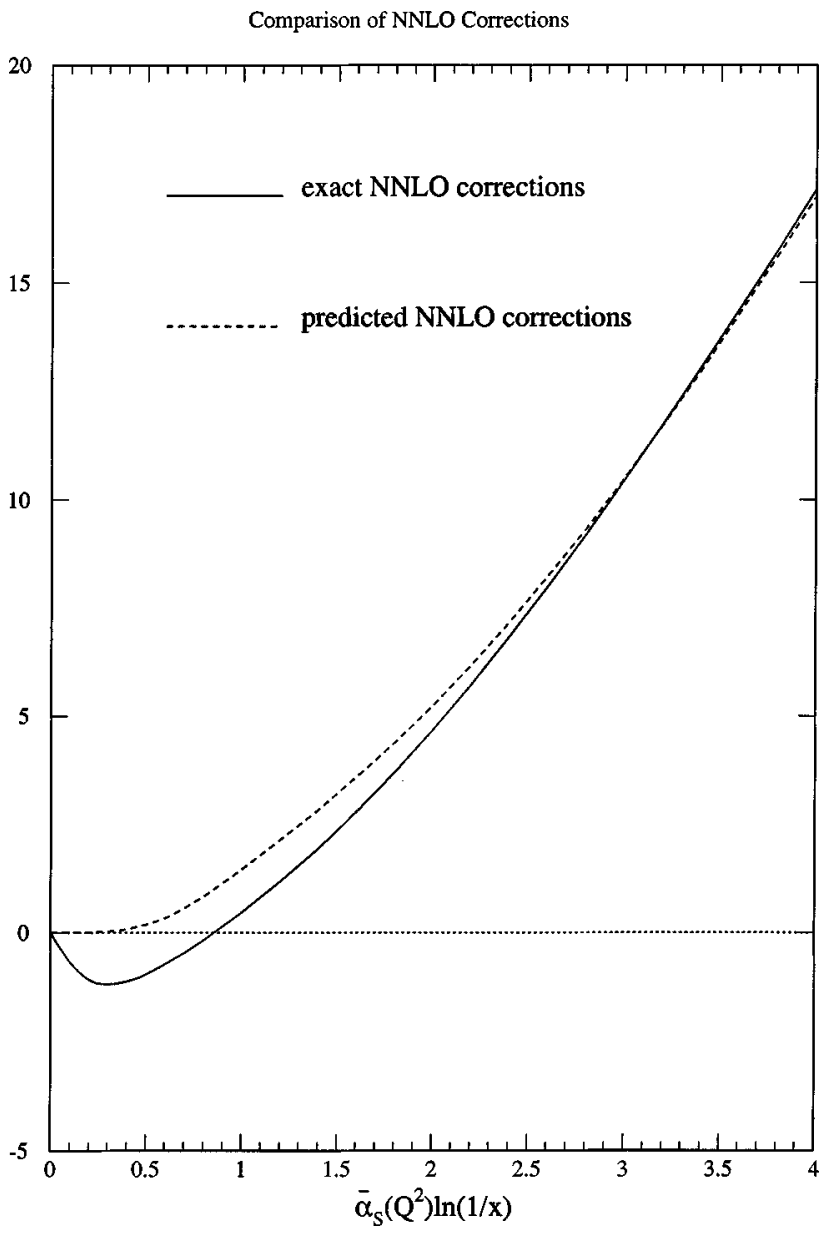

FIG. 4. Comparison of the exact NNLO splitting function $\hat{p}^{2}\left(\bar{\alpha}_{s}\left(Q^{2}\right) \xi\right)$ with the value predicted from the choice of scale in the LO expression. Both terms are weighted by $\left(\exp \left[\lambda\left(Q^{2}\right) \xi\right]\right)^{-1}$.

the $\mathcal{O}\left(\left[\beta_{0} \alpha_{s}\left(Q^{2}\right)\right]^{2}\right)$ contribution to the splitting function exactly, and compare this to that expected if the scale choice is correct, i.e.,

$$
\begin{gathered}
\frac{1}{2}\left(\beta_{0} \alpha_{s}\left(Q^{2}\right)\right)^{2}\left(\frac{\partial^{2} p^{0}\left(x, \bar{\alpha}_{s}\left(Q^{2}\right)\right)}{\partial\left(\ln \alpha_{s}\left(Q^{2}\right)\right)^{2}}+2 \frac{\partial p^{0}\left(x, \bar{\alpha}_{s}\left(Q^{2}\right)\right)}{\partial\left(\ln \alpha_{s}\left(Q^{2}\right)\right)}\right) \\
\quad \times\left(\frac{\hat{p}^{1}\left(x, \bar{\alpha}_{s}\left(Q^{2}\right)\right)}{\partial p^{0}\left(x, \bar{\alpha}_{s}\left(Q^{2}\right)\right) / \partial\left(\ln \alpha_{s}\left(Q^{2}\right)\right)}\right)^{2} .
\end{gathered}
$$

The relevant terms in $a\left(N, Q^{2}\right)$ can be found by considering the terms in Eq. (4.4) multiplying the Gaussian which go like $\gamma^{6} /\left(\bar{\beta}_{0} N\right)^{2}$ and $\gamma^{4} /\left(\bar{\beta}_{0} N\right)$, performing the Gaussian integrals and using the equality $N=\bar{\alpha}_{s}\left(Q^{2}\right) \gamma^{0}$. This gives

$$
\begin{aligned}
a\left(Q^{2}, N\right)= & a^{S P}\left(Q^{2}, N\right)\left(1-\beta_{0} \alpha_{s}\left(Q^{2}\right)\left[\frac{5\left(\chi^{\prime \prime}\left(\gamma^{0}\right)\right)^{2} \chi\left(\gamma^{0}\right)}{24\left(-\chi^{\prime}\left(\gamma^{0}\right)\right)^{3}}\right.\right. \\
& \left.\left.-\left(\frac{\chi^{\prime \prime}\left(\gamma^{0}\right)}{2 \gamma^{0}}-\frac{\chi^{\prime \prime \prime}\left(\gamma^{0}\right)}{8}\right) \frac{\chi\left(\gamma^{0}\right)}{\left(-\chi^{\prime}\left(\gamma^{0}\right)\right)^{2}}\right]+\cdots\right) .
\end{aligned}
$$

Inserting into Eq. (4.6) and making the transformation to $x$ space we obtain the required $\mathcal{O}\left(\left[\beta_{0} \alpha_{s}\left(Q^{2}\right)\right]^{2}\right)$ splitting function $\hat{p}^{2}\left(\bar{\alpha}_{s}\left(Q^{2}\right) \xi\right)$. This is compared to Eq. (4.26) in Fig. 4, where each term is weighted by $\left(\exp \left[\lambda\left(Q^{2}\right) \xi\right]\right)^{-1}$, and the upper limit of $\bar{\alpha}_{s}\left(Q^{2}\right) \xi=4$ is chosen since the first 20 terms in the series expansions of each expression give a very accurate representation up to this value and it easily covers the range relevant for comparison to HERA data. As one can see, above $\bar{\alpha}_{s}\left(Q^{2}\right) \xi=1$ the ansatz for the $\mathcal{O}\left(\left[\beta_{0} \alpha_{s}\left(Q^{2}\right)\right]^{2}\right)$ contribution of the splitting function matches extremely well to the explicitly calculated value. Below $\bar{\alpha}_{s}\left(Q^{2}\right) \xi=1$ the matching is not so good, but this is relatively unimportant since in this region this contribution to the total splitting function is small compared to the more leading contributions, i.e., the scale change is quite small and $p^{0}\left(\bar{\alpha}_{s}\left(Q^{2}\right) \xi\right)$ $\gg \beta_{0} \alpha_{s}\left(Q^{2}\right) \hat{p}^{1}\left(\bar{\alpha}_{s}\left(Q^{2}\right) \xi\right) \gg\left(\beta_{0} \alpha_{s}\left(Q^{2}\right)\right)^{2} \hat{p}^{2}\left(\bar{\alpha}_{s}\left(Q^{2}\right) \xi\right)$. In this region the scale choice is also sensitive to the interference with the finite $x$ effects at fixed order in $\alpha_{s}\left(Q^{2}\right)$ which are ignored using this expansion scheme. Hence, it seems reasonable to conclude that explicit checks strongly support the assumption that all the running coupling effects in the evolution can be accurately described by the use of the effective scale obtained by solving Eq. (4.12) in the LO effective splitting function.

\section{SOLVING THE BFKL EQUATION FOR RUNNING COUPLING: INPUT}

We could also attempt to evaluate $b\left(Q_{0}^{2}, N\right)$ in the same manner, i.e., expanding about $\gamma^{0}\left(\bar{\alpha}_{s}\left(Q_{0}^{2}\right) / N\right)$ and calculating an order by order series in $\beta_{0} \alpha_{s}\left(Q_{0}^{2}\right)$. Of course, without an infrared regulator we know that $b\left(Q_{0}^{2}, N\right)$ must be divergent because the integrand has singularities along the contour of integration, i.e., at integer values of $\tilde{\gamma}$, which lead to ambiguities of order $\left(\Lambda^{2} / Q_{0}^{2}\right)^{n}$, i.e., higher twist. These singularities do indeed show up in this power series solution. Expanding about $\gamma^{0}\left(\alpha_{s}\left(Q_{0}^{2}\right) / N\right)$ and only keeping the lowest order terms one obtains a sensible solution, i.e.,

$$
\begin{aligned}
b^{S P}\left(Q_{0}^{2}, N\right)= & \frac{1}{\left(\bar{\alpha}_{s}\left(Q_{0}^{2}\right) / N\right)\left[-\chi^{\prime}\left(\gamma^{0}\left[\bar{\alpha}_{s}\left(Q_{0}^{2}\right) / N\right]\right)\right]^{1 / 2}} \\
& \times \exp \left(\int-Q_{0}^{2} \gamma^{0}\left(\bar{\alpha}_{s}\left(q^{2}\right) / N\right) d \ln q^{2}\right) .
\end{aligned}
$$

Going beyond this approximation one obtains the same sort of series as for $a\left(N, Q^{2}\right)$, except that because the contour is now along the real axis, rather than parallel to the imaginary axis, the terms in the series are all of the same sign rather than oscillating. This leads to at least one power series behavior of the form

$$
\sum_{n=0}^{\infty} B_{n} n !\left(\beta_{0} \alpha_{s}\left(Q_{0}^{2}\right)\right)^{n}\left(\alpha_{s}\left(Q_{0}^{2}\right) /\left[N-\lambda\left(Q_{0}^{2}\right)\right]\right)^{n / 2},
$$


where $B_{n}$ is roughly $B^{n}$, and $B \sim 3.6$. One can take the inverse transformation of this series term by term, obtaining a power series in $x$ space which sums to approximately the form

$$
\exp \left(\lambda\left(Q_{0}^{2}\right) \xi\right) \sum_{n=0}^{\infty}\left(B \beta_{0} \alpha_{s}^{3 / 2}\left(Q_{0}^{2}\right) \xi^{1 / 2}\right)^{n}
$$

Hence, in this case the power series is suggestive of the fact that due to the diffusion into the infrared the appropriate coupling for $b\left(Q_{0}^{2}, N\right)$ depends not simply on $\ln \left(Q_{0}^{2} / \Lambda^{2}\right)$ but on $\ln \left(Q_{0}^{2} / \Lambda^{2}\right)-3.63 \beta_{0}\left(\alpha_{s}\left(Q_{0}^{2}\right) \xi\right)^{1 / 2}$, the exact opposite of the case for $a\left(Q^{2}, N\right)$.

Of course, the infrared diffusion is a rather complicated problem, and the series in Eq. (5.3) is only convergent for $\left(B \beta_{0} \alpha_{s}^{3 / 2}\left(Q_{0}^{2}\right) \xi^{1 / 2}\right) \leqslant 1$. This indicates that $\mathrm{I}$ have been too simplistic in transforming Eq. (5.2) to $x$ space term by term. The series in Eq. (5.2) is not defined for any $N$, and before going to $x$ space we must solve this problem. The series (5.2) may be summed using standard Borel transformation techniques. This leads to a well-defined series up to an ambiguity of the form $\exp \left(-\left[N-\lambda\left(Q_{0}^{2}\right)\right]^{1 / 2} /\left[B \beta_{0} \alpha_{s}^{3 / 2}\left(Q_{0}^{2}\right)\right]\right)$. Now performing the transformation to $x$ space we obtain a wellbehaved series in $\left(B \beta_{0} \alpha_{s}^{3 / 2}\left(Q_{0}^{2}\right) \xi^{1 / 2}\right)$ as well as an ambiguity of order $\exp \left(\lambda\left(Q_{0}^{2}\right) \xi\right) \exp \left(-1 /\left[B^{2} \beta_{0}^{2} \alpha_{s}^{3}\left(Q_{0}^{2}\right) \xi\right]\right)$, where $B^{2}$ $\approx 13$. This latter ambiguity is due to the presence of an infrared renormalon [34] in the expression for $b\left(Q_{0}^{2}, N\right)$, and will be cancelled by similar ambiguities in higher twist corrections. ${ }^{12}$ Such terms are therefore taken to estimate the size of higher twist effects. In this case we see that due to diffusion becoming enhanced at small $x$, this infrared induced uncertainty quickly becomes large at small $x$, and indeed the calculation of the normalization of the gluon Green's function is only at all reliable in the limit

$$
13 \beta_{0}^{2} \alpha_{s}^{3}\left(Q_{0}^{2}\right) \xi \ll 1 \text {. }
$$

Hence, we find that even if we had a reliable model for the bare gluon distribution $g_{B}\left(Q_{0}^{2}, N\right)^{13}$ we cannot calculate the input for the gluon distribution at small $x$ within perturbation theory, and previous conclusions on the infrared diffusion physics ruining perturbative predictability $[23,7]$ are confirmed. In particular we note that the requirement in Eq. (5.4) is basically identical to that found in [23,7], and indeed, if the series in Eq. (45) of the latter is summed it has an ambiguity of exactly the same type as discussed above (though in [7] the series in $x$ space was found directly). However, here I stress that this ambiguity is unique to the normalization function, and does not affect the evolution, which is calculable in perturbation theory.

\footnotetext{
${ }^{12}$ The ambiguity is seen as the nonperturbative contribution to the solution in [9].

${ }^{13}$ Given that the function $b\left(Q_{0}^{2}, N\right)$ is dependent on the type of collinear regularization as well as the ambiguity discussed above this actually seems rather unlikely.
}

Before finishing this section it is interesting to discuss the relationship between the solutions obtained via the techniques in this paper and solutions obtained by other authors. It has been noted by several authors [7-9] that the asymptotic solution for the BFKL equation with running coupling has the general form

$$
\begin{aligned}
f\left(Q^{2}, Q_{0}^{2}, \xi\right) \sim & \frac{1}{\left(\alpha_{s} \xi\right)^{1 / 2}} \exp \left(\lambda\left(Q Q_{0}\right) \xi+K \beta_{0}^{2} \alpha_{s}^{5} \xi^{3}\right. \\
& \left.-\frac{\ln ^{2}\left(Q^{2} / Q_{0}^{2}\right)}{56 \zeta(3) \bar{\alpha}_{s} \xi}+\cdots\right)
\end{aligned}
$$

where unless explicitly stated $\alpha_{s}$ is at some fixed scale $\mu$, and $K=(7 / 6) \zeta(3)(3 / \pi)^{3}(4 \ln 2)^{2}$. This seems rather at odds with the results discussed above. However, it is not difficult to see how this solution appears. Ignoring the term in the exponent going like $\alpha_{s}^{5} \xi^{3}$ one achieves a solution of this form simply by taking the transformation to $x$ space of the product $a^{S P}\left(Q^{2}, N\right) b^{S P}\left(Q_{0}^{2}, N\right)$ in the limit $x \rightarrow 0$, and only keeping the most dominant terms in the series expansions of the couplings about scale $\mu$.

It is not too much extra work to see where the $\alpha_{s}^{5} \xi^{3}$ terms come from. Consider if rather than taking the saddle point approximations for $a\left(Q^{2}, N\right)$ and $b\left(Q_{0}^{2}, N\right)$ one takes the solution of Eq. (4.22) for $a\left(Q^{2}, N\right)$ and the equivalent expansion for $b\left(Q_{0}^{2}, N\right)$. The solution for $b\left(Q_{0}^{2}, N\right)$ in this approximation is of precisely the same form as Eq. (4.23) once we replace $a^{S P}\left(Q^{2}, N\right)$ with $b^{S P}\left(Q_{0}^{2}, N\right)$ and remove the factors of $(-1)^{n}$ (the $L_{n}$ are identical). If we multiply the two series in these expressions together then since at large orders $L_{n}$ $\sim a^{n} n$ !, the resulting series is to good accuracy proportional to

$$
\sum_{n=0}^{\infty} L_{2 n}\left(\left(\beta_{0} \alpha_{s}\right)^{2}[\lambda /(N-\lambda)]^{3}\right)^{n}
$$

where $\alpha_{s} \equiv \alpha_{s}\left(\mu^{2}\right)$, i.e., we expand $\alpha_{s}\left(Q^{2}\left(Q_{0}^{2}\right)\right)$ about $\alpha_{s}\left(\mu^{2}\right), \quad$ and asymptotically $L_{2 n+2} / L_{2 n} \rightarrow(63 \zeta(3) /$ $(8 \ln 2)) n^{2}$. Multiplying this by the two saddle point solutions, and performing the transformation to $x$ space this sum introduces precisely $\exp \left(K \beta_{0}^{2} \alpha_{s}^{5} \xi^{3}\right)$ with the correct value of $K$. Hence, this non-Regge term comes about due to interference between the input term $b\left(Q_{0}^{2}, N\right)$ and the evolution term $a\left(Q^{2}, N\right)$.

Hence, these previous results do appear by taking the transformation to $x$ space of the product of truncated solutions for $a\left(Q^{2}, N\right)$ and $b\left(Q_{0}^{2}, N\right)$. However, I would argue that these solutions are not representative of any real physics, since neither of these truncations is at all accurate except at quite high $x$. For given $Q_{0}^{2}$ Eq. (5.5) is only applicable for $x$ satisfying Eq. (5.4), in which case the $x \rightarrow 0$ approximations used to derive Eq. (5.5) are generally rather inaccurate. When Eq. (5.4) is not satisfied the transformation of $b\left(Q_{0}^{2}, N\right)$ is indeterminate, and that of $a\left(Q^{2}, N\right)$ requires resummation. The only sensible option seems to be to factor out $b\left(Q_{0}^{2}, N\right)$ and simply use $a\left(Q^{2}, N\right)$ to determine the evolution as accurately as possible, rather than trying to find 
$f\left(Q^{2}, Q_{0}^{2}, \xi\right)$. Then we know from the general arguments already discussed that the Regge term $\exp (\lambda \xi)$ is nothing to do with the true result, let alone the non-Regge term $\exp \left(K \beta_{0}^{2} \alpha_{s}^{5} \xi^{3}\right)$.

\section{NLO CORRECTIONS TO THE BFKL EQUATION}

So far I have simply assumed that an accurate way to account for the running of the coupling in the LO BFKL equation is to use Eq. (3.1). This is an assumption which involves the resummation of an infinite number of terms, i.e., it assumes that at all orders in $\alpha_{s}\left(\mu^{2}\right)$ the dominant contribution to the BFKL equation due to the running coupling is

$$
\frac{\bar{\alpha}_{s}}{N}(-1)^{n}\left(\beta_{0} \alpha_{s}\left(\mu^{2}\right) \ln \left(k^{2} / \mu^{2}\right)\right)^{n} \int_{0}^{\infty} \frac{d q^{2}}{q^{2}} K^{0}\left(q^{2}, k^{2}\right) f\left(q^{2}\right) .
$$

Until recently this has been an assumption for all $n \geqslant 1$ although the above terms must be present. However, the recent calculation of the NLO correction to the BFKL equation has given us some insight into this question. Formally the NLO BFKL equation may be written as

$$
\begin{aligned}
f\left(k^{2}, Q_{0}^{2}, \bar{\alpha}_{s}\left(\mu^{2}\right) / N\right)= & f^{0}\left(k^{2}, Q_{0}^{2}\right) \\
& +\left(\frac{\bar{\alpha}_{s}\left(\mu^{2}\right)}{N}\right) \int_{0}^{\infty} \frac{d q^{2}}{q^{2}}\left(K^{0}\left(q^{2}, k^{2}\right)\right. \\
& -\beta_{0} \alpha_{s}\left(\mu^{2}\right) \ln \left(k^{2} / \mu^{2}\right) K^{0}\left(q^{2}, k^{2}\right) \\
& \left.-\alpha_{s}\left(\mu^{2}\right) K^{1}\left(q^{2}, k^{2}\right)\right) f\left(q^{2}\right),
\end{aligned}
$$

where $K^{1}\left(q^{2}, k^{2}\right)$ can be found in [2]. This is the strictly NLO equation with no resummation at all. The separation of the NLO part into the running coupling part and the part depending on $K^{1}\left(q^{2}, k^{2}\right)$ is arbitrary. The former is the first term in the infinite series we have already considered, but the latter also contains some pieces which may be associated with the running of the coupling, i.e., going like $\beta_{0}$.

This equation can be solved using the same methods which were applied in Sec. IV. Taking the Mellin transformation, this time with respect to $\left(k^{2} / \mu^{2}\right)$ we obtain

$$
\begin{aligned}
\widetilde{f}(\gamma, N)= & \widetilde{f}^{0}\left(\gamma, Q_{0}^{2}\right)+\left(\frac{\bar{\alpha}_{s}\left(\mu^{2}\right)}{N}\right) \\
& \times\left(\left(\chi_{0}(\gamma)-\alpha_{s}\left(\mu^{2}\right) \chi_{1}(\gamma)\right) \tilde{f}(\gamma, N)\right. \\
& \left.+\beta_{0} \alpha_{s}\left(\mu^{2}\right) \frac{d\left(\chi_{0}(\gamma) \tilde{f}(\gamma)\right)}{d \gamma}\right),
\end{aligned}
$$

where $\chi^{1}(\gamma)$ can also be found in [2]. As in Sec. III, this is a first order differential equation in $\gamma$, and it can be solved in the same manner. In fact it is rather easier to alter Eq. (6.2) to the slightly different form

$$
\begin{aligned}
f\left(k^{2}, Q_{0}^{2}, \mu^{2}\right)= & f^{0}\left(k^{2}, Q_{0}^{2}\right)+\left(\frac{\bar{\alpha}_{s}\left(k^{2}\right)}{N}\right) \int_{0}^{\infty} \frac{d q^{2}}{q^{2}}\left(K^{0}\left(q^{2}, k^{2}\right)\right. \\
& \left.-\alpha_{s}\left(\mu^{2}\right) K^{1}\left(q^{2}, k^{2}\right)\right) f\left(q^{2}\right) .
\end{aligned}
$$

This is identical to Eq. (6.2) up to NNLO in $\alpha_{s}\left(\mu^{2}\right)$ and is a common way for the NLO BFKL equation to be written since it makes the solution easier. One must simply remember that the solution obtained is only uniquely defined up to NLO in $\alpha_{s}\left(\mu^{2}\right)$ when the coupling $\alpha_{s}\left(k^{2}\right)$ is expanded about $\alpha_{s}\left(\mu^{2}\right)$. If we take the Mellin transformation of Eq. (6.4) with respect to $\left(k^{2} / \Lambda^{2}\right)$ we obtain

$$
\begin{aligned}
\frac{d \tilde{f}(\gamma, N)}{d \gamma}= & \frac{d \tilde{f}^{0}\left(\gamma, Q_{0}^{2}\right)}{d \gamma}-\frac{1}{\widetilde{\beta}_{0} N}\left(\chi_{0}(\gamma)\right. \\
& \left.-\alpha_{s}\left(\mu^{2}\right) \chi_{1}(\gamma)\right) \tilde{f}(\gamma, N),
\end{aligned}
$$

which is identical to Eq. (3.3) except for the NLO in $\alpha_{s}\left(\mu^{2}\right)$ correction to the kernel. It can therefore be solved in exactly the same manner as this previous equation [this would also be true for Eq. (6.3)], again obtaining a solution factorizing into a $Q^{2}$-dependent part and a $Q_{0}^{2}$ dependent part. Each of these is a contour integral and analogously to the previous treatment expanding about the saddle point when performing the inverse Mellin transformation to $Q^{2}$ or $Q_{0}^{2}$ space produces an ordered series in $\alpha_{s}\left(\mu^{2}\right)$, as long as we also expand $\alpha_{s}\left(Q^{2}\left(Q_{0}^{2}\right)\right)$ about $\alpha_{s}\left(\mu^{2}\right)$. This time the saddle point is at [35]

$$
\begin{aligned}
\gamma^{\mathrm{NLO}, S P}\left(\bar{\alpha}_{s} / N\right)= & \gamma^{0}\left(\bar{\alpha}_{s} / N\right) \\
& -\beta_{0} \alpha_{s} \ln \left(Q^{2}\left(Q_{0}^{2}\right) / \mu^{2}\right) \frac{\partial \gamma^{0}\left(\bar{\alpha}_{s} / N\right)}{\partial\left(\ln \left(\alpha_{s}\right)\right)} \\
& -\alpha_{s} \frac{\chi_{1}\left(\gamma^{0}\left(\bar{\alpha}_{s} / N\right)\right)}{-\chi_{0}^{\prime}\left(\gamma^{0}\left(\bar{\alpha}_{s} / N\right)\right)}+\cdots,
\end{aligned}
$$

where

$$
\alpha_{s} \frac{\chi_{1}\left(\gamma^{0}\left(\bar{\alpha}_{s} / N\right)\right)}{-\chi_{0}^{\prime}\left(\gamma^{0}\left(\bar{\alpha}_{s} / N\right)\right)} \equiv \alpha_{s} \gamma^{1}\left(\bar{\alpha}_{s} / N\right)
$$

is often called the NLO-BFKL anomalous dimension, and all other corrections are beyond NLO in $\alpha_{s}{ }^{14}$

Using the previous choice of input we can evaluate the two inverse transformations about the saddle point [we only need go further than the strict saddle point approximation when considering the $(1 / \gamma)$ factor in the $Q^{2}$-dependent integrand to obtain all results up to NLO accuracy-i.e., we use $\left.1 /\left(\gamma^{0}+\gamma\right)^{-1}=1 / \gamma^{0}-\gamma /\left(\gamma^{0}\right)^{2}\right]$. This gives a solution for the gluon structure function of the form

\footnotetext{
${ }^{14}$ For the remainder of this section unless the argument is explicitly stated $\alpha_{s} \equiv \alpha_{s}\left(\mu^{2}\right)$.
} 


$$
\begin{aligned}
\frac{\mathcal{G}\left(Q^{2}, N\right)}{g_{B}\left(Q_{0}^{2}, N\right)}= & \frac{1}{\left(\bar{\alpha}_{s} / N\right) \gamma^{0}\left(-\chi_{0}^{\prime}\left(\gamma^{0}\right)\right)}\left(1-\alpha_{s}\left(-\frac{\gamma^{1}}{\gamma^{0}}+\gamma^{1} \frac{\chi_{0}^{\prime \prime}\left(\gamma^{0}\right)}{-\chi_{0}^{\prime}\left(\gamma^{0}\right)}+\frac{\chi_{1}^{\prime}\left(\gamma^{0}\right)}{-\chi_{0}^{\prime}\left(\gamma^{0}\right)}-\beta_{0} \frac{\chi_{0}^{\prime \prime}\left(\gamma^{0}\right) \chi_{0}\left(\gamma^{0}\right)}{2 \gamma^{0}\left(-\chi_{0}^{\prime}\left(\gamma^{0}\right)\right)^{2}}\right)\right. \\
& \left.-\beta_{0} \alpha_{s} \ln \left(Q^{2} / \mu^{2}\right) \frac{\partial \bar{\gamma}^{0}}{\partial \ln \left(\alpha_{s}\right)}\left(-\frac{1}{\gamma^{0}}+\frac{1}{2} \frac{\chi_{0}^{\prime \prime}\left(\gamma^{0}\right)}{-\chi_{0}^{\prime}\left(\gamma^{0}\right)}\right)-\beta_{0} \alpha_{s} \ln \left(Q_{0}^{2} / \mu^{2}\right)\left(-1+\frac{1}{2} \frac{\partial \gamma^{0}}{\partial \ln \left(\alpha_{s}\right)} \frac{\chi_{0}^{\prime \prime}\left(\gamma^{0}\right)}{-\chi_{0}^{\prime}\left(\gamma^{0}\right)}\right)\right) \\
& \times \exp \left(\int_{Q_{0}^{2}}^{Q^{2}}\left(\gamma^{0}-\beta_{0} \alpha_{s} \ln \left(q^{2} / \mu^{2}\right) \frac{\partial \gamma^{0}}{\partial\left(\ln \left(\alpha_{s}\right)\right)}-\alpha_{s} \gamma^{1}\right) d \ln q^{2}\right) .
\end{aligned}
$$

This allows us to determine the gluon coefficient function and gluon anomalous dimension up to NLO in $\alpha_{s}\left(\mu^{2}\right)$, where the former may be defined as the value of Eq. (6.8) when $Q_{0}^{2}=Q^{2}$, and the latter is then determined by the evolution of Eq. (6.8) with respect to $Q^{2}$ once the coefficient function has been subtracted out, i.e.,

$$
\begin{aligned}
\mathcal{R}^{\mathrm{NLO}}\left(\bar{\alpha}_{s} / N, Q^{2} / \mu^{2}\right)= & \frac{1}{\left(\bar{\alpha}_{s} / N\right) \gamma^{0}\left(-\chi_{0}^{\prime}\left(\gamma^{0}\right)\right)}\left[1-\alpha_{s}\left(-\frac{\gamma^{1}}{\gamma^{0}}+\gamma^{1} \frac{\chi_{0}^{\prime \prime}\left(\gamma^{0}\right)}{-\chi_{0}^{\prime}\left(\gamma^{0}\right)}+\frac{\chi_{1}^{\prime}\left(\gamma^{0}\right)}{-\chi_{0}^{\prime}\left(\gamma^{0}\right)}\right)\right. \\
& \left.+\alpha_{s} \beta_{0}\left(\frac{\chi_{0}^{\prime \prime}\left(\gamma^{0}\right) \chi_{0}\left(\gamma^{0}\right)}{2 \gamma^{0}\left(-\chi_{0}^{\prime}\left(\gamma^{0}\right)\right)^{2}}-\ln \left(Q^{2} / \mu^{2}\right)\left[-1+\frac{\partial \gamma^{0}}{\partial \ln \left(\alpha_{s}\right)}\left(-\frac{1}{\gamma^{0}}+\frac{\chi_{0}^{\prime \prime}\left(\gamma^{0}\right)}{-\chi_{0}^{\prime}\left(\gamma^{0}\right)}\right)\right]\right)\right]
\end{aligned}
$$

and

$$
\gamma^{\mathrm{NLO}}\left(\bar{\alpha}_{s} / N, Q^{2} / \mu^{2}\right)=\gamma^{0}-\beta_{0} \alpha_{s} \ln \left(Q^{2} / \mu^{2}\right) \frac{\partial \gamma^{0}}{\partial\left(\ln \left(\alpha_{s}\right)\right)}-\alpha_{s} \gamma^{1}+\left(-1+\frac{1}{2} \frac{\partial \gamma^{0}}{\partial \ln \left(\alpha_{s}\right)} \frac{\chi_{0}^{\prime \prime}\left(\gamma^{0}\right)}{-\chi_{0}^{\prime}\left(\gamma^{0}\right)}\right)
$$

It is gratifying, though necessary, that in both cases the LO results from Eq. (2.15) are reproduced, and the terms $\sim \beta_{0} \alpha_{s} \ln \left(Q^{2} / \mu^{2}\right)$ are consistent with the renormalization group. [Note that $-\alpha_{s} \gamma^{1}\left(\bar{\alpha}_{s} / N\right)$ is not really the NLO correction to the anomalous dimension in this scheme-it is actually quite similar to the modified minimal subtraction $(\overline{\mathrm{MS}})$ factorization scheme anomalous dimension.] Nevertheless, both of these quantities are dependent on our choice of input and factorization scheme, and do not contain any real physics.

The only physically unambiguous quantity which may be extracted is the effective anomalous dimension defined by Eq. (4.6):

$$
\begin{aligned}
\Gamma\left(N, Q^{2} / \mu^{2}\right)= & \gamma^{0}-\beta_{0} \alpha_{s}\left(\frac{\partial \gamma^{0}}{\partial \ln \left(\alpha_{s}\right)} \ln \left(Q^{2} / \mu^{2}\right)\right. \\
& \left.+\frac{\partial \gamma^{0}}{\partial \ln \left(\alpha_{s}\right)}\left(\frac{-\chi^{\prime \prime}\left(\gamma^{0}\right)}{2 \chi^{\prime}\left(\gamma^{0}\right)}-\frac{1}{\gamma^{0}}\right)\right)-\alpha_{s} \gamma^{1} .
\end{aligned}
$$

The second term on the right corresponds to the NLO in $\alpha_{s}$ contributions previously accounted for when considering the running coupling, while the third gives the additional NLO corrections. By examining the part of $\gamma^{1}$ which depends on $\beta_{0}$ we can check whether at NLO at least the previous assumption about the manner in which to treat running coupling effects was correct, i.e., can see whether these do give the dominant contribution at NLO, or whether the conformal parts of $\gamma^{1}$ are more important.

One can study the terms in Eq. (6.11) by finding the explicit form of each as a power series in $\bar{\alpha}_{s} / N$. However, in the small $x$ limit we can examine the form of the singularities in the $N$ plane, i.e., the limit of each of the terms as $\gamma^{0} \rightarrow \frac{1}{2}$ and $N \rightarrow \lambda$. Using the well publicized fact that $\chi_{1}\left(\frac{1}{2}\right)$ $=4 \ln 2 \times 6.3$ for 4 flavors and in the $\overline{\mathrm{MS}}$ renormalization scheme, and taking the inverse transformation back to $x$ space of Eq. (6.11), we obtain

$$
\begin{aligned}
x P\left(x, Q^{2}\right)= & \bar{\alpha}_{s} \exp (\lambda \xi)\left(\frac{0.068}{\left(\bar{\alpha}_{s} \xi\right)^{3 / 2}}\right. \\
& -\beta_{0} \alpha_{s}\left[\left(\frac{0.188}{\left(\bar{\alpha}_{s} \xi\right)^{1 / 2}}\right) \ln \left(Q^{2} / \mu^{2}\right)+0.69\right] \\
& \left.-\alpha_{s}\left(\frac{1.18}{\left(\bar{\alpha}_{s} \xi\right)^{1 / 2}}\right)\right) .
\end{aligned}
$$

Hence, the last term, although numerically large, is subleading to the effects due to the running of the coupling we have previously considered, being a power of $\left(\bar{\alpha}_{s} \xi\right)^{1 / 2}$ smaller. However, now we can be a little more systematic. Examining the full NLO correction $\chi_{1}(\gamma)$, presented in Eq. (14) in [2], we see that there are contributions which may be interpreted as being due to the running of the coupling. These are $\frac{1}{2} \beta_{0}\left(\chi^{2}(\gamma)+\chi^{\prime}(\gamma)\right)$ and $-(5 / 3) \beta_{0} \chi(\gamma)$, coming from the 
NLO correction to the Reggeon-Reggeon-gluon vertex and the purely virtual terms, respectively. ${ }^{15}$ We imagine that these should be moved out of $\gamma^{1}$ in Eq. (6.11) and put into the $\beta_{0}$-dependent part of the NLO correction. Doing this changes Eq. (6.12) to

$$
\begin{aligned}
x P\left(x, Q^{2}\right)= & \bar{\alpha}_{s} \exp (\lambda \xi)\left(\frac{0.068}{\left(\bar{\alpha}_{s} \xi\right)^{3 / 2}}\right. \\
& -\beta_{0} \alpha_{s}\left[\left(\frac{0.188 \ln \left(Q^{2} / \mu^{2}\right)-0.05}{\left(\bar{\alpha}_{s} \xi\right)^{1 / 2}}\right)+0.69\right] \\
& \left.-\alpha_{s}\left(\frac{1.23}{\left(\bar{\alpha}_{s} \xi\right)^{1 / 2}}\right)\right)
\end{aligned}
$$

Therefore, not only is this additional NLO correction due to the running of the coupling numerically very small, but it is also subleading at small $x$ to the terms we have already considered. ${ }^{16}$ Choosing the renormalization scale $\mu$ by setting the $\beta_{0}$ dependent term to zero, ${ }^{17}$ we obtain a very minor correction to our previous choice of scale for the limit $x$ $\rightarrow 0$, i.e.,

$\ln \left(Q^{2} / \Lambda^{2}\right) \rightarrow \ln \left(Q^{2} / \Lambda^{2}\right)-0.26+\frac{(56 \zeta(3) \pi)^{1 / 2}}{4}\left(\bar{\alpha}_{s}\left(Q^{2}\right) \xi\right)^{1 / 2}$,

where in fact there should really be an additional constant on the right in the above equation due to subleading corrections in Eq. (4.21) that I have ignored. The constant on the right of Eq. (6.14) is also renormalization scheme dependent, though the dominant $3.63\left(\bar{\alpha}_{s}\left(Q^{2}\right) \xi\right)^{1 / 2}$ term is not.

We can also solve the equation for the scale exactly rather than in the small $x$ limit. Putting our additional terms into the definition of the running coupling dependent NLO splitting function, we compare with the previous $\hat{p}^{1}\left(\bar{\alpha}_{s}\left(Q^{2}\right) \xi\right)$ in Fig. 5 . We see that indeed the corrected $\hat{p}^{1}\left(\bar{\alpha}_{s}\left(Q^{2}\right) \xi\right)$ is slightly smaller than the original for $\bar{\alpha}_{s}\left(Q^{2}\right) \xi \geqslant 1$ but is different at higher $x$, implying a different scale choice here to that in Sec. IV. Of course, at these higher values of $x$ the differences are not too important since, as already mentioned, the scale changes are small here, and there will be interference with other effects from the order by order in $\alpha_{s}$ expansion.

Hence, we find that at NLO our previous assumption about the $-\beta_{0} \alpha_{s}\left(\mu^{2}\right) \ln \left(k^{2} / \mu^{2}\right)$ term (which had to be present) being the dominant contribution associated with the

\footnotetext{
${ }^{15}$ It does not seem certain whether or not the second of these terms should be included as a running coupling effect or not. As will become clear below this is only relevant for the scale choice at high $x$ where other considerations from large $x$ terms come into play also.

${ }^{16}$ Not including the $-(5 / 3) \beta_{0} \chi(\gamma)$ term would simply lead to -0.05 becoming 0.26 and 1.23 subsequently becoming 0.92 .

${ }^{17}$ I choose $\alpha_{s}$ to be $\alpha_{s}\left(Q^{2}\right)$ rather than $\alpha_{s}\left(\mu^{2}\right)$ when doing this. The two are of course equivalent up to higher order corrections, but the results of previous sections suggest that this is the appropriate choice.
}

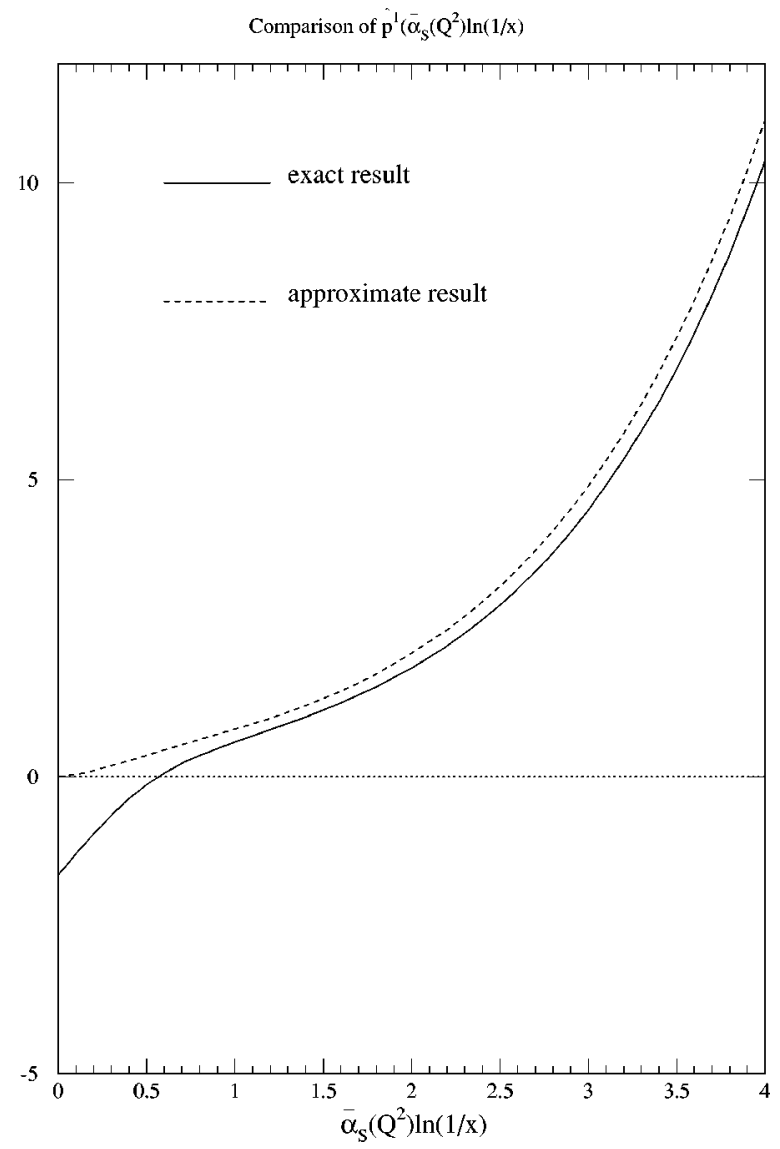

FIG. 5. Comparison of the exact NLO $\beta_{0}$-dependent splitting function $\hat{p}^{1}\left(\bar{\alpha}_{s}\left(Q^{2}\right) \xi\right)$ including the corrections from $\gamma^{1}$ with the value of $\hat{p}^{1}\left(\bar{\alpha}_{s}\left(Q^{2}\right) \xi\right)$ obtained using the assumption in Sec. IV. Both terms are weighted by $\left(\exp \left[2 \alpha_{s}\left(Q^{2}\right) \xi\right]\right)^{-1}$ for ease of comparison.

running coupling is very well justified. This gives us confidence, if not a proof, that the approach taken in the previous sections, i.e., that the $\left[-\beta_{0} \alpha_{s}\left(\mu^{2}\right) \ln \left(k^{2} / \mu^{2}\right)\right]^{n}$ terms are the dominant contribution from the running of the coupling at all orders is roughly correct. Consequently, this full NLO result also supports the hypothesis that the LO running coupling effects can be taken account of simply by using the $x$-dependent scale choice, determined by the BLM prescription, in the LO expression for the effective splitting function.

Before considering the details of the NLO corrections to the kernel which are not associated with the running of the coupling let us reconsider the NLO BFKL equation. Given the above results it seems very unlikely that the NLO BFKL equation as written in Eq. (6.4) is will be a good representation of the real physics since the overall power of the coupling is allowed to run with $k^{2}$ while that associated with the NLO kernel is fixed at $\mu^{2}$. Bearing in mind that letting the coupling run in the LO equation leads to such dramatic effects, and that at higher orders there will definitely be the logs in $\left(k^{2} / \mu^{2}\right)$ associated with the running of this additional factor of $\alpha_{s}\left(\mu^{2}\right)$ (with what now seem likely to be small corrections) it seems most appropriate to write the NLO BFKL equation with running coupling as 


$$
\begin{aligned}
f\left(k^{2}, Q_{0}^{2}, \mu^{2}\right)= & f^{0}\left(k^{2}, Q_{0}^{2}\right)+\left(\frac{\bar{\alpha}_{s}\left(k^{2}\right)}{N}\right) \int_{0}^{\infty} \frac{d q^{2}}{q^{2}}\left(K^{0}\left(q^{2}, k^{2}\right)\right. \\
& \left.-\alpha_{s}\left(k^{2}\right) K^{1}\left(q^{2}, k^{2}\right)\right) f\left(q^{2}\right),
\end{aligned}
$$

if attempting to find a complete solution, as proposed in [11]. Strictly speaking $\alpha_{s}\left(k^{2}\right)$ should then be the two-loop running coupling, but this will make the equation very complicated. I will just use the one-loop coupling which leads to a 2nd order differential equation in $\gamma$ space

$$
\begin{aligned}
\frac{d^{2} \tilde{f}(\gamma, N)}{d \gamma^{2}}= & \frac{d^{2} \widetilde{f}^{0}\left(\gamma, Q_{0}^{2}\right)}{d \gamma^{2}}-\frac{1}{\bar{\beta}_{0} N} \frac{d\left(\chi_{0}(\gamma) \widetilde{f}(\gamma, N)\right)}{d \gamma} \\
& -\frac{\pi}{3 \bar{\beta}_{0}^{2} N} \chi_{1}(\gamma) \widetilde{f}(\gamma, N) .
\end{aligned}
$$

This can be solved in a very similar way to the approach in Sec. IV; i.e., at leading twist it factorizes into the same form as Eq. (3.7):

$$
\mathcal{G}^{\mathrm{NLO}}\left(N, Q^{2}\right)=a^{\mathrm{NLO}}\left(Q^{2}, N\right) b^{\mathrm{NLO}}\left(Q_{0}^{2}, N\right) g_{B}\left(Q_{0}^{2}, N\right),
$$

where

$$
\begin{aligned}
a^{\mathrm{NLO}}\left(Q^{2}, N\right)= & \frac{1}{2 \pi i} \int_{1 / 2-i \infty}^{1 / 2+i \infty} \frac{1}{\gamma} \exp \left(\gamma \ln \left(Q^{2} / \Lambda^{2}\right)\right. \\
& \left.-X_{\mathrm{NLO}}(\gamma, N) /\left(\bar{\beta}_{0} N\right)\right) d \gamma .
\end{aligned}
$$

However, $X_{\mathrm{NLO}}(\gamma, N)$ is rather more complicated than the previous $X(\gamma)$. It can be expressed in the form

$$
X_{\mathrm{NLO}}(\gamma, N)=\int_{1 / 2}^{\gamma} \chi_{\mathrm{NLO}}(\hat{\gamma}, N) d \hat{\gamma}
$$

where $\chi_{\mathrm{NLO}}(\gamma, N)$ can be written as a power series in $N$ beginning at zeroth order with $\chi_{0}(\gamma)$. As seen in [11], though here ignoring any resummations in $N$, the explicit form is

$$
\begin{aligned}
\chi_{\mathrm{NLO}}(\gamma, N)= & \chi_{0}(\gamma)-N \frac{\chi_{1}(\gamma)}{\chi_{0}(\gamma)}+\frac{N^{2}}{\chi_{0}}\left(-\left(\frac{\chi_{1}(\gamma)}{\chi_{0}(\gamma)}\right)^{2}\right. \\
& \left.-\beta_{0}\left(\frac{\chi_{1}(\gamma)}{\chi_{0}(\gamma)}\right)^{\prime}\right)+\cdots,
\end{aligned}
$$

where $\chi_{2}(\gamma)$ would also appear at order $N^{2}$ if I had included it.

It is now possible to obtain some general and rather specific results using Eq. (6.16). Putting Eq. (6.20) into Eq. (6.18) we note that the leading singularities in $\gamma$ and $N$ are still both at 0 , and thus there is still no true powerlike growth. Furthermore, the singularity at $N=0$ is not affected by any of the additional terms in Eq. (6.20) beyond $\chi_{0}(\gamma)$ since in the exponent in Eq. (6.18) the $\mathcal{O}(N)$ term leads to a constant as $N \rightarrow 0$ and all higher order terms vanish in this limit. Hence, none of these terms should affect the solutions in the limit $x \rightarrow 0$, except that the $\mathcal{O}(N)$ term should affect the overall normalization, and we still expect small $x$ solutions $\sim \exp \left((\xi)^{1 / 2}\right)$ with the exponent the same as in the LO case. Hence, higher order corrections to the BFKL equation should be very subleading when calculating physical quantities. This implies that the scale for the coupling in higher order corrections should be of the same type as at LO, i.e., falling with $x$.

It is also possible to be more quantitative. Equation (6.18) can be solved using the same techniques as in Sec. IVexpanding about the saddle point leads to an ordered expansion in $\alpha_{s}\left(Q^{2}\right)$. Using Eq. (6.20) it is easy to find that the saddle point is now at

$$
\gamma^{S P N L O}\left(\bar{\alpha}_{s}\left(Q^{2}\right) / N\right)=\gamma^{0}\left(\bar{\alpha}_{s}\left(Q^{2}\right) / N\right)-\alpha_{s}\left(Q^{2}\right) \frac{\chi_{1}\left(\gamma^{0}\left[\bar{\alpha}_{s}\left(Q^{2}\right) / N\right]\right)}{\chi_{0}^{\prime}\left(\gamma^{0}\left[\bar{\alpha}_{s}\left(Q^{2}\right) / N\right]\right)}+\mathcal{O}\left(\alpha_{s}^{2}\left(Q^{2}\right)\right)
$$

Expanding as in Eq. (4.4) one finds the saddle point solution

$$
a^{\mathrm{NLO} P}\left(Q^{2}, N\right)=\frac{1}{\gamma^{S P N L O}\left(\bar{\alpha}_{s}\left(Q^{2}\right) / N, N\right)\left\{-\left[\chi_{\mathrm{NLO}}^{\prime}\left(\gamma^{S P N L O}\left[\bar{\alpha}_{s}\left(Q^{2}\right) / N\right], N\right)\right]\right\}^{1 / 2}} \exp \left(\int Q^{Q^{2}} \gamma^{S P N L O}\left(\bar{\alpha}_{s}\left(q^{2}\right) / N\right) d \ln q^{2}\right) .
$$

Further corrections can be calculated as in Sec. IV. However, this expression contains some interesting information-the dominant contribution to the running coupling corrections to the conformal part of the NLO effective splitting function. Calculating $\Gamma\left(N, Q^{2}\right)$ as a power series in $\alpha_{s}\left(Q^{2}\right)$ and transforming to $x$ space one recovers all the contributions to the splitting functions in Sec. IV. One also obtains the term $-\alpha_{s}\left(Q^{2}\right) p^{1, c o n f}\left(\bar{\alpha}_{s}\left(Q^{2}\right) \xi\right)$ which is the transformation of $-\alpha_{s}\left(Q^{2}\right) \gamma^{1}\left(\alpha_{s}\left(Q^{2}\right) / N\right)$ (with the $\beta_{0}$-dependent terms extracted), and contributions to the $\beta_{0} \alpha_{s}^{2}\left(Q^{2}\right) p\left(\bar{\alpha}_{s}\left(Q^{2}\right) \xi\right)$ splitting function. This latter term provides the scale appropriate to use in the NLO conformal splitting function using the BLM prescription at NLO [36]. This usually gives different choices for the LO and NLO scales, which could be particularly important in this case where the scale choice is so important.

Calculating $\Gamma\left(N, Q^{2}\right)$ from Eq. (6.22) the NLO conformal contribution 


$$
-\alpha_{s}\left(Q^{2}\right) \gamma^{1}\left(\alpha_{s}\left(Q^{2}\right) / N\right) \equiv-\alpha_{s}\left(Q^{2}\right) \frac{\chi_{1}\left(\gamma^{0}\left[\bar{\alpha}_{s}\left(Q^{2}\right) / N\right]\right)}{-\chi_{0}^{\prime}\left(\gamma^{0}\left[\bar{\alpha}_{s}\left(Q^{2}\right) / N\right]\right)}
$$

comes from the argument of the exponential term. The leading contribution to the $\beta_{0}$-dependent correction to this comes from the expansion of

$$
\left(-\chi_{0}^{\prime}\left(\gamma^{0}\left(\bar{\alpha}_{s}\left(Q^{2}\right) / N\right)-\alpha_{s}\left(Q^{2}\right) \frac{\chi_{1}\left(\gamma^{0}\left[\bar{\alpha}_{s}\left(Q^{2}\right) / N\right]\right)}{-\chi_{0}^{\prime}\left(\gamma^{0}\left[\bar{\alpha}_{s}\left(Q^{2}\right) / N\right]\right)}\right)\right)^{-1 / 2},
$$

to order $\alpha_{s}\left(Q^{2}\right)$ which in $\Gamma\left(N, Q^{2}\right)$ leads to the term

$$
\beta_{0} \alpha_{s}^{2}\left(Q^{2}\right) \frac{d \gamma^{0}\left(\bar{\alpha}_{s}\left(Q^{2}\right) / N\right)}{d \ln \left(\alpha_{s}\left(Q^{2}\right)\right)}\left(\frac{\left[\chi_{0}^{\prime \prime}\left(\gamma^{0}\left[\bar{\alpha}_{s}\left(Q^{2}\right) / N\right]\right)\right]^{2} \chi_{1}\left(\gamma^{0}\left[\bar{\alpha}_{s}\left(Q^{2}\right) / N\right]\right)}{\left[-\chi_{0}^{\prime}\left(\gamma^{0}\left[\bar{\alpha}_{s}\left(Q^{2}\right) / N\right]\right)\right]^{3}}\right) .
$$

It is easy to check that all other terms of $\mathcal{O}\left(\beta_{0} \alpha_{s}^{2}\left(Q^{2}\right)\right)$ are less divergent as $N \rightarrow \lambda\left(Q^{2}\right)$ than this one, including the contributions due to the $\beta_{0}$-dependent term appearing explicitly in Eq. (6.20), which are very subleading. Similarly, the contributions from the unknown $\chi_{2}(\gamma)$ will be very subleading unless $\chi_{2}(\gamma)$ is rather singular at $\gamma=\frac{1}{2}$. Taking the $\ln \left(Q^{2}\right)$ derivative of Eq. (6.23) and transforming this and Eq. (6.25) to $x$ space one may find the scale for the NLO splitting function in the same way that the scale for the LO splitting function was found in Sec. IV. However, comparing Eqs. (6.23) and (6.25) with the terms in Eq. (4.7) one notices some similarities. These are not accidental, and a careful analysis following the lines of Eqs. (4.13) to (4.20) leads to exactly the same result as at $\mathrm{LO}$ - the scale appropriate to the NLO conformal splitting function is given by

$$
\begin{aligned}
\ln \left(Q^{2} / \Lambda^{2}\right) \rightarrow & \ln \left(\widetilde{Q}^{2} / \Lambda^{2}\right)=\ln \left(Q^{2} / \Lambda^{2}\right) \\
& +\frac{(56 \zeta(3) \pi)^{1 / 2}}{4}\left(\bar{\alpha}_{s}\left(Q^{2}\right) \xi\right)^{1 / 2} .
\end{aligned}
$$

This exact equality was not at all guaranteed and is a remarkable result, implying the universality of this scale choice at all orders. It is also renormalization scheme independent, like the asymptotic form of the LO scale choice. It is undoubtedly true that the LO scale and the NLO scale will differ for finite $x$, this depending on the unknown NNLO kernel, but it shows that the asymptotic results are very simple and perturbation theory ought to be particularly convergent at small $x$. The NLO scale also matches well with the qualitative predictions obtained from consideration of the singularity structure of the full solution, as we will see below.

Using this scale at NLO we can investigate the precise effects of the NLO corrections not associated with the running coupling, the so-called conformal contributions. To begin with I simply remove the $\beta_{0}$-dependent terms from Eq. (6.13) obtaining

$$
x P\left(x, Q^{2}\right)=\alpha_{s} \exp (\lambda \xi)\left(\frac{0.068}{\left(\bar{\alpha}_{s} \xi\right)^{3 / 2}}-\alpha_{s}\left(\frac{1.23}{\left(\bar{\alpha}_{s} \xi\right)^{1 / 2}}\right)\right) .
$$

Therefore, considering $\alpha_{s}$ as a constant for the moment, we see that the NLO correction is both numerically large, and enhanced by a power of $\bar{\alpha}_{s}^{2} \xi$ compared to the LO. This latter point is really expected. Consider a leading order result of the form $\exp \left(A \bar{\alpha}_{s} \xi\right)$. When we go to NLO the coupling constant $\alpha_{s}$ becomes a renormalization scheme dependent quantity, uncertain by $\mathcal{O}\left(\alpha_{s}^{2}\right)$. In order to be consistent with the renormalization group and produce a result which is independent of renormalization scheme up to higher orders the form of the full solution must be $\exp \left(\left(A \bar{\alpha}_{s}+B \bar{\alpha}_{s}^{2}+\cdots\right) \xi\right)$, where $B$ is scheme dependent. Expanding this about the LO solution we get $\exp \left(A \bar{\alpha}_{s} \xi\right)\left(1+B \bar{\alpha}_{s}^{2} \xi+\cdots\right)$, i.e., the NLO correction is indeed a power of $\bar{\alpha}_{s}^{2} \xi$ times the LO result, exactly what we see in Eq. (6.27). From this argument it is clear that the NLO correction should be exponentiated, and we obtain

$$
x P\left(x, Q^{2}\right)=\bar{\alpha}_{s} \frac{0.068}{\left(\bar{\alpha}_{s} \xi\right)^{3 / 2}} \exp \left(\lambda \xi\left(1-6.5 \bar{\alpha}_{s}\right)\right),
$$

i.e., we obtain (slightly altered due to the removal of the $\beta_{0}$-dependent term) the publicized correction to the powerlike behavior.

However, we know that $\alpha_{s}$ is not a constant, but runs according to our scale choice at both LO and NLO. Indeed, the renormalization group argument above shows that the NLO terms in Eq. (6.12) which behave like $-0.69 \bar{\alpha}_{s} \beta_{0} \alpha_{s} \exp (\lambda \xi)$ are not of the form we would naturally expect for the NLO corrections, i.e., are not just a power of $\alpha_{s}$ higher, do not represent the order of renormalization scheme uncertainty, and are not really subleading. Resumming by absorbing them into the definition of $\alpha_{s}$ seems the only sensible thing to do. Doing this and using the scale choice (4.21) in the small $x$ limit in the expression (6.28) in both the LO and NLO parts gives

$$
\begin{aligned}
x P\left(x, Q^{2}\right) \propto & \frac{1}{\left(\alpha_{s}\left(Q^{2}\right) \xi\right)^{1 / 2}\left(\xi / \alpha_{s}\left(Q^{2}\right)\right)^{3 / 4}} \\
& \times \exp \left(1.14\left[\xi / \alpha_{s}\left(Q^{2}\right)\right]^{1 / 2}-3.0 / \alpha_{s}\left(Q^{2}\right)\right) .
\end{aligned}
$$


Therefore, it is only the LO part which gives the $x$ dependence in this limit. The NLO part gives a $Q^{2}$-dependent normalization change, which can admittedly be large [though using the $x \rightarrow 0$ limit of Eq. (4.21) tends to exaggerate the size of this at finite $x$ ], as expected from the singularity structure of the solution of the full NLO BFKL equation. Hence, using this scale choice the $\log$ of $x P\left(x, Q^{2}\right)$ is very insensitive to NLO corrections at small $x$, and we would expect the NNLO corrections to $\rightarrow 0$ as $x \rightarrow 0 .^{18}$

Therefore, I conclude that the remaining NLO corrections, after running coupling effects have been absorbed into the LO expression, are made far less significant by the effective scale used, which has been shown to be the same for LO and NLO. However, they are still potentially important at small $x$. As far as comparison with experiment is concerned the interesting question is whether these NLO corrections are significant within the current range of data available. In order to answer this question it is probably better to adopt a more sophisticated procedure, and look at the evolution not of some hypothetical gluon structure function, but of the true physical structure functions.

\section{SMALL $x$ STRUCTURE FUNCTIONS}

The previous sections have all considered the calculation of the gluon structure function obtained by integrating the solution of the BFKL equation up to the virtuality $Q^{2}$. Of course, this gluon structure function is not a real physical quantity, though it does, as we shall see, contain most of the essential information for physical quantities for asymptotically small $x$. However, we would like to see precisely how the results in the previous sections apply to real physical quantities, and how universal they are.

The generalization of the previous results to real physical scattering processes is quite straightforward. Instead of integrating the upper leg of the gluon Green's function from zero up to $Q^{2}$ we perform the convolution of the Green's function with the scattering cross section for a probe of virtuality $Q^{2}$ with a gluon of virtuality $k^{2}$ [25], i.e., Eq. (2.6) is replaced by

$$
\begin{aligned}
\mathcal{F}_{i}\left(Q^{2}, N\right)= & \int_{0}^{\infty} \frac{d k^{2}}{k^{2}} \sigma_{i, g}\left(k^{2} / Q^{2}, \alpha_{s}\left(\mu^{2}\right)\right) \\
& \times f\left(N, k^{2}, Q_{0}^{2}\right) g_{B}\left(N, Q_{0}^{2}\right) .
\end{aligned}
$$

\footnotetext{
${ }^{18}$ This result for the splitting functions as $x \rightarrow 0$ is $x P\left(x, Q^{2}\right)$ $\sim \exp \left(A_{\mathrm{LO}}\left[\xi / \alpha_{s}\left(Q^{2}\right)\right]^{1 / 2}-B_{\mathrm{NLO}} / \alpha_{s}\left(Q^{2}\right)\right)$ where $A_{\mathrm{LO}}$ is renormalization scheme independent, $B_{\mathrm{NLO}}$ is scheme dependent, and higher order corrections are claimed to be negligible. The apparent scheme dependence can be eliminated by noting that the leading order result assumed $\ln \left(\widetilde{Q}^{2} / \Lambda^{2}\right)=3.63\left(\bar{\alpha}_{s}\left(Q^{2}\right) \xi\right)^{1 / 2}$ as $x \rightarrow 0$. Including the full $\ln \left(\widetilde{Q}^{2} / \Lambda^{2}\right)=\ln \left(Q^{2} / \Lambda^{2}\right)+B_{\mathrm{LO}}+3.63\left(\bar{\alpha}_{s}\left(Q^{2}\right) \xi\right)^{1 / 2}$, where $B_{\mathrm{LO}}$ is renormalization scheme dependent, leads to $x P\left(x, Q^{2}\right)$ $\sim \exp \left(A_{\mathrm{LO}}\left[\xi / \alpha_{s}\left(Q^{2}\right)\right]^{1 / 2}+C_{\mathrm{LO}} /\left[\alpha_{s}^{2}\left(Q^{2}\right)\right]+\left(B_{\mathrm{LO}}-B_{\mathrm{NLO}}\right) / \alpha_{s}\left(Q^{2}\right)\right)$ where $C_{\mathrm{LO}}$ and $B_{\mathrm{LO}}-B_{\mathrm{NLO}}$ are scheme-independent.
}

Currently the relevant $\sigma_{i, g}\left(k^{2} / Q^{2}, \alpha_{s}\left(\mu^{2}\right)\right)$ are known at lowest nontrivial order for a number of quantities. This is order $\alpha_{s}$ for $F_{L}\left(x, Q^{2}\right)$ for both massive [37] and massless quarks [37], $F_{2}\left(x, Q^{2}\right)$ for massive quarks [25] and $\left[\partial F_{2}\left(x, Q^{2}\right) / \partial \ln Q^{2}\right]$ for massless quarks [38]. For massless quarks the lowest order result for $F_{2}\left(x, Q^{2}\right)$ is zeroth order in $\alpha_{s}$ and is infrared divergent, representing the unknown nonperturbative quark distribution function. None of the cross sections are known beyond leading order, but all diagrams accounting for the running coupling corrections at NLO for the structure functions are contained within the NLO BFKL equation.

Taking the Mellin transformation of Eq. (7.1) with respect to $\left(Q^{2} / \Lambda^{2}\right)$ leads to the simple expression

$$
\widetilde{\mathcal{F}}_{i}(\gamma, N)=\alpha_{s} h_{i, g}(\gamma) \widetilde{\mathcal{G}}(\gamma, N)
$$

where as before $\widetilde{\mathcal{G}}(\gamma, N)=\widetilde{f}\left(\gamma, N, Q_{0}^{2}\right) g_{B}\left(N, Q_{0}^{2}\right) / \gamma$, and $h_{i, g}(\gamma)$ is a function of $\gamma$ which is finite at $\gamma=0$ and $\gamma$ $=1 / 2$. Using the appropriate expression for $\widetilde{\mathcal{G}}(\gamma, N)$ the inverse Mellin transformation may be performed in the same manner as before in order to give the moment space structure functions-considering the running coupling constant BFKL equation, either LO or NLO, expanding about the same saddle points leads to an ordered solution in $\alpha_{s}$. Let us examine the simple case of $\mathcal{F}_{L}\left(N, Q^{2}\right)$ with massless quarks only. As with the gluon structure function it is impossible to actually predict this function due to the unknown $g_{B}\left(N, Q_{0}^{2}\right)$ and due to the need to regularize the BFKL equation when using the running coupling. However, the previous leading twist factorization into an incalculable $Q_{0}^{2}$-dependent function and a calculable $Q^{2}$-dependent function also applies in the same way. The function $h_{L, g}(\gamma)$ is entirely associated with the latter and does not alter the previous properties for the case of the gluon-the $Q^{2}$-dependent function is a finite unambiguous quantity with a Mellin transformation having leading singularities at $\gamma=0$ and $N=0$.

Hence, as in the case of the gluon structure function the entirely perturbative calculable quantity to consider is

$$
\Gamma_{L L}\left(Q^{2}, N\right)=\frac{\partial \ln \left(\mathcal{F}_{L}\left(N, Q^{2}\right)\right)}{\partial \ln Q^{2}} .
$$

This can be calculated for the case of the running coupling and the LO BFKL equation as in Sec. IV, with all general results being the same as in this previous case, i.e., one obtains an oscillating series in $\beta_{0} \alpha_{s}\left(Q^{2}\right)$ and a very similar apparent scale choice, as we will see below. The changes brought about by using the NLO BFKL equation with running coupling are also much the same as when considering the gluon. As stated, to get a full solution one should use the NLO BFKL equation in the form (6.15). Being instead entirely systematic one may use Eq. (6.4), and examine the results only up to NLO in $\alpha_{s}\left(\mu^{2}\right)$. Doing this one calculates the analogues of Eqs. (6.10) and (6.9). The latter is unchanged while the former is altered by the presence of $h_{L, g}(\gamma)$ into a different coefficient function $\mathcal{C}_{L}^{N L O}\left(\bar{\alpha}_{s} /\right.$ $\left.N, Q^{2} / \mu^{2}\right)$. The evaluation of this complete NLO coefficient 
function is not yet possible due to the absence of the NLO correction to $\sigma_{L, g}\left(k^{2} / Q^{2}, \alpha_{s}\left(\mu^{2}\right)\right)$. However, in order to calculate the NLO physical anomalous dimension $\Gamma_{L L}^{N L O}\left(N, Q^{2} / \mu^{2}\right)$, the analogue of Eq. (6.11), one needs only the NLO part of $\mathcal{C}_{L}^{N L O}\left(\bar{\alpha}_{s} / N, Q^{2} / \mu^{2}\right)$ containing $\ln \left(Q^{2} / \mu^{2}\right)$, which is really provided by the LO expression via the renormalization group. ${ }^{19}$ Explicitly one obtains

$$
\begin{aligned}
\Gamma_{L L}\left(N, Q^{2} / \mu^{2}\right)= & \gamma^{0}-\beta_{0} \alpha_{s}\left(\frac{\partial \gamma^{0}}{\partial \ln \left(\alpha_{s}\right)} \ln \left(Q^{2} / \mu^{2}\right)\right. \\
& \left.+\frac{\partial \gamma^{0}}{\partial \ln \left(\alpha_{s}\right)}\left(\frac{-\chi^{\prime \prime}\left(\gamma^{0}\right)}{2 \chi^{\prime}\left(\gamma^{0}\right)}-\frac{1}{\gamma^{0}}+h_{L, g}^{\prime}\left(\gamma^{0}\right)\right)\right) \\
& -\alpha_{s} \gamma^{1} .
\end{aligned}
$$

Hence, the conformal part of $\Gamma_{L L}\left(N, Q^{2} / \mu^{2}\right)$ is identical to that of $\Gamma\left(N, Q^{2} / \mu^{2}\right)$, but there is a modification to the term determining the scale. In fact, the additional term, $h_{L, g}^{\prime}\left(\gamma^{0}\right)$, is a constant at $\gamma^{0}=\frac{1}{2}$, and as such it only contributes insignificantly as $x \rightarrow 0$ : the asymptotic scale is dominated by $\left[\partial \gamma^{0} / \partial \ln \left(\alpha_{s}\right)\right]\left[-x^{\prime \prime}\left(\gamma^{0}\right) / 2 \chi^{\prime}\left(\gamma^{0}\right)\right]$ and is identical to the choice already presented for the gluon structure function. $h_{L, g}^{\prime}\left(\gamma^{0}\right)$ is important at moderate $x$, however.

Taking the transformation of Eq. (7.4) back to $x$ space and eliminating the $\beta_{0}$-dependent part (including the terms in $\gamma^{1}$ ) by setting the scale leads to a precise definition of the effective coupling constant to be used for the evolution of $F_{L}\left(x, Q^{2}\right)$ within this expansion scheme. This is presented as a function of $x$ for two choices of $Q^{2}$ in Fig. 6, and can be compared with the effective coupling for the gluon structure function (without the $\beta_{0}$-dependent terms in $\gamma^{1}$ ) in Fig. 3. Clearly in both cases the effect of the change in scale is to reduce the small $x$ coupling, and the effect becomes more important as $Q^{2}$ decreases and the size of $\hat{p}^{1}\left(\bar{\alpha}_{s}\left(Q^{2}\right) \xi\right)$ becomes larger relative to $p^{0}\left(\bar{\alpha}_{s}\left(Q^{2}\right) \xi\right)$. However, for $F_{L}\left(x, Q^{2}\right)$ the effective coupling at $x=0$ is larger than $\alpha_{s}\left(Q^{2}\right)$. This is mainly due to the $-(5 / 3) \beta_{0} \chi(\gamma)$ term in $\gamma^{1}$, but is also influenced by the first nontrivial term in the series expansion of $h_{L, g}\left(\gamma^{0}\right)$ in powers of $\left(\bar{\alpha}_{s} / N\right)$ which is negative. As $x$ decreases the effective coupling quickly decreases also, and soon falls below that in Fig. 3. This latter point is due to the $\frac{1}{2} \beta_{0}\left(\chi^{2}(\gamma)+\chi^{\prime}(\gamma)\right)$ term in $\gamma^{1}$ and the remainder of $h_{L, g}(\gamma)$ which both act to increase $\hat{p}_{L L}^{1}\left(\bar{\alpha}_{s} \xi\right)$, and hence increase the scale for the coupling. At $x=10^{-5}$ the effective coupling for $F_{L}\left(x, Q^{2}\right)$ is noticeably lower than that for the gluon, but as $x$ decreases even further the effect of the additional terms becomes less and less important, and the couplings converge.

One can now be rather quantitative about the phenomenological effects of the NLO BFKL equation and the choice of

\footnotetext{
${ }^{19}$ Equivalently one can use the formulas for the physical anomalous dimensions describing the evolution of structure functions in terms of themselves, rather than unphysical partons and coefficient functions, given in [37].
}
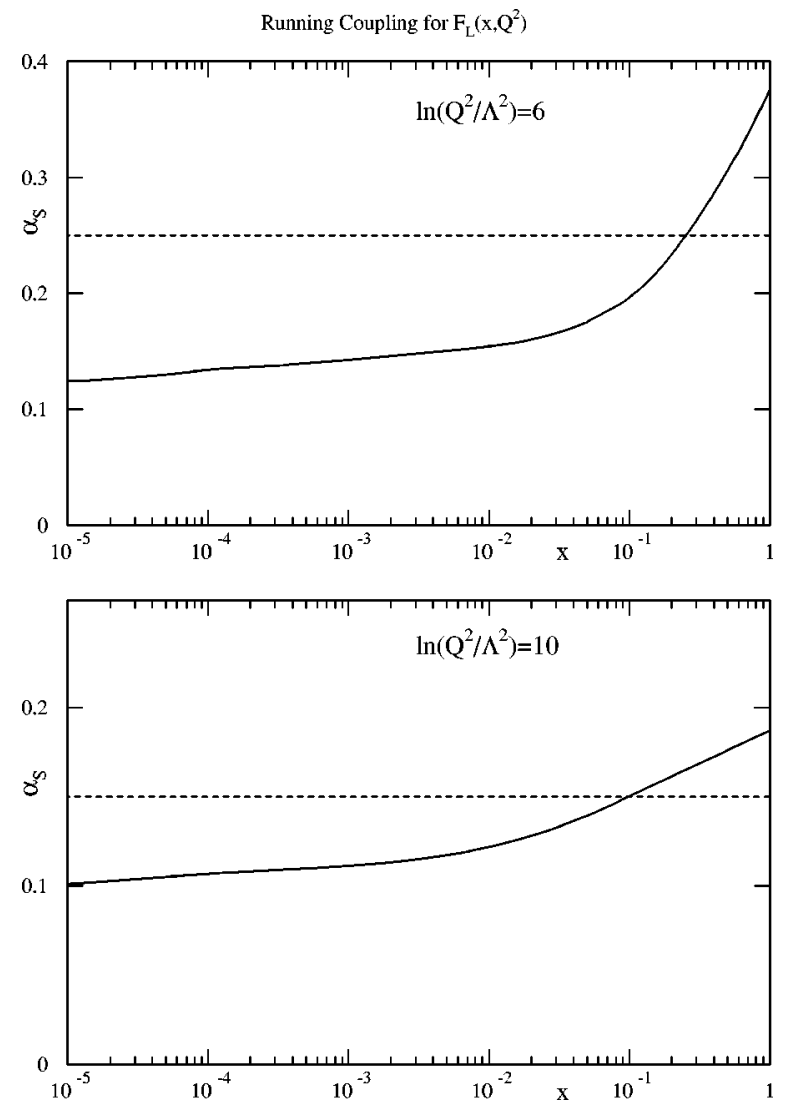

FIG. 6. The effective coupling constant for the physical splitting function $P_{L L}\left(x, Q^{2}\right)$ for $N_{F}=4$ as a function of $x$ compared to the constant values at the relevant values of $\ln \left(Q^{2} / \Lambda^{2}\right)$.

scale. Let us first make the simple scale choice $\mu^{2}=Q^{2}$. In this case we may write the physical splitting function as

$$
\begin{aligned}
\left(x / \bar{\alpha}_{s}\left(Q^{2}\right)\right) P_{L L}\left(\bar{\alpha}_{s}\left(Q^{2}\right) \xi\right)= & p_{L L}^{0}\left(\bar{\alpha}_{s}\left(Q^{2}\right) \xi\right) \\
& -\beta_{0} \alpha_{s}\left(Q^{2}\right) p_{L L}^{1, \beta}\left(\bar{\alpha}_{s}\left(Q^{2}\right) \xi\right) \\
& -\alpha_{s}\left(Q^{2}\right) p_{L L}^{1, c o n f}\left(\bar{\alpha}_{s}\left(Q^{2}\right) \xi\right) \\
\equiv & p_{L L}^{0}\left(\bar{\alpha}_{s}\left(Q^{2}\right) \xi\right) \\
& -\alpha_{s}\left(Q^{2}\right) p_{L L}^{1, \text { tot }}\left(\bar{\alpha}_{s}\left(Q^{2}\right) \xi\right),
\end{aligned}
$$

where each of the $p_{L L}^{i}\left(\bar{\alpha}_{s}\left(Q^{2}\right) \xi\right)$ may be written as a power series of the form ${ }^{20}$

$$
p_{L L}^{i}\left(\bar{\alpha}_{s}\left(Q^{2}\right) \xi\right)=\sum_{0}^{\infty} a_{n} \frac{\left(\bar{\alpha}_{s}\left(Q^{2}\right) \xi\right)^{n}}{n !} .
$$

The coefficients for the power series of the various terms in Eq. (7.5) are shown in Table I. As one can see the coefficients for all the $p_{L L}^{1}\left(\bar{\alpha}_{s}\left(Q^{2}\right) \xi\right)$ are generally much larger than those for $p_{L L}^{0}\left(\bar{\alpha}_{s}\left(Q^{2}\right) \xi\right)$.

\footnotetext{
${ }^{20}$ Actually $p_{L L}^{1, c o n f}$ has an additional term $\propto \delta(1-x) /\left(\alpha_{s}\left(Q^{2}\right)\right)$ which appears in the normal one-loop physical structure function.
} 
TABLE I. The coefficients in the power series $p_{L L}^{i}\left(\bar{\alpha}_{s}\left(Q^{2}\right) \xi\right)$ $=\Sigma_{0}^{\infty} a_{n}\left(\bar{\alpha}_{s}\left(Q^{2}\right) \xi\right)^{n} / n$ ! for the various LO and NLO contributions to the physical splitting function $P_{L L}\left(x, Q^{2}\right)$.

\begin{tabular}{rrrrr}
\hline \hline$n$ & \multicolumn{1}{c}{$p_{L L}^{0}$} & \multicolumn{1}{c}{$p_{L L}^{1, \text { tot }}$} & \multicolumn{1}{c}{$p_{L L}^{1, \beta}$} & \multicolumn{1}{c}{$p_{L L}^{1, \text { conf }}$} \\
\hline 0 & 1.00 & 0.23 & -2.00 & 1.57 \\
1 & 0.00 & 4.38 & 4.15 & 1.60 \\
2 & 0.00 & 15.87 & 11.32 & 8.29 \\
3 & 2.40 & 13.41 & -16.18 & 24.25 \\
4 & 0.00 & 86.26 & 76.03 & 35.31 \\
5 & 2.07 & 252.92 & 167.34 & 140.81 \\
6 & 17.34 & 323.08 & -81.51 & 377.69 \\
7 & 2.01 & 1699.65 & 1472.42 & 713.25 \\
8 & 39.89 & 4338.69 & 2665.07 & 2553.16 \\
9 & 168.75 & 7592.65 & 1674.16 & 6470.97 \\
10 & 69.99 & 33409.13 & 28319.16 & 14435.29 \\
11 & 661.25 & 79427.26 & 47284.56 & 47746.61 \\
12 & 1945.31 & 173361.43 & 81792.97 & 118560.14 \\
13 & 1717.68 & 657395.79 & 543255.72 & 293414.46 \\
14 & 10643.26 & 1527235.16 & 927749.64 & 905642.90 \\
15 & 25266.78 & 3833618.50 & 23539999.61 & 2256438.84 \\
\hline \hline
\end{tabular}

Using the conventional choice of scale then at leading order one would obtain the value of $\left[\partial F_{L}\left(x, Q^{2}\right) / \partial \ln Q^{2}\right]$ by convoluting the first term on the right of Eq. (7.5) with $F_{L}\left(x, Q^{2}\right)$ itself. As an appropriate choice of $F_{L}\left(x, Q^{2}\right)$ at a value of $\left(Q^{2} / \Lambda^{2}\right)=8\left(Q^{2} \sim 40 \mathrm{GeV}^{2}\right)$ I choose $F_{L}\left(x, Q^{2}\right)$ $=(x / 0.1)^{-0.3} \Theta(0.1-x)$. This is a function with the approximate shape of $F_{2}\left(x, Q^{2}\right)$ at this $Q^{2}$ and the $\Theta$ function is chosen as a crude model for the approximate $(1-x)^{6}$ fall-off at large $x$. The result for the evolution of $F_{L}\left(x, Q^{2}\right)$ is shown in the upper of Fig. 7. It increases very quickly at small $x$ due to both the shape of $F_{L}\left(x, Q^{2}\right)$ and the large splitting function at small $x$. Using the conventional scale choice one would then find the NLO evolution by using the whole of Eq. (7.5). The effect of adding in this very large negative contribution to the physical splitting function is also shown in the upper of Fig. 7. As one can see the effects are dramatic, largely killing the evolution for $x>0.0001$ and turning it sharply negative below this. ${ }^{21}$ Indeed, the NLO correction is nearly as large as the LO result for $x \sim 0.001$, and becomes dominant as $x$ decreases below this: the perturbative solution is not at all stable. Also, although we do not have measurements of $F_{L}\left(x, Q^{2}\right)$ in this range of $x$ and $Q^{2}$, similar behavior would feed through to $F_{2}\left(x, Q^{2}\right)$, and the NLO prediction is dramatically at odds with the experimental data. This is therefore a real physical example of the problems induced by the NLO BFKL equation, and is completely independent of factorization schemes and hence totally unambiguous (which is not the case for discussions of behavior of the gluon distribution in a given factorization scheme). As

\footnotetext{
${ }^{21}$ Similar behavior was found for the gluon in a particular factorization scheme $(\overline{\mathrm{MS}})$ using an incomplete calculation of the NLO anomalous dimension [39]. Using the complete anomalous dimension does not alter the qualitative results.
}
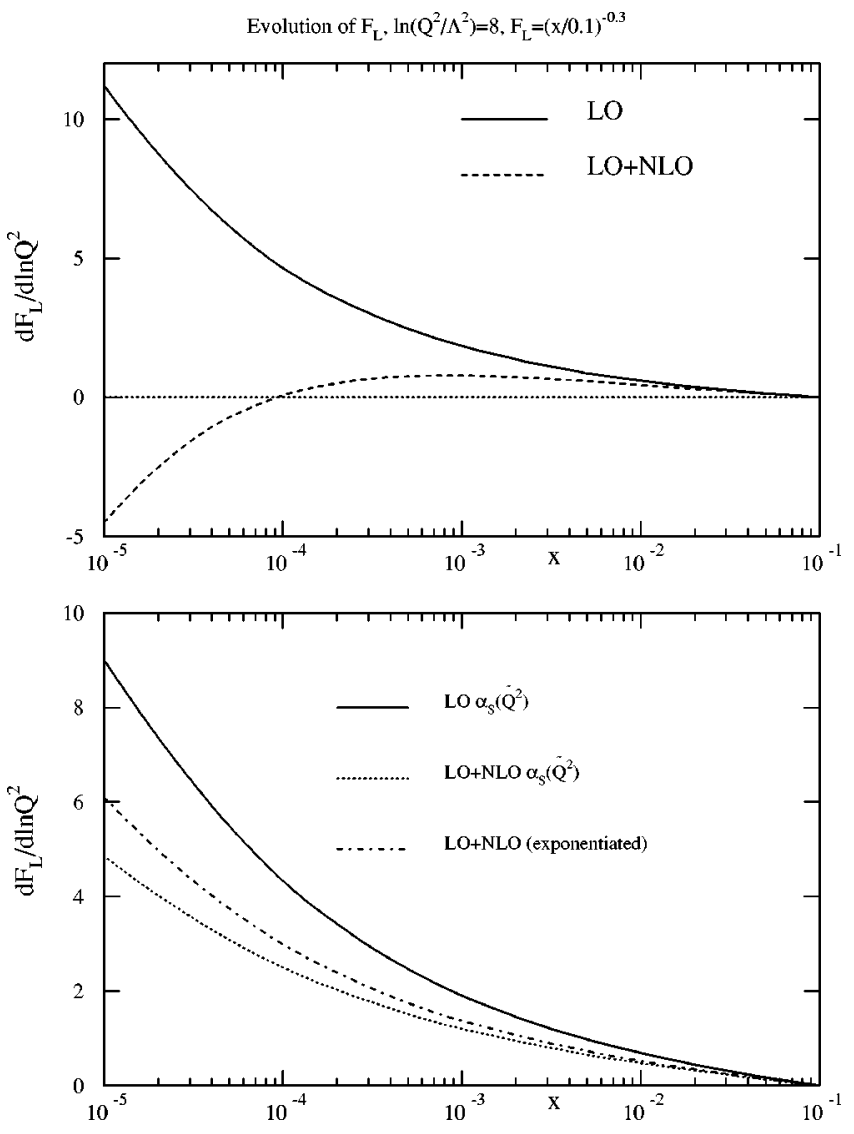

FIG. 7. The values of $\left(\partial F_{L}\left(x, Q^{2}\right) / \ln \left(Q^{2}\right)\right)$ using the resummed physical splitting functions for an input of $F_{L}\left(x, Q^{2}\right)$ $=(x / 0.1)^{-0.3} \Theta(0.1-x)$ at $\ln \left(Q^{2} / \Lambda^{2}\right)=8$ as a function of $x$. The upper figure shows the LO and LO+NLO results for the conventional scale choice $Q^{2}=\mu^{2}$. The lower figure shows the LO, $\mathrm{LO}+\mathrm{NLO}$, and $\mathrm{LO}+$ exponentiated NLO results for the $x$ dependent scale choice in this paper.

we go to lower $Q^{2}$ the coupling becomes stronger and the expected shape of the structure function becomes flatter. Both lead to the NLO corrections becoming even more important relative to the $\mathrm{LO}$, and at $Q^{2} \sim 10 \mathrm{GeV}^{2}$ the NLO correction is larger than the LO for essentially all $x$. So we see that the conventional choice for the scale leads to disastrous results.

Let us consider instead the BLM scale choice for $P_{L L}\left(x, Q^{2}\right)$. Absorbing $p_{L L}^{1, \beta}\left(\bar{\alpha}_{s}\left(Q^{2}\right) \xi\right)$ into the definition of the scale changes Eq. (7.5) to

$$
\begin{aligned}
\left(x / \bar{\alpha}_{s}\left(\widetilde{Q}^{2}\right)\right) P_{L L}\left(\bar{\alpha}_{s}\left(\widetilde{Q}^{2}\right) \xi\right)= & p_{L L}^{0}\left(\bar{\alpha}_{s}\left(\widetilde{Q}^{2}\right) \xi\right) \\
& -\alpha_{s}\left(\widetilde{Q}^{2}\right) p_{L L}^{1, c o n f}\left(\bar{\alpha}_{s}\left(\widetilde{Q}^{2}\right) \xi\right)
\end{aligned}
$$

where, as I have already noted, the LO scale is only guaranteed to be exactly the same as that to use at NLO as $x \rightarrow 0$. [Using Eq. (6.15) it is easy to show that this is true for $F_{L}\left(x, Q^{2}\right)$ in the same way as for the gluon- $h_{L, g}(\gamma)$ only introduces subleading effects as in Eq. (7.4).] The result of the evolution using the LO splitting function is shown in the 
lower of Fig. 7. It is a little smaller at the lowest values of $x$ than for $Q^{2}=\mu^{2}$, but only by $\sim 15 \%$. This is because until we get to extremely small $x$ the LO evolution is largely driven by the first term in the power series of $p_{L L}^{0}\left(\bar{\alpha}_{s}\left(\widetilde{Q}^{2}\right) \xi\right)$ due to the vanishing of the second, third and fifth terms, and relatively small fourth and sixth terms. Hence, the decrease of the coupling is only felt as a single power (and indeed there is an increase of the coupling for the highest values of $x$ ). The discrepancy between the LO results will increase at lower values of $x$. It will also increase as $Q^{2}$ gets smaller and/or as the structure function becomes less steep. It is when we include the NLO corrections that the more dramatic result is seen. The size of these now decreases for two reasons: much of the NLO correction has vanished, having been absorbed into the definition of the scale, ${ }^{22}$ and the effective coupling is now much smaller at small $x$. The result of including the NLO corrections is seen in the lower of Fig. 7. It is now a significant, but by no means overwhelming effect. As argued in the previous section renormalization scheme consistency implies that these NLO effects should really be exponentiated. The result of such an exponentiation is also shown in the lower of Fig. 7. It is clearly not dramatic, but does help the convergence of the perturbative calculation. The exponentiation will become more important as $x \rightarrow 0$. Now that I use the BLM scale choice the coupling at small $x$ is far less sensitive to $Q^{2}$ than for $\mu^{2}=Q^{2}$ and the relative importance of the NLO corrections increases far less quickly as $Q^{2}$ decreases. As shown for the case of the gluon, at asymptotically small $x$ the effective splitting function will behave like $\exp \left(1.14\left[\xi / \alpha_{s}\left(Q^{2}\right)\right]^{1 / 2}\right)$ and the exponentiated NLO corrections will lead to an $x$-independent multiplicative factor. This factor is potentially quite large, however, and the NLO effects must ultimately be treated to obtain the correct quantitative results. Nevertheless, it appears as though the LO calculation with the correct scale setting may be quite accurate in the current range of $x$ and $Q^{2}$ probed by experiment.

These results regarding $F_{L}\left(x, Q^{2}\right)$ seem very pleasing. However, phenomenologically $F_{2}\left(x, Q^{2}\right)$ is far more important since this is the quantity for which we have a great deal of data $[40,41]$. One can calculate $\left[\partial F_{2}\left(x, Q^{2}\right) / \partial \ln Q^{2}\right]$ in exactly the same way as $F_{L}\left(x, Q^{2}\right)$ simply by using the relevant $\sigma_{2, g}\left(k^{2} / Q^{2}, \alpha_{s}\left(\mu^{2}\right)\right)$ which leads to $h_{2, g}(\gamma)$ in Mellin space. Hence, in this case one obtains a direct expression for the evolution of the structure function with respect to $Q^{2}$, rather than for the structure function itself. However, inverting the Mellin transformation it is easy to see that the expression for $\left[\partial \mathcal{F}_{2}\left(N, Q^{2}\right) / \partial \ln Q^{2}\right]$ is identical to that for $\mathcal{F}_{L}\left(N, Q^{2}\right)$ up to the $h_{i, g}(\gamma)$-dependent effective coefficient function (or in this case anomalous dimension). Whereas $\mathcal{F}_{L}\left(N, Q^{2}\right)$ has a factor of $\mathcal{C}_{L}^{N L O}\left(\bar{\alpha}_{s} / N, Q^{2} / \mu^{2}\right)$ up to NLO,

\footnotetext{
${ }^{22}$ For lowish order in the power series the coefficients for $p_{L L}^{1, \beta}\left(\bar{\alpha}_{s}\left(\widetilde{Q}^{2}\right) \xi\right)$ and $p_{L L}^{1, c o n f}\left(\bar{\alpha}_{s}\left(\widetilde{Q}^{2}\right) \xi\right)$ are similar, but the former begin to dominate at higher orders, i.e., lower $x$, and become totally dominant as $n \rightarrow \infty(x \rightarrow 0)$ as demonstrated by the asymptotic results in the last section.
}

$\left[\partial \mathcal{F}_{2}\left(N, Q^{2}\right) / \partial \ln Q^{2}\right]$ has a factor of $\gamma_{2}^{N L O}\left(\bar{\alpha}_{s} / N, Q^{2} / \mu^{2}\right)$. Hence, we can write the physical evolution equation

$$
\frac{\partial \mathcal{F}_{2}\left(N, Q^{2}\right)}{\partial \ln Q^{2}}=\Gamma_{2 L}\left(Q^{2}, N\right) \mathcal{F}_{L}\left(N, Q^{2}\right),
$$

where

$$
\Gamma_{2 L}\left(N, Q^{2}\right)=\gamma_{2}^{N L O}\left(\bar{\alpha}_{s} / N, Q^{2} / \mu^{2}\right) / \mathcal{C}_{L}^{N L O}\left(\bar{\alpha}_{s} / N, Q^{2} / \mu^{2}\right) .{ }^{23}
$$

In this expression all the unknown nonperturbative physics associated with $\left[\partial \mathcal{F}_{2}\left(N, Q^{2}\right) / \partial \ln Q^{2}\right]$ and $\mathcal{F}_{L}\left(N, Q^{2}\right)$ cancel out to leave us an entirely perturbatively calculable physical anomalous dimension depending only on $Q^{2}, N$ and at finite order our choice of $\mu$.

As with $\mathcal{C}_{L}^{N L O}\left(\bar{\alpha}_{s} / N, Q^{2} / \mu^{2}\right)$ we do not know the NLO off-shell cross section and hence cannot fully calculate $\gamma_{2}^{N L O}\left(\bar{\alpha}_{s} / N, Q^{2} / \mu^{2}\right)$. Hence, we cannot calculate $\Gamma_{2 L}^{N L O}\left(N, Q^{2}\right)$ fully. However, we do know all the the effects at NLO due to the running of the coupling for both $\mathcal{C}_{L}^{N L O}\left(\bar{\alpha}_{s} / N, Q^{2} / \mu^{2}\right)$ and $\gamma_{2}^{N L O}\left(\bar{\alpha}_{s} / N, Q^{2} / \mu^{2}\right)$ and can calculate the NLO contribution to $\Gamma_{2 L}^{N L O}\left(N, Q^{2}\right)$ due to the running of the coupling and hence find the appropriate scale to use in the LO expression. This is a straightforward, though rather lengthy calculation using the NLO BFKL equation in the form (6.4), and expanding the Mellin-space solutions about the saddle point for both $\left[\partial \mathcal{F}_{2}\left(N, Q^{2}\right) / \partial \ln Q^{2}\right]$ and $\mathcal{F}_{L}\left(N, Q^{2}\right)$ in order to find the relevant parts of $\mathcal{C}_{L}^{N L O}\left(\bar{\alpha}_{s} / N, Q^{2} / \mu^{2}\right)$ and $\gamma_{2}^{N L O}\left(\bar{\alpha}_{s} / N, Q^{2} / \mu^{2}\right)$. It results in the relatively simple expression ${ }^{24}$

$$
\begin{aligned}
\Gamma_{2 L}\left(N, Q^{2} / \mu^{2}\right) \\
=\frac{h_{2, g}\left(\gamma^{0}\right)}{h_{L, g}\left(\gamma^{0}\right)}-\beta_{0} \alpha_{s} \frac{\partial \gamma^{0}}{\partial \ln \left(\alpha_{s}\right)}\left(\frac{\partial\left(h_{2, g}(\gamma) / h_{L, g}(\gamma)\right)}{\partial \gamma}\right)_{\gamma^{0}} \\
\quad \times \ln \left(Q^{2} / \mu^{2}\right)-\beta_{0} \alpha_{s} \frac{\partial \gamma^{0}}{\partial \ln \left(\alpha_{s}\right)}\left(\left(\frac{-\chi^{\prime \prime}\left(\gamma^{0}\right)}{2 \chi^{\prime}\left(\gamma^{0}\right)}-\frac{1}{\gamma^{0}}\right)\right. \\
\quad \times\left(\frac{\partial\left(h_{2, g}(\gamma) / h_{L, g}(\gamma)\right)}{\partial \gamma}\right)_{\gamma^{0}}+\frac{1}{2}\left(\frac{h_{2, g}^{\prime \prime}\left(\gamma^{0}\right)}{h_{L, g}\left(\gamma_{0}\right)}\right. \\
\left.\quad-\frac{h_{L, g}^{\prime \prime}\left(\gamma^{0}\right) h_{2, g}\left(\gamma^{0}\right)}{h_{L, g}^{2}\left(\gamma_{0}\right)}\right)+\left(\frac{\partial\left(h_{2, g}(\gamma) / h_{L, g}(\gamma)\right)}{\partial \gamma}\right)_{\gamma^{0}} \\
\left.\quad \times\left[\frac{1}{2}\left(\chi\left(\gamma^{0}\right)+\frac{\chi^{\prime}\left(\gamma^{0}\right)}{\chi\left(\gamma^{0}\right)}\right)-\frac{5}{3}\right]\right),
\end{aligned}
$$

where $h_{2, g}(\gamma)$ and $h_{L, g}(\gamma)$ can be found in [38], and $\left[h_{2, g}(\gamma) / h_{L, g}(\gamma)\right]=\Gamma_{2 L}(\gamma)=(3 / 2) \gamma+(1-\gamma)^{-1}$. As usual we can take the transformation back to $x$ space. Using the naive scale $Q^{2}=\mu^{2}$ we obtain

\footnotetext{
${ }^{23}$ Again one can use the rules for finding physical anomalous dimensions in [37].

${ }^{24}$ For reasons of simplicity I have previously defined $\Gamma_{2 L}\left(N, Q^{2} / \mu^{2}\right)$ with an additional factor of $\alpha_{s}$ [42]. This leads to no differences when calculating physical quantities.
} 
TABLE II. The coefficients in the power series $p_{2 L}^{i}\left(\bar{\alpha}_{s}\left(Q^{2}\right) \xi\right)$ $=\sum_{0}^{\infty} a_{n}\left(\bar{\alpha}_{s}\left(Q^{2}\right) \xi\right)^{n} / n$ ! for the LO and $\beta_{0}$-dependent NLO contributions to the physical splitting function $P_{2 L}\left(x, Q^{2}\right)$.

\begin{tabular}{rrr}
\hline \hline $\mathrm{n}$ & \multicolumn{1}{c}{$p_{2 L}^{0}$} & \multicolumn{1}{c}{$p_{2 L}^{1, \beta}$} \\
\hline 0 & 2.50 & -4.00 \\
1 & 1.00 & 9.39 \\
2 & 1.00 & 36.60 \\
3 & 7.01 & 6.27 \\
4 & 5.81 & 239.73 \\
5 & 13.40 & 687.03 \\
6 & 58.11 & 771.35 \\
7 & 64.74 & 5281.50 \\
8 & 196.83 & 13213.51 \\
9 & 649.89 & 24043.80 \\
10 & 930.65 & 111578.92 \\
11 & 3034.70 & 265509.09 \\
12 & 8527.87 & 613964.05 \\
13 & 15046.02 & 2311855.03 \\
14 & 48434.53 & 5521425.31 \\
15 & 124600.51 & 14458201.96 \\
\hline \hline
\end{tabular}

$$
\begin{aligned}
\left(x / \bar{\alpha}_{s}\left(Q^{2}\right)\right) P_{2 L}\left(\bar{\alpha}_{s}\left(Q^{2}\right), x\right)= & \delta(1-x) / \bar{\alpha}_{s}\left(Q^{2}\right) \\
& +p_{2 L}^{0}\left(\bar{\alpha}_{s}\left(Q^{2}\right) \xi\right) \\
& -\beta_{0} \alpha_{s}\left(Q^{2}\right) p_{2 L}^{1, \beta}\left(\bar{\alpha}_{s}\left(Q^{2}\right) \xi\right),
\end{aligned}
$$

where the $p_{2 L}^{i}\left(\bar{\alpha}_{s}\left(Q^{2}\right) \xi\right)$ are power series of the form (7.6), and the LO physical splitting function has a zeroth order term proportional to a $\delta$ function. As is now standard we can find the correct scale by eliminating all $\beta_{0}$-dependent NLO terms. This is a little more involved than the previous cases, but in the asymptotic limit reduces to exactly the same result. As $\gamma^{0} \rightarrow \frac{1}{2}$ if we keep only the most divergent part in the third term on the right in Eq. (7.9) then we have the condition that the $x$-space version of

$$
\begin{aligned}
& \frac{\partial \gamma^{0}}{\partial \ln \left(\alpha_{s}\right)}\left(\frac{\partial\left(h_{2, g}(\gamma) / h_{L, g}(\gamma)\right)}{\partial \gamma}\right)_{\gamma^{0}} \ln \left(Q^{2} / \widetilde{Q}^{2}\right) \\
& \quad+\frac{\partial \gamma^{0}}{\partial \ln \left(\alpha_{s}\right)}\left(\frac{-\chi^{\prime \prime}\left(\gamma^{0}\right)}{2 \chi^{\prime}\left(\gamma^{0}\right)}\right)\left(\frac{\partial\left(h_{2, g}(\gamma) / h_{L, g}(\gamma)\right)}{\partial \gamma}\right)_{\gamma^{0}},
\end{aligned}
$$

must vanish. Since in this limit $\left[\left(\partial\left[h_{2, g}(\gamma) / h_{L, g}(\gamma)\right]\right) /\right.$ $\partial \gamma]_{\gamma^{0}} \rightarrow 5 / 2$ this is precisely the same condition as we found for the gluon and for $\mathcal{F}_{L}\left(N, Q^{2}\right)$, and we obtain exactly the same asymptotic scale (4.21). Indeed, if we attach any physical process to the top of the gluon ladder we will always obtain solutions for physical quantities in the same manner: the physical anomalous dimension or coefficient function will be determined from the part of the solution which has factorized, is $Q^{2}$ dependent, and is influenced by the diffusion into the ultraviolet. Hence, we would always expect physical quantities to be controlled by the same asymptotic scale.

Being more precise we may find the $x$-space version of Eq. (7.9) as a power series in $\bar{\alpha}_{s}\left(Q^{2}\right) \xi$. The coefficient functions for the known $p_{2 L}^{i}\left(\bar{\alpha}_{s} \xi\right)$ are shown in Table II. Using these series we can solve exactly for the scale down to some finite value of $x$. The effective coupling to be used when calculating the small $x$ evolution of $F_{2}\left(x, Q^{2}\right)$ in terms of $F_{L}\left(x, Q^{2}\right)$ is actually very similar to that for the evolution of $F_{L}\left(x, Q^{2}\right)$ over the whole range of $x$. They become identical as $x \rightarrow 0$, but are only slightly different even as $x \rightarrow 1$.

If we examine the value of $\left[\partial F_{2}\left(x, Q^{2}\right) / \partial \ln Q^{2}\right]$ for the given input for $F_{L}\left(x, Q^{2}\right)$ using the LO physical anomalous dimension we find that the decrease in going from the choice $Q^{2}=\mu^{2}$ to the effective scale is a little larger than when examining $\left[\partial F_{L}\left(x, Q^{2}\right) / \partial \ln Q^{2}\right]$. This is simply because the terms in the power series for $p_{2 L}^{0}\left(\bar{\alpha}_{s} \xi\right)$ are not as small as those for $p_{L L}^{0}\left(\bar{\alpha}_{s} \xi\right)$, and so higher terms in the series, where powers of the coupling are used, are proportionally more important. Since we do not actually know the value of $p_{2 L}^{1, \text { conf }}\left(\bar{\alpha}_{s} \xi\right)$ it is impossible to evaluate the NLO effects, with or without the scale setting, but I imagine they are of similar importance to the those for $F_{L}\left(x, Q^{2}\right)$. They will certainly lead to the same general result, i.e., the LO expression $\sim \exp \left(1.14(\xi) / \alpha_{s}\left(Q^{2}\right)\right)^{1 / 2}$ as $x \rightarrow 0$ with the exponentiated NLO corrections leading to an $x$-independent multiplicative factor.

I note that within this picture there is no way of predicting inputs for structure functions (or partons) at some fixed $Q_{I}^{2}$. However, since the evolution generates no true powerlike behavior there may well be no growth at $x \rightarrow 0$ stronger than the soft Pomeron. I see no reason to believe the values for the intercepts calculated by putting some infrared cutoff on the BFKL equation for running coupling, which are both cutoff method and scale dependent. However, at the sort of values of $x$ we consider in practice, $x=10^{-2} \rightarrow 10^{-5}$, the perturbative evolution can generate a rise at small $x$ which appears to be like an effective power over this restricted range in $x$. In broad terms this will not be dissimilar to that generated by the NLO in $\alpha_{s}\left(Q^{2}\right)$ evolution, but will be different in detail. Perhaps the best method for attempting to predict the shape of a structure function at a given input scale is to demand that the general form of the structure functions are as insensitive to changes in starting scale as possible [42]. In this way the inputs are determined largely by the form of the evolution, and hence the effective physical splitting functions. Since the small $x$ evolutions of $F_{2}\left(x, Q^{2}\right)$ and $F_{L}\left(x, Q^{2}\right)$ are related in a calculable manner this imposes a precise consistency requirement on the small $x$ inputs of the two. A more detailed study of study of this would be interesting, though an obvious conclusion is that the shape of $F_{2}\left(x, Q^{2}\right)$ and $F_{L}\left(x, Q^{2}\right)$ with $x$ should be roughly the same at all $Q^{2}$ and hence at $Q_{I}^{2}$ (see below).

\section{PHENOMENOLOGICAL CONSEQUENCES}

Armed with the small $x$ scale choices for the physical structure functions, it is now possible to do a phenomeno- 
logical analysis. The inclusion of the input singlet quark distribution, or equivalently the inclusion of $\Gamma_{L 2}\left(N, Q^{2}\right)$ and $\Gamma_{22}\left(N, Q^{2}\right)$ is easy since at LO these are related in a simple manner to $\Gamma_{L L}\left(N, Q^{2}\right)$ and $\Gamma_{2 L}\left(N, Q^{2}\right)$, respectively [37]. Furthermore, they have only a small effect. Much more important is the treatment of the LO terms in the physical splitting functions which are less singular than $1 / x$ as $x \rightarrow 0$. As shown in [42] a full LO analysis should include all such terms at lowest order in $\alpha_{s}$ as well as all terms in the LO small $x$ expansions considered so far in this paper. A correct extension of [42], which used the simple scale choice $Q^{2}$ $=\mu^{2}$, would involve the full LO, in $\alpha_{s}$ as well as $\ln (1 / x)$, physical splitting functions with the scale choice determined not only by the NLO running coupling effects considered in this paper, but also by the $x$-finite NLO in $\alpha_{s}$ running coupling effects.

Consideration of the NLO in $\alpha_{s}$ running coupling effects leads to additional important scale changes away from $Q^{2}$ $=\mu^{2}$ at high $x$. The evolution of the nonsinglet structure function $F_{2}^{N S}\left(x, Q^{2}\right)$ was considered in [43] where it was found that the appropriate scale to use is

$$
\widetilde{Q}^{2}=Q^{2} \frac{(1-x)}{x^{2}} k(x),
$$

where $k(x)$ is a relatively smooth function of $x$ from $0 \rightarrow 1$, $k(x) \approx 0.15$. Careful consideration shows that such a scale change (with some regularization as $x \rightarrow 1$ ) must be implemented at high $x$ for quark driven processes, leading to a larger coupling and quicker evolution. There are also nontrivial high $x$ effects in the gluon driven processes due to the NLO in $\alpha_{s}$ running coupling terms. This changes the detailed form of the effective coupling already presented in Fig. 6 for values of $x$ above approximately $x=0.05$. For values of $x$ below this the finite $x$ effects on the scale fall away quickly.

One particular consequence of including the full $\mathcal{O}\left(\alpha_{s}\right)$ effective splitting functions is that like $P_{g g}\left(z, Q^{2}\right)$, $P_{L L}\left(z, Q^{2}\right)$ actually leads to a fall with $Q^{2}$ for high values of $z$, the rise only setting in when the small $x$ terms become dominant. Hence, the fact that the effective coupling for $P_{L L}\left(z, Q^{2}\right)$ is actually large at high $z$ increases this negative contribution, whereas the smaller coupling at small $z$ decreases the positive contribution, as we already know. This means that, looking at the complete convolution leading to the evolution of $F_{L}\left(x, Q^{2}\right)$, the increased negative contribution at high $z$ leads to the full scale-fixed $\mathrm{LO}$ evolution being reduced compared to the full $Q^{2}=\mu^{2}$ LO evolution more than the consideration of small $x$ effects only in Fig. 7 suggests. Inclusion of the high $x$ terms at NLO has precisely the opposite effect: this time the positive contribution to the evolution from high $z$ due to the $\mathcal{O}\left(\alpha_{s}^{2}\right)$ terms is enhanced, as well as the known effect of the negative contribution from small $z$ being much reduced in size. Hence, the negative NLO correction at small $x$ is significantly reduced compared to that seen in Fig. 7. Details will be shown in a future paper [44], but the apparent convergence of the perturbative expansion is considerably better even than that implied in the previous section.
TABLE III. Comparison of quality of fits using full leading order [including $\ln (1 / x)$ terms] renormalization scheme consistent expression, with BLM scale setting and the NLO in $\alpha_{s}\left(Q^{2}\right)$ fit [47]. The references to the data can be found in [47].

\begin{tabular}{lrrr}
\hline \hline & & \multicolumn{2}{c}{$\chi^{2}$} \\
\cline { 3 - 4 } Experiment & Data & LO $(x)$ & MRST \\
\hline H1 $F_{2}^{e p}$ & 221 & 149 & 164 \\
ZEUS $F_{2}^{e p}$ & 204 & 246 & 270 \\
BCDMS $F_{2}^{u p}$ & 174 & 241 & 249 \\
NMC $F_{2}^{\mu p}$ & 130 & 118 & 141 \\
NMC $F_{2}^{\mu d}$ & 130 & 81 & 101 \\
NMC $F_{2}^{\mu n} / F_{2}^{\mu p}$ & 163 & 176 & 187 \\
SLAC $F_{2}^{\mu p}$ & 70 & 87 & 119 \\
E665 $F_{2}^{\mu p}$ & 53 & 59 & 58 \\
E665 $F_{2}^{\mu d}$ & 53 & 61 & 61 \\
CCFR $F_{2}^{\nu N}$ & 66 & 57 & 93 \\
CCFR $F_{3}^{\nu N}$ & 66 & 65 & 68 \\
Total & 1330 & 1339 & 1511 \\
\hline \hline
\end{tabular}

I leave a full discussion of the implementation of a full LO in $\alpha_{s}$ and $\ln (1 / x)$ (denoted by LORSC) global fit using scale setting in physical anomalous dimensions to a future paper. ${ }^{25}$ Details of such a (slightly approximate) fit have already been briefly reported in [27], and here I report the most important consequences.

(1) Compared to the most recent NLO in $\alpha_{s}\left(Q^{2}\right)$ global fit [47] the quality of the $\chi^{2}$ is improved from 1511 to 1339 for 1330 structure function data points. (Constraints from nonstructure function data, e.g., prompt-photon, Drell-Yan, etc. at high $x$ are imposed in the same manner for both.) A breakdown of the $\chi^{2}$ for each experiment is shown in Table III. This extremely statistically significant improvement is achieved in all regions of $x$ and $Q^{2}$-the scale choice (8.1) helping at high $x$ and the resummation of $\bar{\alpha}_{s} \xi$ terms coupled with the scale choice helping at small $x$. The value of the LO coupling is set at $\alpha_{s}\left(M_{Z}^{2}\right)=0.116$, where this LO value is unambiguous, contrary to the normal case at $\mathrm{LO}$, because the scale choice has been determined unambiguously. The effects of varying the coupling remain to be investigated. A standard NLO in $\alpha_{s}$ fit with BLM inspired scale fixing has also recently been performed [48] with less impressive results, particularly at small $x$.

(2) Since the procedure for calculating the evolution is very different from the NLO in $\alpha_{s}\left(Q^{2}\right)$ approach, predictions resulting from the best fit are significantly altered. For example, the additional terms in powers of $\bar{\alpha}_{s} \xi$ in $p_{2 L}^{0}\left(\bar{\alpha}_{s} \xi\right)$ compared to the NLO in $\alpha_{s}\left(Q^{2}\right)$ approach more than compensates for the decrease in the effective coupling at moderate $x$ and $Q^{2}$, leading to a smaller $F_{L}\left(x, Q^{2}\right)$ (very similar to

\footnotetext{
${ }^{25}$ It is also necessary to treat the heavy partons in a consistent manner. The way to do this in the context of the full LO physical anomalous dimensions with $Q^{2}=\mu^{2}$ was presented briefly in [45], and will be presented in more detail in a future paper [46].
} 
that predicted in [42] if $Q^{2} \geqslant 15 \mathrm{GeV}^{2}$ ) being required to obtain a similar rate of evolution for $F_{2}\left(x, Q^{2}\right)$. Predictions for other processes, e.g., Drell-Yan production, are potentially very different in the two approaches.

(3) There is a failure of the NLO in $\alpha_{s}\left(Q^{2}\right)$ approach at small $x$ for $Q^{2} \leqslant 2-3 \mathrm{GeV}^{2}$. This can be seen in two ways. If the gluon [and hence $\left.F_{L}\left(x, Q^{2}\right)\right]$ is required to be positive definite down to $Q^{2}<1 \mathrm{GeV}^{2}$ then the value of $\left[\partial F_{2}\left(x, Q^{2}\right) / \partial \ln Q^{2}\right]$ becomes too large for $Q^{2} \leqslant 2-3 \mathrm{GeV}^{2}$ [49] (a plot can be found in [50]), as can be seen by comparing the data with the prediction from a GRV type parameterization [51]. Alternatively, the value of $\left[\partial F_{2}\left(x, Q^{2}\right) / \partial \ln Q^{2}\right]$ can be made correct down to $\sim 1 \mathrm{GeV}^{2}$, at the expense of having a valencelike gluon distribution, and hence odd shaped $F_{L}\left(x, Q^{2}\right)$ (see below), at $Q^{2}=1 \mathrm{GeV}^{2}$, and hence negative gluon and $F_{L}\left(x, Q^{2}\right)$ below this [47,50]. Each case demonstrates that the NLO in $\alpha_{s}\left(Q^{2}\right)$ approach is breaking down at $Q^{2} \sim 2-3 \mathrm{GeV}^{2}$ at small $x{ }^{26}$ While this might not seem surprising since there are many potential reasons for this failure [higher twist, higher orders and of course $\ln (1 / x)$ resummations], it is a problem not shared by the full LORSC fit with the correct scale (even though it is a considerably better fit at small $x$ than in [47]). Because the small $x$ effective coupling becomes proportionally smaller compared to $\alpha_{s}\left(Q^{2}\right)$ as we tend to lower $Q^{2}$, and because, as seen in Table I, the coefficients in the expansion of $p_{L L}^{0}\left(\bar{\alpha}_{s} \xi\right)$ are small, the evolution of $F_{L}\left(x, Q^{2}\right)$ is slowed down at very small $x$ and $Q^{2}$ compared to the NLO in $\alpha_{s}\left(Q^{2}\right)$ approach. Hence, the $F_{L}\left(x, Q^{2}\right)$ predicted by the global fit does not evolve backwards into a pathological form at $Q^{2}=1 \mathrm{GeV}^{2}$. This is shown in Fig. 8 where I compare the predicted $F_{L}\left(x, Q^{2}\right)$ with that obtained from the MRST analysis at $Q^{2}=1.2 \mathrm{GeV}^{2}$. Clearly the shape of the LORSC $F_{L}\left(x, Q^{2}\right)$ is not dissimilar to that of $F_{2}\left(x, Q^{2}\right)$ at the same $Q^{2}$, while the MRST $F_{L}\left(x, Q^{2}\right)$ is rather odd, though it looks sensible by about $2 \mathrm{GeV}^{2}$. (The rise at very small $x$ in the MRST curve is due to the small quark contribution becoming dominant over the large but valencelike gluon contribution.) Evolving downwards the MRST $F_{L}\left(x, Q^{2}\right)$ dips down to negative values at about $1 \mathrm{GeV}^{2}$ while the LORSC $F_{L}\left(x, Q^{2}\right)$ will clearly be sensible to much lower values (this will be investigated in detail in [44]). Since the effective coupling at small $x$ is so small it seems reasonable to believe that the full LORSC calculation should really represent the physics down to low $Q^{2}$, as it does, whereas even if the NLO in $\alpha_{s}\left(Q^{2}\right)$ approach

\footnotetext{
${ }^{26}$ I note that despite reports to the contrary an analysis of data using the leading $\ln (1 / x)$ terms with $\alpha_{s}\left(Q^{2}\right)$ does not fail in any more dramatic a manner than this. As shown in [45], using the LO physical anomalous dimensions to perform the analysis, rather than some factorization scheme which leads to extremely ambiguous results at small $x$, a fit of even better quality than the NLO in $\alpha_{s}\left(Q^{2}\right)$ fit can be achieved. The only failings are that the pathological behavior in the predicted $F_{L}\left(x, Q^{2}\right)$ sets in at very slightly higher $Q^{2}$, and of course the NLO corrections using this approach appear to be huge.
}

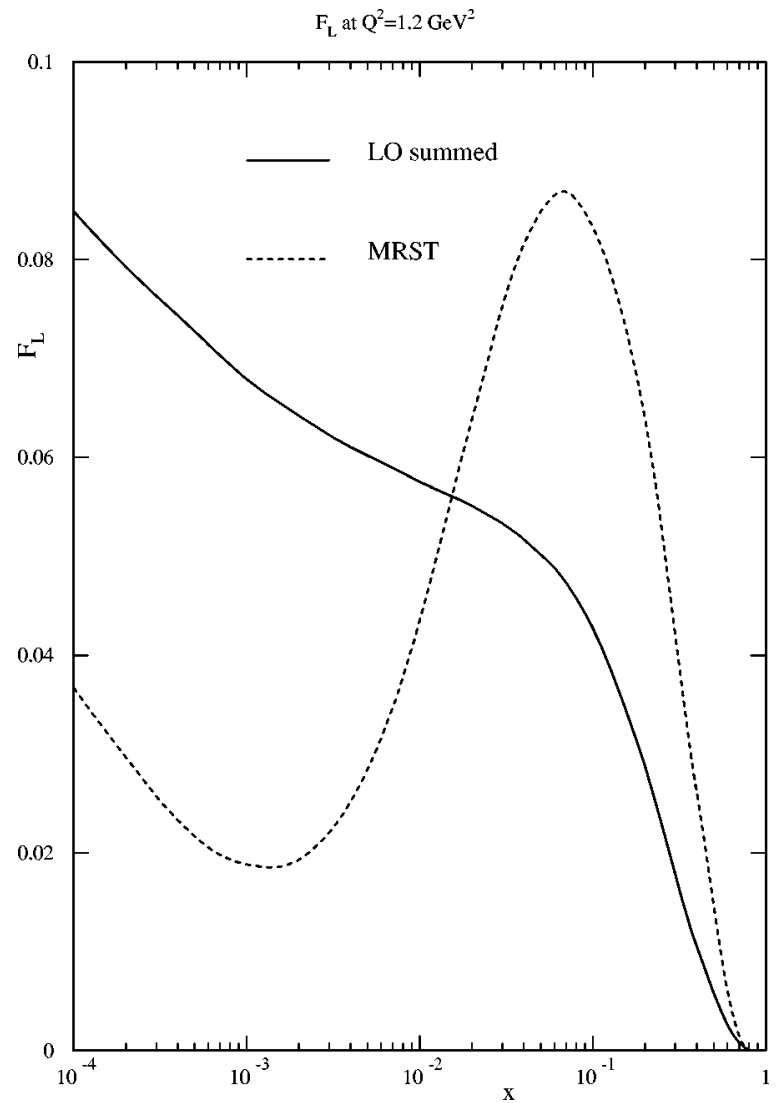

FIG. 8. Comparison of the predictions for $F_{L}\left(x, Q^{2}\right)$ at $Q^{2}$ $=1.2 \mathrm{GeV}^{2}$ from the global fit performed in this paper and the NLO in $\alpha_{s}\left(Q^{2}\right)$ fit in [45].

had worked we would not have known why. ${ }^{27}$

Hence, all details of the phenomenology of the scale fixed LORSC analysis seem very satisfactory, being a distinct improvement on the standard approach and the LORSC analysis with $Q^{2}=\mu^{2}$. As a word of caution, the analysis presented is still a little approximate, and all quantitative results are likewise approximate. A more careful detailed analysis will appear soon, though it would be very surprising if the same quality fit were not achieved simply by a slight alteration of input parameters and hence very slightly different predictions.

\section{CONCLUSIONS}

I have presented a full discussion of the effect of the NLO corrections to the BFKL equation. I have shown that if one resums the $\ln \left(k^{2} / \mu^{2}\right)$ terms into a running coupling constant, as must be roughly correct, this alters the whole structure of the solution to the BFKL equation. As previously pointed out $[15,21]$, at leading twist it leads to the solution factorizing into a input dependent part which requires regularization, i.e., is infrared renormalon contaminated, and a $k^{2}$-dependent

\footnotetext{
${ }^{27}$ A recent discussion of the "Caldwell plot'" using the LO BFKL equation with running coupling, though with very different techniques from those used in this paper, appears in [52].
} 
part which is well defined. The degree of uncertainty associated with the input part is shown to have exactly the behavior predicted by Mueller [23,7]. However, this ambiguity affects the input part only, not the whole solution. I note that the evolution part as a function of $\gamma$ and $N$ no longer has singularities to the right of zero for either $\gamma$ or $N$, a result which has previously been noted $[15,20]$, but seems to have been universally ignored. Hence, this calculable $k^{2}$-dependent solution has no true powerlike behavior in either $k^{2}$ or $x$ - the hard Pomeron intercept is zero. These results require no assumptions at all. If one takes the running of the coupling in the BFKL equation seriously, the input term is indeterminate unless $13 \beta_{0}^{2} \alpha_{s}^{3}\left(Q_{0}^{2}\right) \xi \ll 1$, and the evolution term is well defined and calculable, and has no true powerlike behavior. This is not difficult to understand in a qualitative manner. It has long been known that the typical virtuality of a gluon in the ladder representing the BFKL Green's function has a mean of order $k^{2}$, but a deviation of order $\left(\bar{\alpha}_{s} \xi\right)^{1 / 2}$ [22]. I have shown that the diffusion of $k^{2}$ into the infrared influences only the input dependent solution, the strong coupling then leading to infrared renormalons, while the $k^{2}$-dependent part is influenced only by the ultraviolet diffusion. This means that as one goes to smaller and smaller $x$ the appropriate scale becomes larger and larger, the coupling weaker and weaker (like $\xi^{-1 / 2}$ ), and the growth from the $\ln (1 / x)$ terms is sufficiently weakened by the coupling to destroy the powerlike behavior.

Using the LO BFKL solution with running coupling I have argued that in order to investigate perturbatively calculable physics one must investigate physical anomalous dimensions [37], or splitting functions, which tell one how unambiguous physical quantities evolve in terms of each other, and hence are themselves unambiguous, i.e., independent of factorization schemes or scales. This is important when using a small $x$ expansion even at low orders due to large factorization scheme uncertainties, but is now vital in order to obtain well-defined, perturbatively calculable results. While, of course, it is ultimately necessary to use real structure functions $F_{2}\left(x, Q^{2}\right)$ and $F_{L}\left(x, Q^{2}\right)$, one may for simplicity work with an unphysical, but unambiguously defined gluon structure function $G\left(x, Q^{2}\right)$. By calculating the solutions for the $Q^{2}$-dependent factors of the structure functions about the saddle points, one obtains ordered power series in $\beta_{0} \alpha_{s}\left(Q^{2}\right)$ for the physical anomalous dimensions. While these series appear to be very badly convergent, the coefficients oscillate in sign, rendering them summable. I hypothesize that one can approximate the whole result by using the BLM scale fixing procedure [24] absorbing the NLO $\beta_{0}$-dependent term into the definition of the scale used in the LO expression. This results in an effective coupling of the form $1 /\left(\beta_{0}\left[\ln \left(Q^{2} / \Lambda^{2}\right)+3.63\left[\bar{\alpha}_{s}\left(Q^{2}\right) \xi\right]^{1 / 2}\right]\right)$ as $x \rightarrow 0$. For different physical variables the moderate $x$ couplings are slightly different but the asymptotic form is universal. It is not guaranteed that this choice of coupling is really correct. However, the explicit NNLO calculation supports the procedure strongly, and it is also consistent with the qualitative features one knows must be associated with the full summation, i.e., it smooths out the powerlike growth in $x$ in pre- cisely the correct manner, as well as the picture of ultraviolet diffusion.

Examining the full NLO BFKL equation I find that as far as running coupling is concerned by far the dominant effect is produced solely by the $\ln \left(k^{2} / \mu^{2}\right)$ term. All additional NLO $\beta_{0}$-dependent corrections lead to modifications to the physical splitting functions which are not only numerically small, but are reduced by a factor of $\left(\bar{\alpha}_{s}\left(Q^{2}\right) \xi\right)^{-1 / 2}$. This indicates that it is likely that at all orders the $\ln \left(k^{2} / \mu^{2}\right)$ terms will lead to the dominant small $x$ effects due to running of the coupling. Indeed, at NLO the contribution to the physical splitting function from this term is also dominant to the conformal corrections by $\left(\bar{\alpha}_{s}\left(Q^{2}\right) \xi\right)^{1 / 2}$. The latter are of the form expected from a renormalization group argument, i.e., a factor of $\alpha_{s}\left(\bar{\alpha}_{s} \xi\right)$ up on the $\mathrm{LO}$ expression, while the running coupling effect is of an unexpected, more leading form, and essentially demands to be resummed. I also proved that if one assumes the dominance of the $\ln \left(k^{2} / \mu^{2}\right)$ terms the appropriate scale to use at NLO is precisely the same as the LO scale as $x \rightarrow 0-a$ result which was by no means guaranteed to be true and seems strongly suggestive of the correctness of the approach. It also implies that perturbation theory at small $x$ should be particularly convergent. Using this effective scale choice in the coupling I find that the remaining, conformal NLO corrections to the physical anomalous dimensions are much more under control than for the scale choice $Q^{2}=\mu^{2}$ due to the smallness of the effective coupling as $x$ $\rightarrow 0$. At all $x$ and $Q^{2}$ they are subdominant to the LO result, although they can be significant, and in the region of $x$ and $Q^{2}$ probed at the DESY ep collider HERA they are numerically quite small.

An analysis of data using the full LO physical splitting functions containing both leading in $\ln (1 / x)$ terms and all $\mathcal{O}\left(\alpha_{s}\right)$ terms, with scale fixing appropriate to this combined expansion scheme, is very successful. It produces a far better fit to data than conventional approaches, and also predicts an $F_{L}\left(x, Q^{2}\right)$ of the same shape as $F_{2}\left(x, Q^{2}\right)$ down to $Q^{2}$ $=1 \mathrm{GeV}^{2}$, and possibly below. In fact, it seems to work perfectly over the whole range of parameter space one might hope. The fit to $F_{2}\left(x, Q^{2}\right)$ also leads to predictions for other quantities such as $F_{L}\left(x, Q^{2}\right)$ (difficult to measure), $F_{2}^{c}\left(x, Q^{2}\right)$ (not much different from the standard approach) and DrellYan production (if the necessary BFKL coefficient functions were calculated).

Since the coupling at small $x$ is weak, seemingly at all orders, one may be optimistic that it is possible to use even LO perturbation theory down to very low $Q^{2}$ at small $x$. Indeed, the prediction is that the corrections at NNLO and beyond will be insignificant due to the fall of the coupling overwhelming all possible enhancement due to small $x$ terms. However, there are still potentially important higher twist $\left(\Lambda^{2} / Q^{2}\right)$ contributions. Nevertheless, the weakness of the coupling may make one hope that the small $x$ higher twist effects are strongly suppressed, for example a weaker coupling would certainly delay the onset of such effects as shadowing [12] rather significantly. Also, I note that within the small $x$ expansion there are no infrared renormalons in the calculation of the physical anomalous dimensions. Since renormalons lead to ambiguities which must be cancelled by 
higher twist ambiguities they are normally taken to be estimates of the size of these higher twist contributions-indeed, the scale fixing for nonsinglet evolution at high $x$ [43] does imply renormalons of the type already calculated [53]. The absence of the renormalons at small $x$ makes the author at least optimistic about the smallness of higher twist effects. Some small $x$ higher twist calculations have already been performed [54]. However, since the full physical picture at leading twist only appears when performing a full resummed $\ln (1 / x)$ calculation including running coupling effects, a true picture of the higher twist contributions may sadly require similar sophistication (if this is possible). I certainly feel that any renormalon calculations performed at fixed order in $\alpha_{s}$ may not be representative of the true small $x$ higher twist contributions. If the full $\mathrm{LO}$, with resummed terms and scale fixing, analysis is indeed successful to very low $Q^{2}$ I would regard this as empirical evidence, if by no means a proof, of the smallness of higher twist corrections at small $x$.

I have commented on other approaches to the NLO BFKL equation throughout this paper. There have also recently been alternative attempts to improve the apparent bad convergence of the perturbative series which are somewhat orthogonal to the line taken in this paper. In [10] and [11] progress is made by finding resummations which improve the convergence of the expansion of the kernel, thus implying a sensible, stable pomeron intercept. I have no argument with this approach and believe that for single scale processes it is vital for obtaining a stable expansion for general values of $x$. However, I also believe that for structure functions it leads to effects that are completely subdominant to those induced by the running of the coupling. If my assumption about the running coupling in the kernel being accounted for by the effective $x$-dependent coupling in physical quantities has any truth in it, it makes resummations of the conformal part of the kernel unimportant since the higher orders are so greatly weakened by the reduction in the coupling. Hence, while the work in $[10,11]$ is certainly interesting, I believe it may be unimportant for the real physical results, at least as far as structure functions are concerned.

Also, there has very recently been a proposal to adopt the BLM scale fixing procedure at the level of the eigenvalues of the kernel [55]. This is similar, though not identical to the proposal for the change in coupling proposed in [56] when the $N_{f}$-dependent corrections to the NLO kernel were known. It avoids all the running coupling effects I consider in Sec. IV, picking up only those in $\gamma^{1}$ in Sec. VI, i.e., the $\frac{1}{2} \beta_{0}\left(\chi^{2}(\gamma)+\chi^{\prime}(\gamma)\right)$ and $-(5 / 3) \beta_{0} \chi(\gamma)$ terms. This leads to a scale change $\ln \left(Q^{2} / \Lambda^{2}\right) \rightarrow \ln \left(Q^{2} / \Lambda^{2}\right)+A$, where $A$ is very small (and negative). However, the NLO contribution to the kernel is renormalization scheme dependent, and this result is in $\overline{\mathrm{MS}}$ scheme. By transferring to schemes that the authors reasonably argue are more suited to gluon dominated processes, i.e., the MOM [57] or $Y \rightarrow g g g$ [24] schemes, the scale change at $\gamma=\frac{1}{2}$ becomes $\widetilde{Q}^{2} \sim 120 Q^{2}$, and the intercept becomes $\sim \lambda\left(\widetilde{Q}^{2}\right)\left(1-4 \alpha_{s}\left(\widetilde{Q}^{2}\right)\right)$. Hence, the large increase in scale and significant reduction in the NLO coefficient leads to a sensible NLO intercept of $\sim 0.15$ which is not too sensitive to $Q^{2}$. I believe the eigenvalue of the kernel is an inappropriate place to make the scale choice since, as soon as one introduces the running coupling into the BFKL equation, the whole structure changes. The $Q^{2}$-dependent eigenvalue is no longer a real eigenvalue, as it is at strictly LO, and it no longer has a direct physical interpretation. This is identical to the statement that the argument of the exponent in Eq. (6.8) does not in fact truly represent the full evolution of any physical quantity, is by no means a true anomalous dimension, and should not be used for setting the scale. In essence the choice in [55] misses the most important results generated by solving the BFKL equation with running coupling and looking at physical quantities. This is easily seen by the fact that in any renormalization scheme the change in scale using the method in [55] is always of the form $\ln \left(\widetilde{Q}^{2} / \Lambda^{2}\right)$ $=\ln \left(Q^{2} / \Lambda^{2}\right)+A_{r s}$, where $A_{r s}$ is a constant depending on the scheme. Using the BLM method for physical quantities, as in this paper, always results in $\ln \left(\widetilde{Q}^{2} / \Lambda^{2}\right)=\ln \left(Q^{2} / \Lambda^{2}\right)+B_{r s, i}$ $+3.63\left(\bar{\alpha}_{s}\left(Q^{2}\right) \xi\right)^{1 / 2}$, where $B_{r s, i}$ depends on renormalization scheme and process. Clearly the $\xi$-dependent term is the dominant one at small enough $x$ and contains the most important physics contributing to the scale fixing. Note that this contribution is also scheme-independent and the same at NLO as at LO, and that the choice of renormalization scheme only leads to subleading contributions to the scale at small $x$. Nevertheless, the type of renormalization scheme considered in [55] leads to a value of $B_{r s, i}$ that is rather large. This implies that the details of calculations of structure functions in the current experimental range may be sensitive to the renormalization scheme chosen. However, when doing a full analysis one should use the same scheme for all physical splitting functions, which will be influenced by both gluon and quark dominated processes. There are also further changes to the scale due to the running coupling effects at $\mathcal{O}\left(\alpha_{s}^{2}\right)$, which will be scheme dependent, and potentially of similar importance to the differences in $B_{r s, i}$ at the relatively high $x$ values where it is relevant. A full understanding of the relevance of renormalization scheme changes needs to take these into account carefully.

Hence, to summarize, I believe that the method of solving for physical quantities using the BFKL equation with running coupling and full NLO contributions presented in this paper is the best way to proceed for the analysis of deep inelastic scattering at small $x$. Certainly, the conclusion that the running coupling serious alters our picture of BFKL physics, destroying predictivity for the input and maintaining it, but smoothing out the powerlike behavior for the calculable evolution, seems to be incontrovertible. More controversial is the proposal that the true physics may be well described by a coupling which falls as $x$ falls like $\ln (1 / x)^{-1 / 2}$. This is strongly supported by current finite order expansions, the universality between deep inelastic scattering (DIS) processes and different orders, the diffusion picture, and the general features that the full solution must exhibit. However, it may well be possible to validate this more strongly, or invalidate it. Also, the discussion in this paper has very firmly used the assumption that the lower end of the gluon ladder is fixed at some low scale, as is appropriate for deep inelastic scattering. Further investigation is required in 
order to consider different types of process, although I imagine that the qualitative results will be the same. Overall normalization will be infrared renormalon contaminated, since even if there are no small scales in the problem the diffusion into the infrared will eventually be important for small enough $x$, while evolution will be calculable but not truly powerlike. If the general results of this paper are correct, perturbative calculations at small $x$ will be very reliable and convergent. They would also explain why perturbation theory appears, at least qualitatively, to be working at very low scales at small $x$, but also implies that the standard NLO in $\alpha_{s}\left(Q^{2}\right)$ approach is not really quantitatively correct at small $x$. More phenomenological work, including calculation of currently unknown coefficient functions as power series in $\alpha_{s} \ln (1 / x)$, would then be important in order to produce truly precise calculations for small $x$ physics.

\section{ACKNOWLEDGMENTS}

I would like to thank Dick Roberts for continual help during the period of this work and for the use of the MartinRoberts-Stirling (MRS) fit program. I would also like to thank Stefano Catani, Jeff Forshaw, Alex Kovner, Martin McDermott, Graham Ross, Davis Soper, George Sterman, and Mark Wusthoff for useful discussions.
[1] L. N. Lipatov Yad. Fiz. 23, 642 (1976) [Sov. J. Nucl. Phys. 23, 338 (1976)]; E. A. Kuraev, L. N. Lipatov, and V. S. Fadin, Zh. Eksp. Teor. Fiz. 72, 377 (1977) [Sov. Phys. JETP 45, 199 (1977)]; Ya. Balitskii and L. N. Lipatov, Yad. Fiz. 28, 12 (1978) [Sov. J. Nucl. Phys. 28, 5 (1978)].

[2] V. S. Fadin and L. N. Lipatov, Phys. Lett. B 429, 127 (1998), and references therein.

[3] G. Camici and M. Ciafaloni, Phys. Lett. B 430, 349 (1998), and references therein.

[4] J. Blümlein, V. Ravindran, W. L. van Neerven, and A. Vogt, in Proceedings of DIS 98, Brussels, 1998, p. 211, hep-ph/9806368.

[5] R. D. Ball and S. Forte, in Proceedings of DIS 98, Brussels, 1998, p. 770, hep-ph/9805315.

[6] D. A. Ross, Phys. Lett. B 431, 161 (1998).

[7] Yu. V. Kovchegov and A. H. Mueller, Phys. Lett. B 439, 423 (1998).

[8] E. M. Levin, Tel Aviv University, Report No. TAUP 2501-98, hep-ph/9806228.

[9] N. Armesto, J. Bartels, and M. A. Braun, Phys. Lett. B 442, 459 (1998).

[10] G. Salam, J. High Energy Phys. 07, 19 (1998).

[11] M. Ciafaloni and D. Colferai, Phys. Lett. B 452, 372 (1999).

[12] L. V. Gribov, E. M. Levin, and M. G. Ryskin, Phys. Rep. 100, 1 (1983).

[13] L. N. Lipatov, Zh. Eksp. Teor. Fiz. [Sov. Phys. JETP 63, 904 (1986)].

[14] J. Kwiecinski, Z. Phys. C 29, 561 (1985).

[15] J. C. Collins and J. Kwiecinski, Nucl. Phys. B316, 307 (1989).

[16] R. E. Hancock and D. A. Ross, Nucl. Phys. B383, 575 (1992); B394, 200 (1993).

[17] N. N. Nikolaev and B. G. Zakharov, Phys. Lett. B 327, 157 (1994).

[18] E. M. Levin, Nucl. Phys. B453, 303 (1995).

[19] B. Andersson, G. Gustafson, and H. Kharraziha, Phys. Rev. D 57, 5543 (1998).

[20] L. P. A. Haakman, O. V. Kancheli, and J. H. Koch, Phys. Lett. B 391, 157 (1997); Nucl. Phys. B518, 275 (1998).

[21] G. Camici and M. Ciafaloni, Phys. Lett. B 395, 118 (1997).

[22] J. Bartels and H. Lotter, Phys. Lett. B 309, 400 (1993); J. Bartels, H. Lotter, and M. Vogt, ibid. 373, 215 (1996).
[23] A. H. Mueller, Phys. Lett. B 396, 251 (1997).

[24] S. J. Brodsky, G. P. Lepage, and P. B. Mackenzie, Phys. Rev. D 28, 228 (1983).

[25] S. Catani, M. Ciafaloni, and F. Hautmann, Phys. Lett. B 242, 97 (1990); Nucl. Phys. B366, 135 (1991); Phys. Lett. B 307, 147 (1993).

[26] J. C. Collins and R. K. Ellis, Nucl. Phys. B360, 3 (1991).

[27] J. R. Forshaw, G. P. Salam, and R. S. Thorne, in Proceedings of 3rd UK Phenomenology Workshop on HERA Physics, Durham, 1998, hep-ph/9812304.

[28] T. Jaroszewicz, Phys. Lett. 116B, 291 (1982).

[29] J. R. Forshaw, P. N. Harriman, and P. N. Sutton, Nucl. Phys. B416, 739 (1994).

[30] J. C. Collins and P. V. Landshoff, Phys. Lett. B 276, 196 (1992).

[31] M. F. McDermott, J. R. Forshaw, and G. G. Ross, Phys. Lett. B 349, 189 (1995); M. F. McDermott and J. R. Forshaw, Nucl. Phys. B484, 248 (1997).

[32] J. R. Forshaw and P. N. Harriman, Phys. Rev. D 46, 3778 (1992).

[33] A. D. Martin and J. Kwiecinski, Phys. Lett. B 353, 123 (1995).

[34] V. I. Zakharov, Nucl. Phys. B385, 452 (1992); M. Beneke, ibid. B405, 424 (1993).

[35] G. Camici and M. Ciafaloni, Phys. Lett. B 386, 341 (1996).

[36] S. J. Brodsky and H. J. Lu, Phys. Rev. D 51, 3652 (1995).

[37] S. Catani, in Proceedings of DIS 96, Rome, 1996, p. 165, hep-ph/9608310; S. Catani, Z. Phys. C 75, 665 (1997).

[38] S. Catani and F. Hautmann, Phys. Lett. B 315, 157 (1993); Nucl. Phys. B427, 475 (1994).

[39] J. Blümlein and A. Vogt, Phys. Rev. D 57, 1 (1998); 58, 014020 (1998).

[40] H1 Collaboration, S. Aid et al., Nucl. Phys. B470, 3 (1996); H1 Collaboration, C. Adloff et al., ibid. B497, 3 (1997).

[41] ZEUS Collaboration, M. Derrick et al., Z. Phys. C 69, 607 (1996); ZEUS Collaboration, M. Derrick et al., ibid. 72, 399 (1996).

[42] R. S. Thorne, Phys. Lett. B 392, 463 (1997); Nucl. Phys. B512, 323 (1998)

[43] W. K. Wong, Phys. Rev. D 54, 1094 (1996).

[44] R. S. Thorne (in preparation). 
[45] R. S. Thorne, in Proceedings of DIS 98, Brussels, 1998, p. 207, hep-ph/9805299.

[46] R. S. Thorne (in preparation).

[47] A. D. Martin, R. G. Roberts, W. J. Stirling, and R. S. Thorne, Eur. Phys. J. C 4, 463 (1998).

[48] R. G. Roberts, hep-ph/9904317.

[49] A. Caldwell, talk at the DESY Theory Workshop on "Recent Developments in QCD,'” 1997.

[50] ZEUS Collaboration, J. Breitweg et al., Eur. Phys. J. C 7, 609 (1999).

[51] M. Glück, E. Reya, and A. Vogt, Z. Phys. C 67, 433 (1995);
Eur. Phys. J. C 5, 461 (1998).

[52] N. N. Nikolaev and V. R. Zoller, JETP Lett. 69, 103 (1999).

[53] Yu. L. Dokshitzer and B. R. Webber, Phys. Lett. B 352, 451 (1995); M. Dasgupta and B. R. Webber, ibid. 382, 96 (1996).

[54] C. Bontus, talk at Durham HERA workshop, 1998.

[55] S. J. Brodsky, V. S. Fadin, V. T. Kim, L. N. Lipatov, and G. B. Pivovarov, SLAC-PUB-8037, IITAP-98-010, hep-ph/9901229.

[56] G. Camici and M. Ciafaloni, Nucl. Phys. B496, 305 (1997).

[57] W. Celmaster and R. J. Gonsalves, Phys. Rev. D 20, 1420 (1979); Phys. Rev. Lett. 42, 1435 (1979); P. Pascual and R. Tarrach, Nucl. Phys. B174, 123 (1980); B181, 546(E) (1981). 